\title{
Two isoforms of the essential $C$. elegans Argonaute CSR-1 differentially regulate sperm and oocyte fertility
}

\begin{abstract}
Amanda G. Charlesworth ${ }^{1, \#}$, Uri Seroussi ${ }^{1, \#}$, Nicolas J. Lehrbach ${ }^{2,3, \#, ~ M a t h i a s ~ S . ~ R e n a u d ~}{ }^{1}$, Adam E. Sundby ${ }^{1}$, Ruxandra I. Molnar ${ }^{1}$, Robert X. Lao ${ }^{1}$, Alexandra R. Willis', Jenna R. Woock', Matthew J. Aber ${ }^{2,3}$, Annette J. Diao ${ }^{1}$, Aaron W. Reinke ${ }^{1}$, Gary Ruvkun ${ }^{2,3}$, Julie M. Claycomb ${ }^{1 *}$
\end{abstract}

${ }^{1}$ Department of Molecular Genetics, University of Toronto Toronto, ON M6G2Z7 Canada ${ }^{2}$ Department of Molecular Biology, Massachusetts General Hospital, Boston, MA 02114, USA

${ }^{3}$ Department of Genetics, Harvard Medical School, Boston, MA 02115, USA

"Equal contribution

*Correspondence: julie.claycomb@utoronto.ca 


\section{ABSTRACT}

The C. elegans genome encodes nineteen functional Argonaute proteins that use 22G-RNAs, 26G-RNAs, miRNAs, or piRNAs to regulate target transcripts. Only one Argonaute is essential under normal laboratory conditions: CSR-1. While CSR-1 has been studied widely, nearly all studies have overlooked the fact that the csr-1 locus encodes two isoforms. These isoforms differ by an additional 163 amino acids present in the N-terminus of CSR-1a. Using CRISPR-Cas9 genome editing to introduce GFP::3xFLAG into the long (CSR-1a) and short (CSR-1b) isoforms, we found that CSR-1a is expressed during spermatogenesis and in several somatic tissues, including the intestine. CSR-1b is expressed constitutively in the germline. small RNA sequencing of CSR-1 complexes shows that they interact with partly overlapping sets of 22G-RNAs. Phenotypic analyses reveal that the essential functions of $c s r-1$ described in the literature coincide with CSR-1b, while CSR-1a plays tissue specific functions. During spermatogenesis, CSR-1a integrates into an sRNA regulatory network including ALG3, ALG-4, and WAGO-10 that is necessary for fertility at $25^{\circ} \mathrm{C}$. In the intestine, CSR-1a silences immunity and pathogen-responsive genes, and its loss results in improved survival from the pathogen Pseudomonas aeruginosa. Our findings functionally distinguish the CSR-1 isoforms and highlight the importance of studying each AGO isoform independently.

\section{KEY POINTS}

- The essential AGO CSR-1 encodes two isoforms that vary in expression, target transcripts, and functions.

- CSR-1a and CSR-1b integrate differently into germline small RNA regulatory networks. CSR-1a is required for sperm-mediated fertility under stress, while CSR-1b is essential for oocyte-mediated fertility and embryonic survival.

- CSR-1a is also important in the intestine where it silences immunity genes. Its loss leads to improved survival from the bacterial pathogen Pseudomonas aeruginosa. 


\section{INTRODUCTION}

From the discovery of miRNAs to understanding the molecular mechanisms of RNA interference (RNAi), the nematode $C$. elegans has been a champion of sRNA biology (1). At the core of small RNA (sRNA) mediated gene regulatory pathways are the conserved Argonaute (AGO) proteins, which are guided in a sequence specific manner by sRNAs to impart a variety of gene regulatory outcomes on target transcripts (2). C. elegans possesses a remarkable expanded family of nineteen AGO proteins which function in association with four types of sRNAs (including miRNAs, piRNAs, 22G-RNAs, and 26G-RNAs). These sRNA pathways regulate gene expression to orchestrate normal developmental and differentiation programs, as well as to navigate stressful environmental conditions, such as elevated temperature (3) (Seroussi, in preparation). The scope of sRNA mediated gene regulatory potential in the worm grows more complex when considering that several AGOs, including ALG-1, ALG2, ERGO-1, PPW-1, and CSR-1 are predicted to be expressed as multiple isoforms, which have not been characterized.

The AGO CSR-1 (Chromosome Segregation and RNAi Deficient) is one of the most intensively studied AGOs in $C$. elegans, owing in part to the fact that it is the only solely essential ago, and to its potentially conflicting and varied roles in gene regulation throughout development $(3,4)$. The csr-1 locus encodes two isoforms, CSR-1a and CSR-1b, that vary only in their N-terminus by 163 amino acids (4). Within this extended N-terminal region of CSR-1a are fifteen arginine/glycine RGG/RG motifs, many of which are arranged into di- or tri-RGG/RG motifs (5). In AGOs of other species, including Drosophila Piwi, RGG/RG motifs are the sites of arginine di-methylation, which is required for the recruitment of additional factors, such as Tudor proteins, to ensure functionality of the AGO/sRNA complex (called the RISC; RNA Induced Silencing Complex) (6-8). These motifs are also typical of RNA binding proteins, and have been identified in association with phase separated RNA rich cytoplasmic granules, such as germ granules (9). The role and importance of the RGG/RG motifs in CSR-1a have not been determined, and CSR-1 has been widely studied as a single protein, thus any differential functions of CSR-1 isoforms during development are currently unknown. 
Previous studies indicated that CSR-1 is expressed in both the germline and soma in hermaphrodites $(4,10)$. Immunostaining with antibodies that recognize both CSR-1 isoforms showed that CSR-1 is present in the hermaphrodite germline throughout all stages of germline development, from the two-cell embryo to spermatogenesis and oogenesis (4). Within the germline, CSR-1 is found in the cytoplasm, and is enriched in phase separated germ granules known as P granules (4). In oocytes and during early embryonic cell divisions, CSR-1 is present within the nucleus and associates with condensed chromosomes upon nuclear envelope breakdown (4). In the soma, CSR-1 was observed within intestinal cells, where it was detected in both the cytoplasm and nucleus $(4,10)$. The germline expression pattern of CSR-1 highlights its role in fertility, as homozygous null mutants that eliminate both csr-1 isoforms are nearly sterile, and the few embryos produced display chromosome segregation defects during early embryonic divisions that result in lethality $(3,4,11)$. However, which CSR-1 isoform is expressed in which tissues, and which isoform is necessary for fertility are unexplored.

While most AGOs are thought of as gene silencers, CSR-1 has been shown to play both positive (licensing) and negative (silencing) gene regulatory roles (11-13). CSR-1 associates with a type of endogenous sRNA known as 22G-RNAs that target nearly 5000 germline-expressed protein coding genes (about $25 \%$ of the genome) $(4,14)$. CSR-1-associated $22 \mathrm{G}-\mathrm{RNAs}$ are produced by the RNA dependent RNA polymerase (RdRP), EGO-1, one of four RdRPs present in the worm $(4,15)$. Microarray and GRO-seq studies demonstrated that loss of csr-1 does not result in the global upregulation of the majority of target transcripts $(4,11,14,16)$. Instead, most CSR-1 target transcripts decrease in expression levels in csr-1 mutants, pointing to its role in the protection or licensing of target transcripts, rather than their destruction or silencing.

There are several ways in which CSR-1 has been implicated in promoting the expression of its target transcripts. First, CSR-1 has been shown to promote the expression of histones. In this capacity, CSR-1 is thought to act as an endonuclease or "Slicer," where it cleaves the 3' stem loop of histone RNA transcripts enabling them to be translated. Loss of $c s r-1$ leads to decreased levels of histone transcripts and proteins (10). Second, CSR-1 licenses the expression of germline GFP transgenes in opposition to the silencing effects of the piRNA pathway, in a process related to paramutation, termed 
RNA activation (RNAa) $(17,18)$. Third, CSR-1 promotes the formation of euchromatin at its numerous target gene loci throughout the genome, and loss of csr-1 leads to aberrant accumulation of heterochromatic histone modifications and decreased transcription at target gene loci $(16,19)$.

CSR-1 is one of only a few AGOs which possess a predicted Slicer motif, DEDH, within its PIWI domain, rendering it capable of endonucleolytic cleavage of target transcripts. CSR-1 exhibits Slicer activity in vitro (20), and mutation of the Slicer residues leads to embryonic lethality, although at a later developmental stage than null mutants $(11,13)$. Slicer activity is required for several context-specific functions, including fine tuning kinetochore and cell cycle gene expression during embryogenesis and clearing maternal mRNAs at the oocyte to embryo transition, RNAi, processing histone mRNA transcripts, and stimulating the production of 22G-RNAs $(4,10,11,13,20,21)$.

While most studies of CSR-1 have focused on the oogenic gonad and embryos, CSR-1 has also been implicated in spermatogenesis. Spermatogenesis occurs in the L4 larval stage of development, prior to the onset of oogenesis (22). In spermatogenesis, the AGOs ALG-3 and -4 regulate a set of spermatogenesis transcripts both positively and negatively via endogenous sRNAs called 26G-RNAs $(14,23)$. 26G-RNAs are produced by the RdRP RRF-3, which synthesizes dsRNA that is processed by the endonuclease Dicer and the phosphatase PIR-1 (24). The targeting of transcripts by 26G-RNAs leads to the amplification of 22G-RNAs by the RdRPs EGO-1 and RRF-1, and the subsequent targeting of the same transcripts by different 22G-RNA/AGO RISCs (23, 25-27). alg-3; alg-4 double mutants display a progressive loss of male and hermaphrodite fertility due to spermatogenesis defects $(14,23)$. Curiously, while loss of $c s r-1$ in hermaphrodites leads to sterility in one generation $(3,4)$, loss of csr-1 in males leads progressive loss of fertility similar to that of alg-3, alg-4 double mutants (14), suggesting a distinct role for CSR-1 in spermatogenesis.

The characterization of CSR-1 associated 22G-RNAs in males showed that CSR-1 targets a set of protein coding genes in common with CSR-1 targets in hermaphrodites undergoing oogenesis, plus an additional set of genes. Furthermore, a subset of the genes targeted by CSR-1 during spermatogenesis overlap with genes targeted by ALG-3 and ALG-4. Because 26G-RNAs associated with ALG-3 and -4 are thought to lead to the production of 22G-RNAs, this places CSR-1 downstream 
of ALG-3 and -4 during spermatogenesis (14). Consistent with its role in oogenesis, CSR-1 positively regulates its spermatogenic targets at the level of transcription during spermatogenesis (14), and depletion of $c s r-1$ by RNAi led to the upregulation of spermatogenesis genes in oogenesis (28). Why CSR-1 regulates the same subsets of genes in the spermatogenic and oogenic gonad, and which CSR1 isoform(s) affect male fertility remain to be determined. Finally, although CSR-1 has been detected in the germline throughout development, transcriptomic analyses of isolated male and female $C$. elegans gonads showed that the csr-1a mRNA is abundant in the spermatogenic gonad, but is not highly expressed during oogenesis. These data point to a potential role for CSR-1a specifically during spermatogenesis that has not been explored (29).

Here we show that the two isoforms of CSR-1 have distinct expression profiles throughout development. The long isoform, CSR-1a, is expressed in the germline only during spermatogenesis, while the short isoform, CSR-1b, is expressed constitutively in the germline throughout all stages of development. During spermatogenesis, CSR-1a and CSR-1b integrate into a larger sRNA network to regulate many facets of spermatogenesis. While CSR-1b, in conjunction with WAGO-4, associates with 22G-RNAs to regulate germline constitutive (i.e. expressed both during spermatogenesis and oogenesis) and oogenic transcripts (29), CSR-1a associates with 22G-RNAs to target a set of spermatogenesis-enriched transcripts (29). CSR-1a and another uncharacterized AGO, WAGO-10, integrate into the ALG-3 and -4 26G-RNA pathway to enable male fertility during temperature stress. CSR-1b also plays a role in regulating spermatogenesis genes, but is essential for hermaphrodite fertility and embryonic viability. CSR-1a is also expressed in several somatic tissues, including the intestine, where it regulates immunity and pathogen response genes. Remarkably, loss of csr-1a renders worms more resistant to death by the bacterial pathogen Pseudomonas aeruginosa.

Collectively, our data demonstrate that different AGO isoforms can display distinct expression profiles, and are capable of different functions, highlighting the importance of studying each AGO isoform as a separate entity.

\section{MATERIALS AND METHODS}




\section{C. elegans Genetics and Culture}

C. elegans strains were derived from the wild type Bristol N2 strain and cultured according to standard growth conditions (30) on Nematode Growth Medium (NGM) plates inoculated with E. coli OP50 at $20^{\circ} \mathrm{C}$, unless otherwise stated. Synchronised worms were reared at $20^{\circ} \mathrm{C}$ and harvested as follows: L1s at 8 hours after plating, L2s at 26 hours, L3s at 34.5 hours, L4s at 48 hours, young adults at 60 hours and gravid adults at 72 hours. Embryos were collected from gravid adult worms by standard bleaching 72 hours after plating.

\section{Strains used in this study}

\begin{tabular}{|c|c|c|}
\hline Experimental Models: Organisms/Strains & Source & $\begin{array}{l}\text { Strain } \\
\text { Name }\end{array}$ \\
\hline Wild-type C. elegans, Bristol Strain & CGC & N2 \\
\hline $\begin{array}{l}\text { csr-1(tor67[gfp::3xflag::csr-1]) IV } \\
\text { [made with CRISPR with SEC] }\end{array}$ & Ouyang et al., 2019 & JMC101 \\
\hline $\begin{array}{l}\text { csr-1(tor160[csr-1 exon1:::gfp:::3xflag IV:7957568]) IV } \\
\text { [made with CRISPR with SEC] }\end{array}$ & This study & JMC151 \\
\hline $\begin{array}{l}\text { csr-1(tor67[csr-1 exon2::gfp::3xflag IV:7958598], csr-1(mg660[G120*])) IV } \\
\text { [made with Co-CRISPR into JMC101] }\end{array}$ & This study & JMC164 \\
\hline csr-1(tm892) IV/nT1 [unc-?(n754) let-?] (IV;V) & $\begin{array}{l}\text { Yigit et al., 2006; } \\
\text { Claycomb et al., } \\
2009\end{array}$ & WM182 \\
\hline unc-119(ed3) III; mgTi13[rpl-28::gfp:::csr-1b]; csr-1(tm892) & This study & JMC160 \\
\hline unc-119(ed3) III; mgTi11[rpl-28::csr-1a exon1::gfp]; csr-1(tm892) & This study & JMC161 \\
\hline
\end{tabular}




\begin{tabular}{|c|c|c|}
\hline mgls75[rpn-2::gfp] IV; mgTi14[pbs-5::mcherry] & This study & GR3035 \\
\hline $\begin{array}{l}\text { unc-119(ed3) III; mgTi11[rpl-28::csr-1a exon1::gfp:::csr-1] } \\
\text { [made with MiniMos] }\end{array}$ & This study & GR3037 \\
\hline $\operatorname{csr}-1$ (mg657[G91R]) IV & This study & GR3039 \\
\hline $\begin{array}{l}\text { unc-119(ed3) III; mgTi13[rpl-28::gfp::csr-1b] } \\
\text { [made with MiniMos] }\end{array}$ & This study & GR3041 \\
\hline $\operatorname{csr}-1\left(m g 660\left[G 120^{*}\right]\right) I V$ & This study & GR3042 \\
\hline csr-1(mg661[G120*]) mgls75[rpn-2::gfp] IV; mgTi14[pbs-5::mcherry] & This study & GR3043 \\
\hline wago-10(tor133) $V$ & This study & JMC192 \\
\hline alg-4(tm1184) III & Yigit et al., 2006 & \\
\hline alg-3(tm1155) IV & Yigit et al., 2006 & \\
\hline $\begin{array}{l}\text { alg-4(tm1184) III; csr-1(tor67[csr-1 exon2::gfp::3xflag IV:7958598], csr- } \\
\text { 1(mg660[G120*])) IV, alg-3(tm1155) IV; wago-10(tor133) V }\end{array}$ & This study & JMC245 \\
\hline alg-3(tor141[gfp::3xflag::alg-3]) IV & This study & JMC205 \\
\hline alg-4(tor143[gfp::3xflag::alg-4]) III & This study & JMC207 \\
\hline wago-10(tor127[gfp::3xflag::wago-10]) V & This study & JMC233 \\
\hline wago-10(tor162[3xflag::wago-10]) V & This study & JMC248 \\
\hline wago-4(tor117[gfp::3xflag::wago-4]) II & Lev et al., 2019 & JMC223 \\
\hline pha-1(e2123) & $\begin{array}{l}\text { Schnabel and } \\
\text { Schnabel, } 1990\end{array}$ & GE24 \\
\hline
\end{tabular}




\begin{tabular}{|c|c|c|}
\hline znfx-1(gg634[HA::tagRFP::znfx-1]) II. & Wan et al., 2018 & YY1446 \\
\hline pgl-1(gg640[pgl-1::flag::mCardinal]) IV & Wan et al., 2018 & YY1492 \\
\hline pgl-1(zuls252[Pnmy-21::PGL-1::mRFP; unc119(+)] unc119(ed3)) III; & Wolke et al., 2007 & JJ2213 \\
\hline wago-4(tor117[gfp::3xflag::wago-4]) II, znfx-1(gg634[HA::tagRFP::znfx-1]) II & This study & JMC255 \\
\hline $\begin{array}{l}\text { znfx-1(gg634[HA::tagRFP::znfx-1]) II; csr-1(tor160[csr-1 exon1::gfp::3xflag } \\
\text { IV:7957568]) IV }\end{array}$ & This study & JMC259 \\
\hline $\begin{array}{l}\text { znfx-1(gg634[HA::tagRFP::znfx-1]) II; csr-1(tor67[csr-1 exon2::gfp::3xflag } \\
\text { IV:7958598], csr-1(mg660[G120*])) IV }\end{array}$ & This study & JMC260 \\
\hline $\begin{array}{l}\text { pgl-1(zuls252[Pnmy-21::PGL-1::mRFP; unc119(+)] unc119(ed3)) III; csr- } \\
\text { 1(tor160[csr-1 exon1::gfp::3xflag IV:7957568]) IV }\end{array}$ & This study & JMC277 \\
\hline $\begin{array}{l}\text { pgl-1(zuls252[Pnmy-21::PGL-1::mRFP; unc119(+)] unc119(ed3)) III; csr- } \\
\text { 1(tor67[csr-1 exon2:::gfp::3xflag IV:7958598], csr-1(mg660[G120*])) IV }\end{array}$ & This study & JMC278 \\
\hline $\begin{array}{l}\text { znfx-1(gg634[HA::tagRFP::znfx-1]) II, wago-4(tm1019) II; csr-1(tor160[csr-1 } \\
\text { exon1::gfp::3xflag IV:7957568]) IV }\end{array}$ & This study & JMC279 \\
\hline $\begin{array}{l}\text { znfx-1(gg634[HA::tagRFP::znfx-1]) II, wago-4(tm1019) II; csr-1(tor67[csr-1 } \\
\text { exon2::gfp::3xflag IV:7958598], csr-1(mg660[G120*])) IV }\end{array}$ & This study & JMC276 \\
\hline $\begin{array}{l}\text { znfx-1(gg634[HA::tagRFP::znfx-1]) II, wago-4(tor117[gfp::3xflag::wago-4]) II; } \\
\text { csr-1(mg660[G120*]) IV }\end{array}$ & This study & JMC275 \\
\hline $\begin{array}{l}\text { wago-4(tor117[gfp::3xflag:::wago-4]) II; pgl-1(zuls252[Pnmy-21::PGL-1::mRFP; } \\
\text { unc119(+)] unc119(ed3)) III; csr-1(mg660[G120*]) IV }\end{array}$ & This study & JMC283 \\
\hline $\begin{array}{l}\text { wago-4(tm1019) II; pgl-1(zuls252[Pnmy-21::PGL-1::mRFP; unc119(+)] } \\
\text { unc119(ed3)) III; csr-1(tor160[csr-1 exon1::gfp::3xflag IV:7957568]) IV }\end{array}$ & This study & JMC284 \\
\hline $\begin{array}{l}\text { wago-4(tm1019) II; pgl-1(zuls252[Pnmy-21::PGL-1::mRFP; unc119(+)] } \\
\text { unc119(ed3)) III; csr-1(tor67[csr-1 exon2:::gfp::3xflag IV:7958598], csr- } \\
\text { 1(mg660[G120*])) IV }\end{array}$ & This study & JMC285 \\
\hline
\end{tabular}




\section{Strain construction and validation}

Strains were constructed using the following methods. Genotypes are presented in the Strains Table.

\section{Co-CRISPR:}

Strains JMC164, GR3042, GR3043 were constructed from JMC101, GE24 [pha-1(e2123) III] (31), and GR3042 respectively, using the Co-CRISPR technique. All sgRNA sequences were inserted into pDD162 by New England Biolabs Q5 site-directed mutagenesis as described (32). Repair template single-stranded DNA oligonucleotides were designed as described (33, 34). Injections were performed using editing of $d p y-10$ (to generate cn64 rollers) as a phenotypic co-CRISPR marker $(34,35)$. Injection mixes contained $60 \mathrm{ng} / \mu \mathrm{L}$ each of the co-CRISPR and gene of interest sgRNA/Cas 9 construct, and $50 \mathrm{ng} / \mu \mathrm{L}$ each of the co-CRISPR and gene of interest repair template oligonucleotides. To generate the wago-10(tor133) allele, which deletes the region between nucleotides 695-2394 (1699nt deletion), multiple sgRNAs spanning the wago-10 genomic region were designed and injected along with the $d p y$ 10 co-CRISPR plasmid as described above. Insertion/deletion events were screened by PCR. sgRNA and homologous repair template sequences are listed in Supplemental Table S3. All CRISPR edits were confirmed by sequencing.

CRISPR/Cas9 homologous with self-excising cassette:

CRISPR was performed as described (36-38). The repair templates were amplified using primers listed in the Supplemental Table S3 and introduced into the vector pDD282 (Addgene \#66823) by isothermal assembly (39). Plasmids expressing guide RNAs were generated with the NEBuilder kit (New England BioLabs) using pDD162 (Addgene \#47549) and the primers listed in the Supplemental table S3. 
miniMos transgenesis:

Cloning was performed by isothermal/Gibson assembly (39). All plasmids used for transgenesis are listed in Supplemental Table S3, and were assembled using pNL43 (40). MiniMos injections were performed as described in (41). To make the strain GR3037, exon 1 of csr-1a (a 474 bp fragment beginning at the start codon of csr-1a) was placed downstream of the rpl-28 promoter (605 bp immediately upstream of the rpl-28 start codon) and fused in-frame at the $5^{\prime}$ end of GFP. The $3^{\prime}$ end of the GFP coding sequence was fused in-frame with a DNA fragment containing the remaining exons of csr-1a and the csr-1 3'UTR (3829 bp beginning at the first codon of exon 2 of csr-1a). This construct (assembled in pNL201 and used to generate mgTi11 and mgTi12) drives the ubiquitous expression of CSR-1a with an internal GFP tag inserted after K158. To make strain GR3041, the DNA fragment containing GFP fused in-frame to csr-1a exons 2 through the csr-1 3'UTR (4697 bp, from pNL201) was placed downstream of the rpl-28 promoter (605 bp immediately upstream of the rpl-28 start codon). This construct (assembled in pNL213 and used to generate mgTi13) drives the ubiquitous expression of CSR-1b fused to GFP at its N-terminus via a short linker with the sequence 'GLNSD'.

Generation of the rpn-2::gfp transgene:

The integrated mgls75[rpn-2::gfp::unc-54] transgene was generated from sEx10255 (42). EMS mutagenesis was used to induce integration of the extrachromosomal array onto chromosome IV.

\section{Mutagenesis, screening and genome sequencing}

Mutagenesis was performed by treatment of L4 animals in $47 \mathrm{mM}$ EMS for 4 hours at $20^{\circ} \mathrm{C}$. Genomic DNA was prepared using the Gentra Puregene Tissue kit (Qiagen) according to the manufacturer's instructions. Genomic DNA libraries were prepared using the NEBNext genomic DNA library construction kit (New England Biololabs), and sequenced on an Illumina Hiseq. Deep sequencing reads were analyzed using Cloudmap (43). 


\section{Microscopy}

Live fluorescence microscopy of CSR-1::GFP

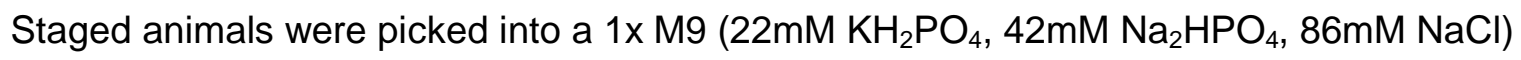
droplet with $2 \mu \mathrm{l}$ of $2 \mathrm{mM}$ levamisole on standard microscopy slides with $2 \%$ agarose pads. Imaging was performed on a Nikon TiE inverted microscope and Nikon C2 confocal system with a PL Apochromat Lambda 60x Oil Immersion objective. Images were acquired using NIS Elements AR software. For time course images, larvae and embryo images were taken every 60 seconds. All images were acquired with the same settings and exported via NIS-Elements AR Software. Any post-acquisition adjustments made to images were applied equally between control and experimental sets using Fiji (44) and Nikon NIS viewer.

Imaging released sperm

Males were moved to plates without hermaphrodites 1-2 days prior to imaging to maximize sperm retention. Males were soaked in $2 \mathrm{ml}$ of $1 \mathrm{X}$ Sperm Buffer $(50 \mathrm{mM}$ Hepes, $25 \mathrm{mM} \mathrm{KCl,} 35 \mathrm{mM} \mathrm{NaCl}$, $1 \mathrm{mM} \mathrm{MgSO}_{4}, 5 \mathrm{mM} \mathrm{CaCl}_{2}, 10 \mathrm{mM}$ Dextrose/D-Glucose, $\mathrm{pH} 7.8$, filter sterilized) with $0.5 \mu \mathrm{l}$ of $5 \mu \mathrm{g} / \mathrm{ml}$ DAPI for 5 minutes in a crystallization dish, then transferred to poly-L-lysine coated slides. $2 \mathrm{mM}$ levamisole was added, and worm tails were cut with a 17 gauge needle to release sperm/germline. Samples were imaged immediately using Sequential Scan Mode on a Leica DMi8 TCS SP8 confocal microscope using a 63x/1.40 HC PL APO CS2 oil objective.

\section{CSR-1 zygotic expression assay}

Male worms homozygous for GFP::3XFLAG::CSR-1 were crossed to N2 hermaphrodites. After 24 hours, hermaphrodites were collected and dissected to obtain early embryos. Later staged embryos and L1 larvae were obtained by washing plates with 1X M9. Embryos and larvae were transferred to slides with $2 \%$ agarose pads. $2 \mu$ of $2 \mathrm{mM}$ levamisole was added to immobilize larvae. Samples were imaged at 60x on a Nikon TiE inverted microscope and Nikon C2 confocal system utilizing the NIS-

\section{Elements AR Software.}




\section{Imaging and quantification of germ granules}

Worms were washed from plates in M9 and immobilized on positively charged glass slides with M9 and 10 $\mathrm{MM}$ levamisole. Germlines were dissected with a 17 gauge needle and then imaged immediately on a Leica DMi8 TCS SP8 confocal microscope using a 63x/1.40 HC PL APO CS2 oil objective. All images were collected using the same acquisition settings. Colocalization of proteins was calculated with the ImageJ plugin JaCoP (45). One germline from each of six different animals was imaged per strain and developmental time point. Regions of interest $(\mathrm{ROI})$ were generated using the 3D objects counter plugin in ImageJ (46) by adjusting the threshold until only germ granule pixels are detected. Mander's co-localization coefficients are calculated using JaCoP (45). To count the number of germ granules per nucleus, images were split into separate channels using ImageJ, and the "Find Maxima" function was used to identify granules around each nucleus in each channel. Z-slices corresponding to the center of the nucleus were counted from 20 nuclei per germline in five germlines per genotype (100 nuclei total). To analyze any differences in the number of granules per nucleus among strains, mixed-effects ANOVA was used, as calculated by 'Imer()' in R (version 3.6.3). Hypothesis testing was performed with the 'car' package (47) using Type II sums of squares. Residual data points were normally distributed.

Imaging and analysis of rpn-2::gfp transgenic worms

Bright field and GFP fluorescence images showing rpn-2::GFP expression were collected using a Zeiss AxioZoom V16, equipped with a Hammamatsu Orca flash 4.0 digital camera, using Axiovision software. Images for all strains were collected using the same acquisition settings and then processed identically using ImageJ (46). To quantify rpn-2::GFP expression, the maximum pixel intensity within the intestine of each animal was measured using ImageJ. Values were then normalized such that wild type expression was set to one. 
Gonads and embryos were excised in 1xPBS with 2mM levamisole on poly-L-lysine coated slides from adult hermaphrodites grown at $20^{\circ} \mathrm{C}$. Slides were frozen on dry-ice for at least 10 minutes or stored at $-80^{\circ} \mathrm{C}$ for up to two weeks. Fixation and incubation with primary and secondary antibodies were performed as described by (4). DNA was stained with $1 \mu \mathrm{g} / \mathrm{ml} \mathrm{DAPI}$ in $1 \mathrm{xPBS}$. Imaging was performed on a Nikon TiE inverted microscope and Nikon C2 confocal system with a PL Apochromat Lambda 60x Oil Immersion objective and a Leica DMi8 TCS SP8 using a 63x/1.40 HC PL APO CS2 oil objective. Images were processed using ImageJ software (46).

\section{DAPI staining and imaging L4 germlines}

Wild-type and mutant worms were shifted from $20^{\circ} \mathrm{C}$ to $25^{\circ} \mathrm{C} 24$ hours before imaging. L4-stage worms were methanol fixed and DNA was stained with $1 \mu \mathrm{g} / \mathrm{ml}$ DAPI in 1xPBS. Imaging was performed on a Nikon TiE inverted microscope and Nikon C2 confocal system with a PL Apochromat Lambda 60x Oil Immersion objective. Images were acquired using NIS Elements AR software and maximum intensity projections were generated with ImageJ software (46).

\section{Brood size assays}

All brood size assays were conducted on $\mathrm{N} 2$, tagged strains and mutants reared at $20^{\circ} \mathrm{C}$ and $25^{\circ} \mathrm{C}$. Strains were propagated at $20^{\circ} \mathrm{C}$ prior to the assay. $10-20 \mathrm{~L} 4$ individuals ( 1 worm/plate) were placed at the temperature the assay was performed. Every 24 hours the adult was transferred to a fresh plate and embryos were counted. The number of larvae was scored the following day. This continued until the adult hermaphrodite was no longer laying eggs. The total number of embryos laid by a single hermaphrodite over the course of the egg-laying period was determined (total brood size), as well as the total number of larvae that hatched (viable brood size). Data were plotted using GraphPad Prism or the ggplot2 package in R (48). Statistical analyses in GraphPad Prism used unpaired $t$-tests with an alpha of 0.05 for pair-wise comparisons, and ordinary one-way ANOVA for comparisons of more than two samples, followed by Tukey's multiple comparisons tests. ANOVA, calculated using 'Imer()' in R (version 3.6.3), was used to analyze any differences in the number of viable progeny 
among strains. Hypothesis testing was performed with the 'car' package (47) using Type II sums of squares. Residual data points were normally distributed.

\section{Pseudomonas aeruginosa survival assay}

PA14 was streaked on standard Luria-Bertani (LB) plates supplemented with $100 \mu \mathrm{g} / \mathrm{ml}$ carbenicillin and grown overnight at $37^{\circ} \mathrm{C}$. Single colonies were picked and grown in $3 \mathrm{ml}$ of LB overnight. $20 \mu$ l of PA14 was seeded on $3.5 \mathrm{~cm}$ slow killing (SK) NGM plates as previously described (49). These SK plates were subsequently incubated overnight at $37^{\circ} \mathrm{C}$ and then equilibrated for 2 days at $25^{\circ} \mathrm{C}$. All strains used for the PA14 survival assay were grown to the gravid adult stage on $3.5 \mathrm{~cm}$ NGM plates at $20^{\circ} \mathrm{C}$ and bleached. Synchronized progeny were then grown to the L4-stage on NGM plates at $20^{\circ} \mathrm{C}$. Approximately $50 \mathrm{~L} 4 \mathrm{~s}$ were picked to SK plates and moved to $25^{\circ} \mathrm{C}$. Worms were transferred to new SK plates every $24 \mathrm{hr}$. Dead worm carcasses were counted at $48 \mathrm{hrs}$, and both carcasses and live worms were counted at $72 \mathrm{hr}$.

\section{Male mating rescue assay}

$\mathrm{N} 2$ and mutant hermaphrodites were reared at $20^{\circ} \mathrm{C}$. Ten $\mathrm{L} 4$ hermaphrodites of each genotype were transferred to plates with either three or zero N2 males, and maintained at $25^{\circ} \mathrm{C}$. After 24 hours, and at every 24 hours thereafter, the adult hermaphrodite was transferred to a fresh plate (males were removed), and the number of larvae was scored the following day until no more progeny were produced. Embryos/oocytes were imaged on an Olympus SZX16 Fluorescent Stereomicroscope with a Nikon DS-Fi1 camera. At 60 hours post temperature shift plates were assessed for the presence of male progeny to indicate successful mating.

\section{Antibodies used in this study:}

\begin{tabular}{|l|l|l|}
\hline Antibody & Reference & Name \\
\hline Rabbit anti-CSR-1 (total) & Claycomb et al., 2009 & $49-$ C1 \\
\hline
\end{tabular}




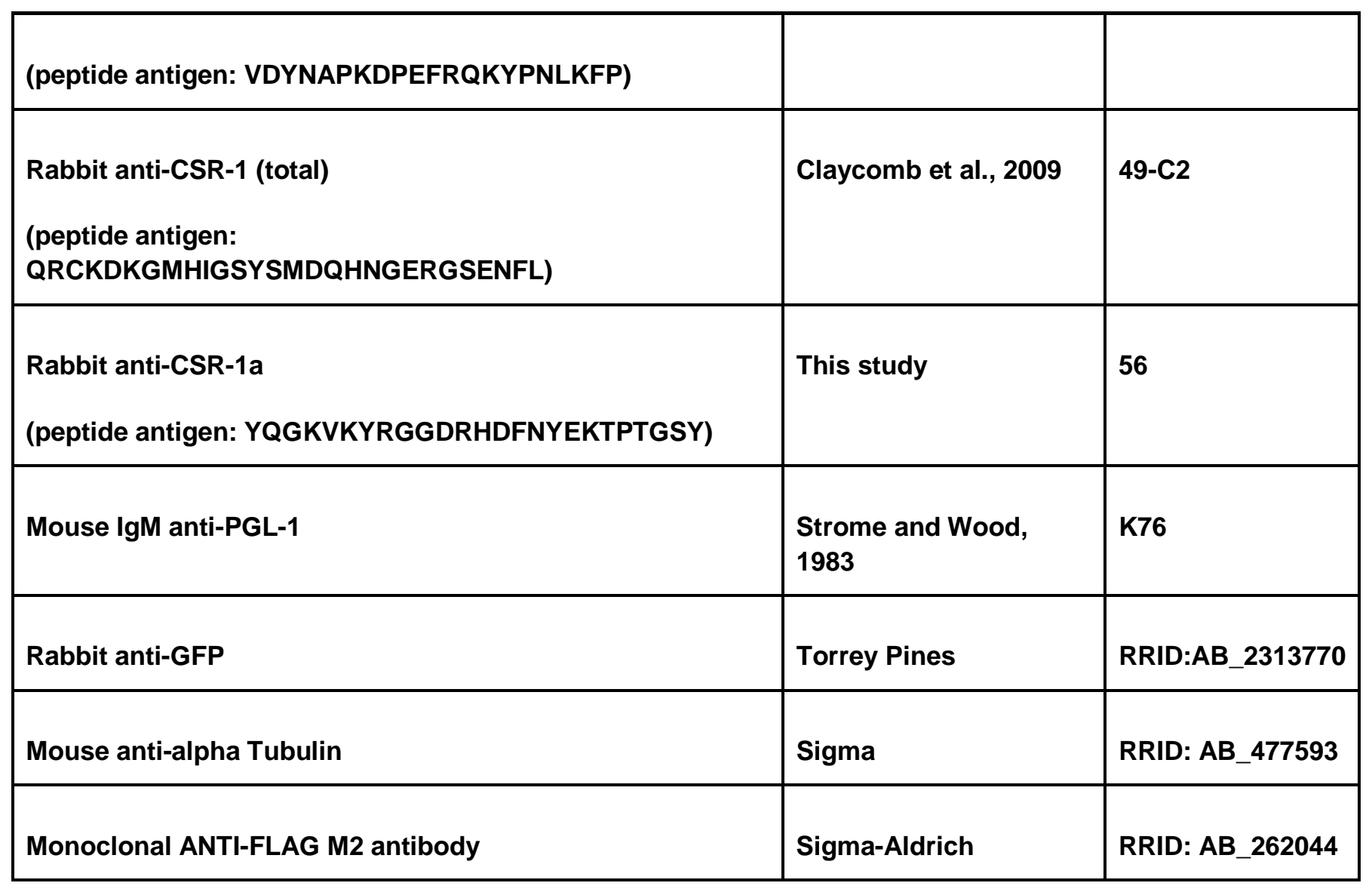

\section{Protein lysate preparation}

Synchronous populations of animals were grown at a density of approximately 100,000 animals per $15 \mathrm{~cm}$ plate on NGM with 5x concentrated OP50 E.coli, harvested at staged time points in 1x M9 buffer, and washed with $\mathrm{dd}_{2} \mathrm{O}$. The worm pellets were flash frozen in a dry ice/ethanol bath and stored at $-80^{\circ} \mathrm{C}$. The frozen pellets were resuspended $1: 1(\mathrm{v} / \mathrm{v})$ in ice-cold DROSO complete buffer $(30 \mathrm{mM}$ Hepes, 100mM Potassium Acetate, 2mM Magnesium Acetate, 0.1\% Igepal CA 630 (Sigma-Aldrich), 2mM DTT, 4x concentration Complete Protease Inhibitor (Roche) and 1\% (v/v) Phosphatase Inhibitors 2 and 3 (Sigma-Aldrich)) or EDTA Complete buffer (30mM Hepes, 100mM Potassium Acetate, 10mM EDTA, $10 \%$ Glycerol, $0.1 \%$ NP-40 or Igepal CA630 (Sigma-Aldrich), 2mM DTT, 1 tablet/5ml Protease inhibitor (Roche), 1:100 Phosphatase inhibitor 2 (Sigma-Aldrich), 1:100 Phosphatase inhibitor 3 (Sigma-Aldrich) and homogenized either with a stainless steel dounce (Wheaton Incorporated) until worms and embryos were no longer visible under the microscope or by sonication (Bioruptor Pico) for 10 cycles of $30 \mathrm{sec}$ ON/30sec OFF. In cases where RNA was to be isolated from immunoprecipitations 
using the protein lysates, $1 \%(\mathrm{v} / \mathrm{v})$ SUPERaseln RNase Inhibitor (ThermoFisher) was added to the buffer prior to homogenization. Lysates were centrifuged at $13,000 x g$ for $10 \mathrm{~min}$ at $4^{\circ} \mathrm{C}$, and the supernatant transferred to a fresh tube. A Lowry Assay (Bio-Rad) was used to determine the total protein concentration using a Nanodrop 1800C spectrophotometer.

\section{Western blotting}

Samples (typically 10-40 $\mathrm{gg}$ of protein lysate) were resolved by SDS-PAGE on precast gradient gels (4-12\% Bis-Tris Bolt gels, ThermoFisher) and transferred to Hybond-C membrane (Amersham Biosciences) using a Bio-Rad semi-dry transfer apparatus set at $25 \mathrm{~V}$ for $45 \mathrm{~min}$. Membranes were washed $3 \times 5 \mathrm{~min}$ with PBST $(0.1 \%$ Tween-20 in PBS) and blocked for 1 hour at room temperature with $5 \%$ milk-PBST (PBST with 5\% w/v dried nonfat milk). Membranes were incubated overnight at $4^{\circ} \mathrm{C}$ in primary antibodies (Antibodies Table). Membranes were washed $3 \times 10 \mathrm{~min}$ in PBST, blocked with $5 \%$ milk-PBST for 1 hour at room temperature, then incubated for 1 hour with secondary antibodies conjugated to HRP (Cell Signaling Technology). Membranes were washed 3 x5min in PBST and visualized using Luminata Forte Western HRP substrate (Sigma-Aldrich). Primary antibodies for FLAG [1mg/ml], CSR-1 (antibody 49C1) [0.25 mg/ml] were used at 1:3000 and CSR-1a (antibody 56) [0.25$0.57 \mathrm{mg} / \mathrm{ml}$ ] was used at 1:1000. Secondary antibodies listed in the Antibodies Table [1 mg/ml] were used at a concentration of 1:1000.

\section{Immunoprecipitation}

For each IP and co-IP experiment, 5mg total protein lysate was used per reaction. For small RNA cloning 3 to $5 \times 5 \mathrm{mg}$ IPs were performed and beads were pooled at the final wash step. GFP-Trap Magnetic Agarose beads (ChromoTek) equilibrated in EDTA Complete buffer were used for anti-GFP IPs and RFP-Trap beads or unconjugated beads (ChromoTek) were used for the Mock IP control. Dynabead Protein G beads (Invitrogen) were used for all non GFP/RFP IPs. CSR-1 rabbit peptide antibodies used for each IP were 49C2 [1.25 mg/ml] for total CSR-1 and 56 [0.25-0.57 mg/ml] for CSR1a (4). Dynabeads were resuspended in PBS with $0.1 \%$ Tween and incubated while rotating with $5 \mu \mathrm{g}$ 
of CSR-1 antibody (or rabbit lgG for the matched Mock IP control) for 10min at RT, then washed 3 x5min with EDTA complete buffer. Protein lysates were incubated with $25 \mu$ l of bead slurry for 1 hour while rotating at $4^{\circ} \mathrm{C}$. Beads were then washed with EDTA Complete buffer at least $5 \times 10 \mathrm{~min}$ each. Protein was eluted from the beads and denatured by incubation with sample buffer (4X Bolt Buffer with reducing agent - ThermoFisher) for $10 \mathrm{~min}$ at $70^{\circ} \mathrm{C}$. Input samples were prepared from the same lysate and diluted to a concentration of $2 \mathrm{mg} / \mathrm{ml}$ in sample buffer. For small RNA library samples, $10 \%$ of the total IP was taken for western blot to determine efficacy. The remaining sample was mixed with $4 \mathrm{x}$ TriReagent (Molecular Research Center, Inc.) and RNA was extracted as described (4).

\section{Small RNA library construction}

RNA for sRNA libraries, was isolated from either total worm lysate (input) or IP samples. Up to $4 \mu \mathrm{g}$ of RNA from the IP samples were treated with 5' polyphosphatase (Lucigen) for $30 \mathrm{~min}$ at $30^{\circ} \mathrm{C}$ followed by a standard phenol:chloroform:isoamyl alcohol extraction and ethanol precipitation. $1 \mu \mathrm{g}$ of total RNA from each sample was used for sRNA library preparation using the NEBNext Small RNA Library Kit for Illumina (New England Biolabs). The resulting PCR product was visualized using 10\% PAGE stained with SYBRGold (Life Technologies) and a region of 140-160bp was excised. DNA was eluted overnight at room temperature elution buffer (3.3M NaCl, 100mM Tris pH 7.5, 1mM EDTA), and precipitated with $20 \mu \mathrm{g}$ glycogen and $1 \mathrm{X}$ volume isopropanol overnight at $-20^{\circ} \mathrm{C}$. The resulting DNA was resuspended in $20 \mu \mathrm{l}$ Ultrapure ${ }^{\mathrm{TM}}$ water, and quantified using a Qubit HS DNA kit (ThermoFisher). All samples were pooled in equal amounts into a $20 \mathrm{nM}$ solution and sequenced as $50 \mathrm{bp}$ single end reads on an Illumina HiSeq 2500 or Miniseq At least two biological replicates were prepared for each sample.

\section{mRNA library construction}

Synchronized staged animals were collected in $1 \mathrm{X} M 9$ and washed in 3x in M9 and 1x in $\mathrm{ddH}_{2} \mathrm{O} .4 x$ pellet volumes of Tri-Reagent (Molecular Research Center, Inc.) were added to samples, and samples were flash frozen in a dry ice/ethanol bath, then stored at $-80^{\circ} \mathrm{C}$ for later RNA extraction. Total RNA extraction and ethanol precipitation were performed as described (4), followed by 
quantification using a Nanodrop $1800 \mathrm{C}$ spectrophotometer. $2 \mu \mathrm{g}$ of each sample was treated with

DNase I (Ambion), phenol:chloroform extracted, ethanol precipitated, resuspended in Ultrapure ${ }^{\mathrm{TM}}$

Distilled Water (ThermoFisher) and quantified using a Qubit HS RNA kit (ThermoFisher). $1 \mu \mathrm{g}$ of total

DNase treated RNA was used for library preparation using the NEBNext Ultra II Directional RNA Kit for Illumina with Poly-A selection, according to the manufacturer's manual. Samples were quantified with the Qubit HSDNA kit (ThermoFisher). Libraries were pooled in equal amounts to 20nM final concentration and sequenced 50bp single end reads on an Illumina HiSeq 2500 Sequencing System. For each genotype and temperature, three biological replicates were prepared.

\section{Data analysis}

\section{Small RNA Enrichment Analysis}

Raw read quality was assessed via FASTQC (version 0.11.5;

http://www.bioinformatics.babraham.ac.uk/projects/fastqc/) and adapters were trimmed via Cutadapt (version 1.15) (50) using the adapter sequence

"AGATCGGAAGAGCACACGTCTGAACTCCAGTCAC". Any reads that were not trimmed and were outside of the 16-30 nucleotide range were discarded (\$ cutadapt -a adapter sequence -f fastq -m 16 M 30 --discard-untrimmed). Reads were then mapped to the $C$. elegans genome (WormBase release WS262) using STAR-aligner (version 2.6.0c) (51) with the following command:

\$ --runThreadN 12 --outSAMtype BAM SortedByCoordinate --outFilterMultimapNmax 50 -outFilterMultimapScoreRange 0 --outFilterMismatchNoverLmax 0.05 --outFilterMatchNmin 16 -outFilterScoreMinOverLread 0 --outFilterMatchNminOverLread 0 --alignIntronMax 1.

A custom R script (version 3.6.3) was used to count the number of reads for each feature (e.g. protein coding genes, pseudogenes, etc.). WormBase version WS262 PRJNA13758 ce11 canonical geneset annotations were used (excluding miRNAs). C. elegans miRNA annotations were obtained from miRBase (release 22.1). To determine targets enriched for sRNAs in Argonaute IPs, reads were first normalized to library size (reads per million - RPM). Enrichment was defined as genes having a minimum of 5 RPM in both IP samples, and greater than or equal to 2 fold enrichment in both IPs 
relative to Input samples. Similarly, sRNA-seq data were determined to be depleted (15) by the presence of $>5$ RPM in Input, and greater than or equal to 2 fold decrease in the number of reads in the mutant versus Input. All sRNA samples, including those published by other groups were analyzed using our own computational pipeline for consistency.

The size and first nucleotide distribution plots and enrichment scatter plots were made using the ggplot2 package (48). Heatmaps were generated using the pheatmap package (https://CRAN.Rproject.org/package=pheatmap). Venn plots were made using VennDiagram package (https://CRAN.Rproject.org/package=VennDiagram) and VennDetail Package 1.2.0

(https://github.com/guokai8/VennDetail). Statistical analysis for all Venn-pie Gene set enrichment comparisons was conducted using Fisher's Exact Test. The overlap between enriched sRNAs and various published gene sets was determined and plotted using the VennDetail Package (https://github.com/guokai8/VennDetail) using percentage parameters. Additional genes and gene groups of interest were examined using WormMine $(52,53)$, WormExp $(54)$ and WormBase Enrichment Suite (55). The significance in overlap between gene sets was calculated using Fisher's Exact Test. Non-templated nucleotide additions to sRNA 3' ends were identified and quantified using a custom script. Metagene plots of sRNA distributions along target genes were made using a custom script. Replicates were aggregated and normalized by RPM. For clustering diagrams, sRNA targets were grouped via hierarchical clustering by binary distance using the pheatmap package (https://CRAN.Rproject.org/package=pheatmap).

\section{mRNA Differential Gene Expression Analysis}

mRNA analysis was conducted in the same way as the small RNA analysis described above, except that reads were trimmed with the following command (retaining only reads without adapters): \$ cutadapt -a adapter sequence -f fastq -m 51 -M 51. Differential expression and statistical analysis were performed using DESeq2 (56) and significantly-changed genes were designated by the $p$-adjusted value of $<0.1$. Gene overlaps and enrichment set analyses were conducted as described above. 


\section{Gene Ontology Analysis}

Gene lists generated via our custom pipelines were subjected to Gene Ontology Analysis using the WormBase Enrichment Suite (55).

\section{Sequence Alignment and Cladogram Construction}

csr-1 sequences for other Caenorhabditis species were obtained from the NCBI database (57), then aligned with MUSCLE (58) and custom coloured to highlight particular amino acids relevant to this study in SeaView (59). The cladogram was constructed based on the alignment.

\section{RESULTS}

\section{A screen for proteasomal regulators uncovers csr-1a alleles}

The csr- 1 locus encodes two isoforms that vary by the addition of 163 amino acids to the Nterminal region of CSR-1a. In contrast to the rest of the CSR-1 protein, the N-terminal region is poorly conserved among related nematode species, with the notable exception of the RG motifs (Fig. 1A-B). We became interested in the two isoforms of CSR-1 for two major reasons. First, several reports demonstrated that the two isoforms of CSR-1 were differentially expressed throughout development, suggesting they could play different roles in gene regulation in different tissues $(4,29)$. Second, a forward genetic screen for factors that regulate the proteasome pathway serendipitously uncovered several alleles that only affect csr-1a, suggesting a somatic function specifically for this isoform (Fig. S1). In this screen, we generated a genome-integrated repetitive transgene array that drives GFP expression under control of the promoter of the rpn-2 gene (rpn-2::gfp). rpn-2 encodes the C. elegans orthologue of Rpn2/PSMD1, a non-ATPase subunit of the 19S proteasome that is essential for viability of eukaryotic cells (Fig. S1A) (60). For reasons that are not entirely clear, the rpn-2::gfp fusion gene was generally silenced throughout the worm, but could be activated in the intestine by proteasome inhibitors or mutations in proteasome subunits, like another proteasomal fusion gene rpt-3::gfp (data not shown and (40)). EMS mutagenesis of this strain yielded eleven mutants that showed elevated expression of GFP in intestinal cells, including mutations in: rrf-1, rsd-2 and rde-1, which encode RNAi 
pathway factors (61-63); four independent alleles of csr-1a that lie within the first exon; and four alleles which remain uncharacterized (Fig. S1B-E). We verified the effects of these mutations with independent alleles (data not shown).

All of the csr-1a alleles recovered cause amino acid substitutions at conserved glycine residues (G91R, two alleles of G120E, and G124E) within or nearby conserved RGG/RG repeats of the Nterminus (Fig. 1B, S1C). We hypothesized that CSR-1a and the other RNAi factors recovered may play a role in sRNA-mediated silencing of the rpn-2::gfp transgene, therefore we asked whether CSR-1a associates with transgene-derived sRNAs via CSR-1a Immunoprecipitation (IP) with a CSR-1-specific antibody, followed by sRNA sequencing. In parallel, we assessed whether transgene-derived sRNAs were depleted in csr-1a mutants, as loss of AGOs generally destabilizes sRNA pools. We observed no enrichment of transgene sRNAs in CSR-1a complexes, nor did we detect a depletion of transgene sRNAs in the csr-1a mutant strain, suggesting that the role for CSR-1a in regulating rpn-2::gfp expression was not sRNA-mediated, and could be indirect (Fig. S1F-G). Despite these results, this screen left us with a rich set of alleles to assess the isoform-specific functions of CSR-1a throughout development.

\section{CSR-1 isoforms have distinct expression patterns throughout development}

In addition to the csr-1a alleles recovered in our screen, we generated an extensive set of csr-1 mutants and epitope-tagged strains using CRISPR-Cas9 genome editing $(36,64)$ and MiniMos single copy transgene insertions (41) (Fig. 1C). We introduced GFP::3xFLAG into the N-terminus of the long isoform, CSR-1a, via CRISPR-Cas9 genome editing at the endogenous csr-1 locus (36). Because the $c s r-1 b$ locus is entirely shared with the csr-1a locus, epitope tagging CSR-1b invariably results in the internal epitope tagging of CSR-1a, thus we used this to our advantage to epitope tag both isoforms in a single strain (37). However, having both isoforms tagged precludes our ability to study CSR-1b separately. Therefore, we also introduced a stop codon into the first exon of csr-1a, to generate a third strain that is a null mutant for csr-1a, and expresses only GFP::3xFLAG::CSR-1b (referred to as csr1a(tor159) in figures where csr-1a loss of function is assessed). 
We evaluated the tissues and developmental time points in which each isoform is expressed using western blotting (Fig. 1D) and confocal microscopy (Fig. 1E, 2, 3A, S2-S3). In these experiments, CSR-1a and CSR-1b displayed clear differences in the timing and tissue-specificity of expression. Western blotting on the GFP::3xFLAG::CSR-1b strain demonstrated that, consistent with published data, CSR-1b is expressed during all developmental stages in hermaphrodites, with the highest levels of protein present in adults and in embryos (Fig. 1D) (4). In contrast, CSR-1a displays a more restricted expression profile, and is detected only in L4, young adult, and gravid adult developmental stages (Fig. 1D).

In examining each strain by confocal microscopy throughout development, we observed that CSR-1b is constitutively present in the germline at all developmental stages in hermaphrodites, including the primordial germ cells in embryos, the spermatogenic gonad, and the oogenic gonad (Fig. 1E, 2A, S2). Consistent with expression during spermatogenesis in hermaphrodites (Fig. 2A), CSR-1b is also expressed in the male gonad (Fig. S2). In contrast, CSR-1a is only detected in the proximal germline at the L4 larval stage in hermaphrodites, coincident with sperm production (Fig. 1E, 2A, S3). CSR-1a is also expressed in the male gonad, similar to its expression pattern in L4 hermaphrodites (Fig. S3).

Within all germline tissues, CSR-1b is present primarily in the cytoplasm and robustly localizes to germ granules (Fig. 1E, 2A-B, S2). Similarly, in the L4 germline, CSR-1a is also present in the cytoplasm and enriched in germ granules (Fig. 2A-B, S3, 9A). CSR-1 had previously been shown to localize to P granules (4), but in the meanwhile, several other types of germ granules, including Z granules have been identified (65). To determine which type of germ granules each CSR-1 isoform associates with, we crossed RFP-tagged versions of PGL-1 (a P granule marker) and ZNFX-1 (a Z granule marker) into the GFP::3xFLAG::CSR-1a/b strains and determined the extent of pixel overlap in L4 and young adult hermaphrodite germlines. We found that both isoforms of CSR-1 overlap with PGL1 and ZNFX-1 to a similar extent in the L4 germline and that CSR-1b overlaps with both PGL-1 and ZNFX-1 in the oogenic germline (Fig. 2B, see Fig. 8E for quantification). 
Because of their germline expression patterns, we next explored whether either CSR-1 isoform could be transmitted to progeny via sperm (CSR-1a, b) or oocytes (CSR-1b only). First, we performed confocal microscopy on sperm released from males. We observed that much of the CSR-1b pool was present in the residual body, a structure where sperm cytoplasmic contents are discarded during spermatogenesis (Fig. 2C) (22). We also detected CSR-1b in mature spermatids, where it was present both in the nucleus and cytoplasmic foci (Fig. 2C) (14). In contrast, CSR-1a is not segregated into the residual body, nor is it packaged into mature spermatids. These observations indicate that CSR-1b alone has the potential to be passed from males to progeny via sperm.

In oocytes, CSR-1b localizes to the cytoplasm, germ granules, and oocyte chromatin (Fig. 2A, 2B, 2D), and therefore could be maternally provisioned $(4,66)$. In self-fertilizing GFP::3xFLAG::CSR-1b hermaphrodites, we observed GFP expression in early embryos, prior to the onset of zygotic transcription (Fig. 2E). In contrast, we did not observe GFP expression in early embryos produced from crosses between GFP::3xFLAG::CSR-1 males to wild-type hermaphrodites (Fig. 2F). Instead, we first detected zygotic GFP::3xFLAG::CSR-1 in the primordial germ cells, Z2 and Z3. Finally, during early embryogenesis until approximately the 50 cell stage, maternally provisioned CSR-1b is detected in both somatic and germ cells (Fig. 2E, 2G). In both cell types, CSR-1b is present in the cytoplasm and localizes to the nucleus in a cell-cycle dependent manner, coincident with mitosis (Fig. 2G). Collectively, these data indicate that CSR-1b is maternally, and potentially paternally inherited, and that zygotic expression of CSR-1b initiates when the primordial germ cells become transcriptionally active during late embryonic development. In contrast, CSR-1a is not maternally or paternally inherited, and is not expressed in embryos.

In adult hermaphrodites, we were unable to detect CSR-1a in the oogenic germline (Fig. 2A-B 2D, S3), nevertheless, western blotting data demonstrated the presence of CSR-1a in adults (Fig. 1D). This suggested that CSR-1a may be expressed in the soma. Indeed, we observed CSR-1a in several adult somatic tissues of both hermaphrodites and males (Fig. S3), including the intestine (Fig. 3A), spermatheca (Fig. S3), and several cells within the tail (Fig. S3). In adult males, CSR-1a is also present in the vas deferens and seminal vesicle (Fig. S3). In fact, the earliest expression of CSR-1a was in the 
intestine from the L3 stage onward (Fig. 1C). In contrast, CSR-1b was not observed in any postembryonic somatic tissues.

Consistent with this intestinal expression pattern, we observed enrichment of the intestinal master transcription factor ELT-2 (67) upstream of the csr-1a transcription start site at the L3 stage of development by examining existing transcription factor binding data (Fig. 3B) (68). The first intron of the csr-1 locus possesses potential regulatory elements for csr-1b. Within this intron, we did not observe ELT-2 binding, but instead observed enrichment of several germline enriched transcription factors, including members of the SNAP-C (69) and DRM complexes (70), which we predict regulate the expression of $c s r-1 b$ in the germline. Taken together, these results highlight the potential for CSR-1a to play novel roles during spermatogenesis and within specific somatic tissues, including the intestine.

\section{CSR-1b is the essential CSR-1 isoform but CSR-1a can compensate}

csr- 1 is the only singly essential ago in C. elegans. Homozygous zygotic null mutants of csr-1 (which affect both isoforms) that are generated from heterozygous hermaphrodites develop to adulthood, but display defects in oogenesis $(3,4)$. Consequently, they produce few embryos, and those few embryos that are generated display chromosome segregation defects and arrest early in embryogenesis, prior to the 50 cell stage $(4,11)$. In contrast, csr- 1 null mutant males are fertile under normal lab culture conditions, but display a progressive loss of fertility over several generations at a stressful temperature $\left(25^{\circ} \mathrm{C}\right)(14)$. The different expression profiles of CSR-1 isoforms in the germline suggest that each isoform could be responsible for different facets of fertility. For instance, because CSR-1a is not expressed in the hermaphrodite germline or in early embryos, we expected that loss of csr-1a would likely have little impact on oocyte development or embryo viability. Therefore, we asked whether either isoform was sufficient for fertility, or if both were redundantly required for proper germline development and gamete function.

We assessed the fertility of our set of csr-1a mutants and epitope tagged strains, along with two strains in which csr-1 null mutants were rescued with ubiquitous expression of either CSR-1a or CSR1b ( Fig. 4). First, we verified that each of our GFP::CSR-1a, GFP::CSR-1b, and GFP::CSR-1 strains 
displayed wild type fertility under the normal lab culture temperature of $20^{\circ} \mathrm{C}$ (Fig. $\left.4 \mathrm{~A}-\mathrm{B}\right)$. Next, we tested whether these strains displayed any fertility defects under the stressful temperature of $25^{\circ} \mathrm{C}$. At $25^{\circ} \mathrm{C}$, GFP::CSR-1b, which also possesses a null mutation in csr-1a (csr-1a(tor159)), displayed a slight but reproducible reduction in viable brood size (Fig. 4A-B). We also tested a strain possessing only the $c s r-1$ a premature stop allele, but no gfp insertion in $\operatorname{csr}-1 b\left(\operatorname{csr}-1 a^{\left[G 120^{*}\right.}\right)$, as well as a missense allele affecting a conserved RG repeat of the CSR-1a recovered from the proteasomal regulators screen (csr$1 a^{[G 91 R]}$ (Fig. 1B). While csr-1a ${ }^{\left[G 120^{*}\right]}$ was a null allele, CSR-1a[G91R] was expressed at slightly lower levels than wild-type CSR-1a, but localized to the intestine and P granules normally (Fig. S4A-D). Consistent with our results using csr-1a(tor159), both of these alleles displayed a normal brood size at $20^{\circ} \mathrm{C}$, and reduced brood size at $25^{\circ} \mathrm{C}($ Fig $4 \mathrm{C})$.

The subtle and temperature-dependent fertility defect led us to pursue the role of $c s r-1 a$ at $25^{\circ} \mathrm{C}$ further. Because CSR-1a germline expression occurs only during spermatogenesis, and loss of both CSR-1 isoforms in males causes a progressive loss of fertility over many generations (14), we tested whether loss of CSR-1a causes a progressive decline in fertility. We cultured the csr-1a(tor159) null mutant at $20^{\circ} \mathrm{C}$ for $>25$ generations, then shifted it to $25^{\circ} \mathrm{C}$ to perform brood analysis. In parallel, we freshly outcrossed these "late generation" csr-1a(tor159) null mutants, shifted them to $25^{\circ} \mathrm{C}$ and assayed their brood size in parallel. We found that csr-1a(tor159) null mutants in culture for many generations displayed a smaller brood size than wild-type worms at $25^{\circ} \mathrm{C}$, but that this phenotype was reversed by outcrossing to wild-type (N2) before selfing (Fig. 4D). These data suggest that loss of CSR1a from the spermatogenic germline results in progressive loss of fertility through a transgenerational epigenetic mechanism. This is reminiscent of transgenerational epigenetic inheritance defects displayed by other germline ago mutants, including csr-1(tm892) null mutant males (14).

Next, we asked if ubiquitous expression of either CSR-1 isoform is sufficient to rescue the csr1(tm892) null mutant. To do this, we constructed single copy genomically-integrated transgenes, in which the coding sequence of each csr-1 isoform was tagged with gfp and was expressed using a constitutive rpl-28 promoter and csr-1 3'UTR in a csr-1(tm892) null mutant background (Fig. 1B). Remarkably, both strains were viable, indicating complementation (Fig. 4E). We measured the extent to 
which expression of each isoform rescued fertility by performing total brood counts versus viable brood counts. In contrast to csr-1(tm892), which produces very few embryos, the csr-1(tm892) strain possessing the $r p l-28:: g f p:: c s r-1 b$ transgene produced $73.1 \%$ the total brood of wild-type worms (data not shown). However, despite this robust brood size, we found that many of the embryos were not viable (Fig. 4E; csr-1b rescue viability was $47.4 \%$ of wild-type worms), and died late in embryogenesis as malformed larvae (Fig. S4F). This embryonic arrest occurs later in development than embryos produced by csr-1(tm892) homozygous hermaphrodites, suggesting that the phenotype could be due to different causes, including the developmental timing and cell types in which CSR-1b was expressed via this ubiquitous promoter, or the level to which it was expressed. The brood size may also be reduced due to a lack of CSR-1a in this strain.

To ensure that only csr-1a was expressed in the second transgenic rescue strain, we eliminated the first intron. Western blotting on protein lysates from this strain showed that only GFP::CSR-1a was expressed from this transgene and there was no CSR-1b protein present (Fig. S4E). By using this constitutive rpl-28 promoter, CSR-1a is expressed in many tissues and at developmental time points where and when it would not normally be expressed, including in the oogenic germline and in early embryos (Fig. 4F). Remarkably, this strain displayed a viable brood size comparable to wild type (Fig. 4E), indicating that GFP::CSR-1a can compensate for loss of csr-1b when ectopically expressed in the oogenic germline and in embryos. Overall, this result was unexpected, and suggests that CSR-1a can execute the molecular functions of CSR-1b, but has differentiated itself from CSR-1b by its expression profile, and potentially other features of the protein. Conversely, the reduced brood size in the CSR-1bexpressing rescue strain may indicate that CSR-1b cannot perform the functions of CSR-1a, or that the timing, cell types, and levels of CSR-1b are not optimized in this strain.

By integrating these observations with our knowledge of CSR-1 expression profiles, we conclude that under normal culture conditions, CSR-1a is not required for embryonic viability. Instead $c s r-1 b$ plays an essential role during oocyte development and in the early embryo. However, under stressful conditions, such as elevated temperature, csr-1a may play a role in fertility that is likely linked to a role in sperm development and differentiation (see below). 


\section{CSR-1a and CSR-1b associate with 22G-RNAs to target partly overlapping sets of protein coding}

\section{genes}

To understand whether each CSR-1 isoform has distinct or overlapping target transcripts and roles in gene regulation, we set out to determine the pools of sRNAs bound by each isoform. Past studies have shown that CSR-1 associates with 22G-RNAs, and 22G-RNAs display perfect complementarity to target transcripts $(4,14,15)$. Therefore, by determining the sRNA complements associated with each CSR-1 isoform, we can computationally identify gene targets. In these experiments, we used GFP antibodies to perform immunoprecipitation (IP) of GFP::3xFLAG::CSR-1 (for CSR-1a, CSR-1b and CSR-1) in synchronized L4 worm populations maintained at $20^{\circ} \mathrm{C}$ (Fig. 5 , S5). We chose L4 worms because both isoforms are robustly expressed at this stage. For CSR-1b we expected that SRNA populations would reflect germline activities during spermatogenesis. For CSR-1a, we anticipated that we would recover both somatic and germline (spermatogenesis) sRNA populations, due to its expression profile. In parallel, we cloned and sequenced total sRNA samples from the same samples (Input). Two replicates were performed for each condition (Supp. Table S1-2).

We focused our analysis on the CSR-1a and CSR-1b IPs (Fig. 5A). (Note that total CSR-1 results are presented in Fig. S5, but not discussed further.) We first examined the size and first nucleotide distribution of sRNAs from the Input and IP samples, and observed a large proportion of 22G-RNA reads present in each CSR-1 IP (Fig. 5B, Supp. Table S1-2). Next, we determined which reads were enriched in each GFP::3xFLAG::CSR-1 IP relative to Input (with a minimum of 5 reads per million (RPM) and a 2-fold or greater increase in IP versus Input) to define a set of target transcripts (genes for which antisense sRNAs are enriched in the IP) for each isoform (Fig. 5C, Supp. Table S2). Protein coding genes are the major targets of both isoforms (CSR-1a: 2572 genes; CSR-b: 5326 genes out of 20116 total genes in the genome) (Fig. 5C). Both isoforms also target a small number of transposable elements (CSR-1a: 6 TE; CSR-b: 7 TE out of 285 total TE) and pseudogenes (CSR-1a: 59 pseudogenes; CSR1-b: 32 pseudogenes out of 1885 total pseudogenes) (Fig. 5C). While there is extensive overlap between the CSR-1a and CSR-1b protein coding gene targets (1757 genes), there 
are also distinct sets of genes that are specifically targeted by one isoform (815 for CSR-1a, 3569 for CSR-1b) (Fig. 5D).

We examined how the CSR-1 shared and isoform-specific 22G-RNA targets overlap with those of other sRNA pathways by comparing them to previously published $R d R P$ mutant, ago mutant, and AGO IP/sRNA-seq datasets (Fig. 5E) $(4,14,15,71)$. Such comparisons could provide insights into any differences in 22G-RNA biogenesis pathways between the CSR-1 isoforms and their distinct and overlapping target gene sets. Striking patterns emerged from these comparisons. First, we found that CSR-1b-specific targets and CSR-1a/b shared targets are over-represented in genes that are depleted of 22G-RNAs in ego-1 mutants. In contrast, CSR-1a-specific targets are over-represented in genes that are depleted of sRNAs in rrf-1 and rrf-3 mutants, while CSR-1b-specific targets are not. CSR-1a/b shared targets are also over-represented in genes depleted of sRNAs in rrf-3 mutants (Fig. 5E). The overlap with different RdRPs reflects expression profiles: RRF-1 is mostly expressed in the soma (like CSR-1a), EGO-1 is expressed constitutively in the germline (like CSR-1b), and RRF-3 is important during spermatogenesis (like CSR-1a and CSR-1b) $(4,14,15,23,27,71)$. Consistent with a link to spermatogenic sRNA pathways (below), CSR-1a-specific and CSR-1a/b shared targets are overrepresented in both the group of genes depleted of sRNAs in alg-3; alg-4 spermatogenesis-specific ago mutants (Fig. 5E). Interestingly, CSR-1b-specific and CSR-1a/b shared targets are over-represented in existing CSR-1(total) targets from males and hermaphrodites, while CSR-1a-specific targets are underrepresented in these sets. This likely reflects the relative abundance of each isoform, as CSR-1b is expressed at higher levels than CSR-1a and can be more easily immunoprecipitated by CSR-1 antibodies. Of note, we observed a similar phenomenon with our GFP::3xFLAG::CSR-1 (both isoform) IPs (Fig. S5). All sets of CSR-1 target genes are under-represented in WAGO-1 targets, consistent with a functional distinction between the CSR-1 and WAGO-1 pathways (Fig. 5E) $(4,15)$. Collectively, these data suggest that different biogenesis mechanisms may contribute to the production of each subset of CSR-1 associated 22G-RNAs.

We asked if there are any differences in the distribution of sRNAs along the groups of target genes for each CSR-1 isoform. To assess this, we performed metagene analysis, examining the 
density of sRNAs along the set of CSR-1a vs. CSR-1b protein coding gene targets, after normalizing for gene length (Fig. 5F). CSR-1b-associated sRNAs displayed a pattern consistent with previous reports, in which sRNAs were enriched at the $3^{\prime}$ end of the target transcripts (72). However, we observed a distinct pattern of sRNAs associated with CSR-1a, in which sRNAs were distributed along the entire length of the target transcripts. This result is reminiscent of the pattern of WAGO-1 sRNAs, which are generated by the RdRP RRF-1 (15). Collectively, these observations highlight similarities and differences in CSR-1a vs. CSR-1b 22G-RNAs, and hint that, at least in some tissues, there may be differences in the CSR-1 22G-RNA biogenesis pathways.

\section{CSR-1a and CSR-1b target different subsets of germline genes}

To further characterize the overlapping and distinct 22G-RNA gene targets of each CSR-1 isoform, we categorized targets based on existing data that designate mutually exclusive sets of sperm, germline constitutive, and oocyte enriched transcripts (29) (Fig. 6A, Supp. Table S1-2). Because CSR1a and CSR-1b only overlap in their expression in the spermatogenic gonad, we anticipated that the set of shared targets would be enriched in germline genes. Indeed, we observed that germline constitutive and spermatogenic gene sets were over-represented among the shared targets, and accounted for nearly all 1757 of these shared targets, while oogenic genes were under-represented (Fig 6A). The CSR-1b specific targets were over-represented in the germline constitutive and oogenic gene sets. This reflects the constitutive germline expression pattern of CSR-1b and highlights its broad role in germline gene regulation. It is important to note that the CSR-1b specific targets are under-represented in spermatogenesis genes, highlighting that any spermatogenesis targets of CSR-1b are generally shared with CSR-1a. Finally, CSR-1a specific targets were over-represented in germline constitutive and spermatogenic gene sets, and under-represented in oogenic genes, again, reflecting its expression during spermatogenesis. Notably, approximately one-third of the CSR-1a specific targets (268/815 genes) did not fall into any of these germline categories, and were enriched for targets expressed in somatic tissues. Of these 268 genes, 171 were enriched in the intestine (Fig. 6B). 
Based on a previously described role for CSR-1 in regulating histones, we examined histone targeting by the CSR-1 isoforms in more detail. Replication dependent histone mRNAs are generally not poly-adenylated, but possess a 3' stem loop that must be cleaved for mRNA maturation and translation (73). CSR-1 has been implicated in histone mRNA processing in worms. In this mechanism, sRNAs guide CSR-1 to the stem loop cleavage site, where CSR-1 is proposed to act as the endonuclease (10). There are approximately 80 histone genes detectable in the $C$. elegans genome and we found that 50 of the histone genes are targeted by CSR-1a, CSR-1b, or both isoforms (Fig. 6CD, Supp. Table S2). CSR-1a targets Histones H2A, H2B, and H3, while CSR-1b targets predominantly H3. Histone $\mathrm{H} 4$ is a prominent target of both isoforms. Because both CSR-1 isoforms possess the same catalytic tetrad within the PIWI domain, it is plausible that both isoforms could process the 3 ' stem loop of different classes of histone genes in different tissues during development.

\section{Gene expression is perturbed in csr-1a mutants}

Our data collectively attribute the previously described expression pattern and essential functions of CSR-1 in the literature to CSR-1b, whereas CSR-1a plays an uncharacterized role that promotes fertility. We therefore focused our efforts on understanding how loss of CSR-1a affects the expression of its sRNA target genes to maintain fertility. Because csr-1a mutants exhibit temperaturedependent fertility defects after long-term culture, we performed mRNA-seq on late generation L4 stage csr-1a(tor159) mutants vs. wild-type worms at $20^{\circ} \mathrm{C}$ and $25^{\circ} \mathrm{C}$.

We first compared gene expression changes in csr-1a(tor159) mutants to wild-type worms at $20^{\circ} \mathrm{C}$. We found that very few genes that changed at this temperature were the targets of CSR-1a or CSR-1b 22G-RNAs (Fig. 7A, Supp. Table S2). In contrast, when we compared gene expression changes of $\operatorname{csr}$-1a(tor159) mutants to wild-type worms at $25^{\circ} \mathrm{C}$, we found that a greater number of genes display altered expression (Fig. 7B, Supp. Table S2). A large fraction of these mis-expressed genes are CSR-1a and/or CSR-1b targets. Interestingly, the majority (61\%) of genes that display reduced expression are CSR-1b targets (including both shared CSR-1 targets or CSR-1b specific targets). $66 \%$ of genes with reduced expression are targeted by either CSR-1 isoform. A smaller subset 
of genes with increased expression are targeted by either CSR-1 isoform (21\%), but a greater absolute number of genes are the targets of CSR-1a (355 up-regulated genes vs. 187 down-regulated genes). Overall, $63 \%$ of CSR-1a 22G-RNA targets are mis-regulated.

Tissue Enrichment and Gene Ontology (GO) analysis indicated that the genes that display decreased expression in csr-1a(tor159) mutants are expressed in the germline, reproductive system, and in males. GO terms including meiotic cell cycle, reproduction, chromosome segregation, and nucleotide binding were also enriched in this set, suggesting that these genes are involved in spermatogenesis (Fig. 7C, Supp. Table S2). Indeed, when we assessed the relative expression of germline constitutive, oogenic, and spermatogenic transcripts in wild-type and csr-1a(tor159) mutant worms, we observed that only spermatogenic genes were significantly decreased in the mutant (Fig. 7D, Supp. Table S2) (29). These results point to a positive gene regulatory role for CSR-1a in the spermatogenic gonad, and are consistent with the previously described positive, or licensing role for CSR-1 overall in regulating spermatogenic transcripts (14).

In sharp contrast, the genes that displayed increased expression in csr-1a(tor159) mutants at $25^{\circ} \mathrm{C}$ are largely expressed in the soma, including the intestine, where CSR-1a is expressed (Fig. 7E, Supp. Table S2). GO terms including response to biotic stimulus, stress response, interspecies interaction between organisms, and immune system process were enriched in this set, suggesting a role in pathogen responses. The intestine serves as an important interface with the environment, including pathogens. This prompted us to ask whether csr-1a(tor159) mutants respond better or worse than wild-type animals when faced with a pathogen, such as the gram-negative bacterium Pseudomonas aeruginosa (strain: PA14) (49, 74). To our surprise, we observed that csr-1a(tor159) mutants were able to survive better on PA14 than wild-type animals (Fig. 7F). We reason that the upregulation of immune response genes in csr-1a(tor159) mutants "primes" worms to better defend against PA14, and possibly other pathogens. Collectively, our gene expression data indicate that CSR1a promotes the expression of spermatogenesis genes in the L4 spermatogenic gonad. In contrast, somatic CSR-1a, likely acting in the intestine (soma), is required to dampen expression of immune and pathogen response genes. These roles are likely mediated, at least in part, by sRNA directed 
mechanisms. However, many of the mis-expressed genes are not direct targets of CSR-1a or CSR-1b, so it is important to note that indirect effects on gene expression are also possible.

\section{CSR-1b and CSR-1a differentially intersect with the WAGO-4 22G-RNA pathway}

Having established a positive gene regulatory role for CSR-1a alongside CSR-1b during spermatogenesis, we aimed to determine how CSR-1-dependent gene regulation intersects with other sRNA pathways during spermatogenesis. First, we assessed the germline AGO, WAGO-4.

Recent studies have demonstrated that WAGO-4 binds to a pool of 22G-RNAs that target nearly all of the previously defined CSR-1 protein coding gene targets (75). WAGO-4 is expressed constitutively in the germline, where it localizes to germ granules, including $Z$ granules in adults and $P$ granules in early embryos(65). WAGO-4 has been implicated in transgenerational epigenetic inheritance of exogenous RNAi, and its loss leads to a transgenerational loss of fertility at the stressful temperature of $25^{\circ} \mathrm{C}$ (Mortal germline, Mrt phenotype) $(65,75,76)$. Because of this link between the CSR-1 and WAGO-4 targets, we aimed to delineate the relationship between the targets each CSR-1 isoform and WAGO-4.

We cloned and sequenced sRNAs associated with GFP::3xFLAG::WAGO-4 from L4 worms, and compared these data to the targets of CSR-1a and CSR-1b (37). Our WAGO-4 IPs identified 22GRNAs that target 4933 protein coding genes, which overlap extensively with existing data sets (data not shown) (75). We asked if the targets of WAGO-4 overlap with CSR-1a or CSR-1b targets, and found that the greatest overlap was between CSR-1b and WAGO-4 targets (4499/5326 CSR-1b genes; 84.5\%) (Fig. 8A, Supp. Table S1-2). Among these were 1065 CSR-1a/b shared targets, which encompassed almost all of the CSR-1a and WAGO-4 overlap in targets. In fact, only 19 CSR-1aspecific targets overlapped with WAGO-4 targets. These data demonstrate that CSR-1b and WAGO-4 pathways intersect, while any relationship between the CSR-1a and WAGO-4 pathways is centered around CSR-1b shared targets, rather than CSR-1a-specific targets.

We next asked what types of germline target genes fall into each AGO group (Fig. 8B, Supp. Table S2). Here, the distinction is clear. CSR-1a and CSR-1b specific targets are both enriched in 
spermatogenic and germline constitutive genes, while the CSR-1 shared targets are enriched in spermatogenic genes only. In contrast, CSR-1b/WAGO-4 shared targets are enriched in oogenic and germline constitutive genes. Genes targeted by all three AGOs were enriched in germline constitutive genes. These data distinguish the CSR-1a, CSR-1b and WAGO-4 into two major germline regulatory modules: CSR-1a and CSR-1b target a set of spermatogenesis genes, while WAGO-4 operates with CSR-1b to target germline constitutive and, to a lesser extent oogenic transcripts.

To better understand the relationship between the CSR-1 isoforms and WAGO-4, we examined several other features of WAGO-4 22G-RNAs. First, we analyzed the pattern of 22G-RNAs along WAGO-4 targets using a metagene plot (Fig. 8C). Here, we observed that the WAGO-4 22G-RNA pattern appears very similar to the CSR-1b 22G-RNA pattern and, as we had observed for CSR-1b, is distinct from the CSR-1a 22G-RNA pattern (compare Fig. 8C to Fig. 5F). This may reflect different 22GRNA biogenesis mechanisms.

Next, we examined the extent to which WAGO-4 22G-RNAs display non-templated addition of uridine (Fig. 8D). CSR-1 22G-RNAs are known to be uridylated at their 3' ends by terminal uridyltransferase, CDE-1, and this modification was originally thought to facilitate turnover of CSR-1associated 22G-RNAs $(4,77)$. Conversely, WAGO-4 is thought to favor an association with the uridylated 22G-RNAs. Hence, the uridylation state of the 22G-RNAs may control the balance in association between CSR-1 and WAGO-4 (75). First, we assessed to what extent CSR-1a and CSR-1b 22G-RNAs were uridylated. We found that CSR-1b 22G-RNAs were consistently U-tailed $(24.14 \%$ of 22G-RNA reads in IP samples had one or more U's added vs. 7.01\% in Input samples). In contrast, CSR-1a 22G-RNAs displayed a more modest degree of U-tailing (12.96\% of 22G-RNA reads in IP samples vs. $5.18 \%$ in Input samples). We next assessed the uridylation of WAGO-4 22G-RNAs and found them to be uridylated to a similar extent as CSR-1a $(10.41 \%$ of 22 G-RNA reads in IP samples vs. $3.31 \%$ in Input samples). These uridylation differences of CSR-1b vs. CSR-1a and WAGO-4 22G-RNAs uncover another important distinction between the CSR-1 isoforms, and suggest that CSR-1a and WAGO-4 22G-RNAs are regulated differently than those in complex with CSR-1b. 
When examining the localization patterns of CSR-1 isoforms, we found that both CSR-1a and CSR-1b are present in at least two types of germ granules that are closely situated on the nuclear periphery: P granules and Z granules (Fig. 2B). Z granules have been implicated in transgeneral epigenetic inheritance, while P granules have been implicated in sRNA biogenesis and exoRNAi (78). WAGO-4 and the helicase ZNFX-1 were identified as the founding members of Z granules, and only a few other proteins have been identified in association with $Z$ granules so far $(65,76,78,79)$. Loss of either wago-4 or znfx-1 did not influence the localization of each other to Z granules $(65,76)$. However, loss of both isoforms of csr-1 disrupted ZNFX-1 foci in adult hermaphrodites, and was associated with a dissociation of PGL-1 from the nuclear periphery during oogenesis $(4,28,76)$.

Given the relationships we established between WAGO-4 and CSR-1a/b targets above, we wanted to determine if loss of wago-4 affected the association of either CSR-1 isoform with germ granules. To do this, we first quantified the association of GFP::CSR-1a, GFP::CSR-1b and GFP::WAGO-4 with the P and Z granule markers PGL-1::RFP and RFP::ZNFX-1, respectively, in L4 wild-type worms (Fig. 8E). We found that all three AGOs overlap with PGL-1 and ZNFX-1 pixels to a comparable extent. This implies that CSR-1a/b and WAGO-4 reside in both $\mathrm{P}$ and Z granules. We next performed the same quantification for each CSR-1 isoform in wago-4(tm1019) null mutants. While CSR-1a maintained an equivalent overlap with PGL-1 and ZNFX-1, CSR-1b displayed decreased overlap with PGL-1 and increased overlap with ZNFX-1. Loss of csr-1a led to no change in WAGO-4 overlap with either marker. These observations imply that loss of wago-4 shifts the balance of CSR-1b from $P$ granules to $Z$ granules, and reinforces the notion that CSR-1b and WAGO-4 act in concert to maintain germ granule homeostasis. In contrast, CSR-1a appears to function independently of WAGO4, and does not influence germ granule morphology or the balance between CSR-1b and WAGO-4. In sum, our data point to a broad role in germline constitutive gene regulation and germline homeostasis mediated via CSR-1b and WAGO-4, while CSR-1a and CSR-1b promote the expression of spermatogenesis transcripts. 
The AGOs ALG-3 and ALG-4 play an established role in spermatogenesis, and their functions have been linked to CSR-1 during sperm development $(14,23)$. Therefore, we aimed to assess how the CSR-1 isoforms intersect with the ALG-3, ALG-4 pathway. In addition, we have evaluated the expression patterns and sRNA binding partners of every AGO in a comprehensive study of all $C$. elegans AGOs (Seroussi, in preparation). This study uncovered a fourth WAGO with a spermatogenesis-specific expression profile: WAGO-10 (Fig. 9A). Consistent with its expression, WAGO-10 also associates with 22G-RNAs that target spermatogenesis transcripts (Seroussi, in preparation, and Fig. 10, Supp. Table S1-2). ALG-3, ALG-4, CSR-1a, and WAGO-10 therefore define a set of AGOs with spermatogenesis-specific germline expression.

The overlap in expression profiles and target gene sets (Fig. 9A, 10A, Supp. Table S2) suggested that these four AGOs could function redundantly to regulate spermatogenesis. Therefore, we built a quadruple null mutant for the spermatogenesis agos, and assessed male fertility. Double mutants of alg-3; alg-4 and csr-1 null mutants have previously been shown to exhibit a progressive loss of male fertility over several generations when cultured at the stressful temperature of $25^{\circ} \mathrm{C}(14,23)$. We also noticed that our csr-1a(tor159) allele showed a decrease in fertility at $25^{\circ} \mathrm{C}$ or after long-term culture at $20^{\circ} \mathrm{C}$ (Fig. 2D). Thus, we outcrossed each single spermatogenesis ago mutant and compared the brood size of each freshly outcrossed single mutant to the quadruple spermatogenesis ago mutant in the first generation experiencing the temperature shift. For each of the outcrossed single mutants, the brood size was comparable to wild-type worms (Fig. 9B). In sharp contrast, the quadruple ago mutant was nearly sterile in the first generation experiencing temperature shift. Further, we noticed that the quadruple spermatogenesis ago mutant produced only unfertilized oocytes, which is consistent with a defect in spermatogenesis. To determine whether the fertility defect was due to non-functional sperm, we crossed the quadruple spermatogenesis ago mutant hermaphrodites to wild-type males at $25^{\circ} \mathrm{C}$, and found that this rescued fertility to wild-type levels (Fig. 9C).

We were curious as to the cause of the spermatogenesis defect, so we took two approaches to broadly examine the phenotype. First, we DAPI stained L4 hermaphrodite wild-type vs. quadruple spermatogenesis ago mutants reared at $25^{\circ} \mathrm{C}$ to examine whether there were any developmental 
arrests or delays. Remarkably, we observed a block in spermatogenesis at the primary spermatocyte developmental stage in the quadruple spermatogenesis ago mutant (Fig. 9D). In fact, we did not detect mature spermatids in the majority of individuals examined from this strain. In parallel, we examined the number of germ granules (as measured by counting the number of ZNFX-1 and PGL-1 foci) present in quadruple ago mutants vs. wild-type worms at $25^{\circ} \mathrm{C}$, because germ granule dysfunction has been associated with a transgenerational loss of fertility in hermaphrodites (Fig. 9E). However, we did not detect any differences in the number of ZNFX-1 or PGL-1 foci, suggesting that defects in germ granule homeostasis were not at the root of the loss of fertility in the quadruple spermatogenesis ago mutant. Collectively, these data indicate that the spermatogenesis-specific AGOs, ALG-3, ALG-4, CSR-1a, and WAGO-10 cooperate to produce functional sperm and are essential for fertility.

\section{Interconnected AGO/sRNA modules target germline transcripts during spermatogenesis}

The ALG-3, ALG-4 26G-RNA pathway is thought to act upstream of CSR-1 (14). However, this model does not take into account the roles of other spermatogenesis-expressed AGOs, including the individual isoforms of CSR-1, WAGO-10, or WAGO-4. As a part of our comprehensive study of all $C$. elegans AGOs (Seroussi, in preparation), we performed IP/sRNA-seq experiments on ALG-3 and ALG4 to define their sets of 26G-RNA target transcripts, and WAGO-10 to define its 22G-RNA targets. Each of these AGOs targets protein coding genes (ALG-3: 2810; ALG-4 3246; WAGO-10: 786 genes) (Fig. 10A-B, Supp. Table S1-2). Given this abundance of sRNA targeting data, we now have an important opportunity to define the network of spermatogenic gene regulation in greater detail.

We first performed an unsupervised clustering analysis to visualize subsets of genes that were coordinately targeted by multiple AGOs (Fig. 10A). From this analysis, the overlap in WAGO-4 and CSR-1b targets discussed above is evident. Similarly, ALG-3 and ALG-4 show a high degree of overlap in their target transcripts, as was previously described $(14,23)$. WAGO-10 targets almost entirely overlap with CSR-1a, and with ALG-3 and ALG-4 targets, providing a possible explanation for the quadruple ago mutant fertility phenotype. 
We next compared the targets of each AGO to oogenic, spermatogenic and germline constitutive gene sets (29) (Fig. 10B, Supp. Table S2). We found that like CSR-1a, ALG-3 and ALG-4 targets are enriched, roughly equally, in germline constitutive and spermatogenic transcripts, while WAGO-10 targets are enriched in spermatogenic transcripts. Consistent with our analysis above, CSR$1 \mathrm{~b}$ targets are most enriched for germline constitutive genes. However, a comparable number of CSR$1 \mathrm{~b}$ targets are enriched in oogenesis and spermatogenesis genes, reflecting the different gene regulatory modules (WAGO-4 vs. CSR-1a) it participates in. WAGO-4 targets are enriched in germline constitutive and oogenic gene sets, and are conspicuously under-represented in spermatogenesis genes. From the clustering visualization (Fig. 10A), we can detect more specific subsets of sRNA target genes based on the combination of AGOs that target them (Fig. 10C, Supp. Table S2). This analysis reinforces our findings above. Gene sets targeted by WAGO-4 and any other AGO are overrepresented in germline constitutive and oogenic genes. Genes targeted by various combinations of ALG-3, ALG-4, WAGO-10, CSR-1a, and to a lesser extent CSR-1b (but excluding WAGO-4) are overrepresented in spermatogenic genes. Collectively, our findings implicate CSR-1b and WAGO-4 in a germline gene regulatory module that is likely to be important in both spermatogenesis and oogenesis (based on the nature of the genes targeted and the expression profiles of WAGO-4 and CSR-1b), while the CSR-1a, WAGO-10, ALG-3 and ALG-4 form a spermatogenesis gene regulatory module that is also likely to involve CSR-1b.

We homed in on genes involved in several important facets of spermatogenesis and spermiogenesis to understand how these different processes might be regulated. As anticipated, CSR$1 \mathrm{~b}$ and WAGO-4 appear to play broad roles in the germline that are important for both spermatogenesis and oogenesis. All Delta/Notch signaling factors involved in maintaining the germline stem cell niche (80) are targeted almost exclusively by CSR-1b and WAGO-4 22G-RNAs ( $g$ ld-2 is also a target of CSR1a, ALG-3, and ALG-4) (Fig. 10D, Supp. Table S2). The exception in this pathway is lag-2, the Delta ligand expressed in the distal tip cell, which is not targeted by any sRNA pathways described here. This is likely due to the fact that the distal tip cell is somatic. Similarly, the sex determination pathway (81) is extensively targeted by CSR-1b and WAGO-4 22G-RNAs (Fig. 10E, Supp. Table S2), as are many 
chromatin associated DNA and RNA binding proteins expressed in mature spermatozoa (Supp. Table S2) (66).

Sperm-specific functions are subject to regulation by the spermatogenesis AGOs. Genes involved in spermatogenesis—the differentiation of germ cells into sperm (22)—are predominantly targeted by ALG-3, ALG-4, CSR-1a and CSR-1b, and to a lesser extent WAGO-10 and WAGO-4 (Fig. 10F, Supp. Table S2). The Major Sperm Proteins (MSPs) are encoded by a group of about 30 highly homologous genes (along with about 20 pseudogenes) (82).. These small ( 15kDa), basic proteins are expressed late in spermatogenesis, and play roles in sperm structure, motility, and signaling (83). The msp genes are targeted by ALG-3, ALG-4, CSR-1a, and to a lesser extent, CSR-1b and WAGO-10 (Fig. 10G, Supp. Table S2). Notably WAGO-4 does not target any msp genes. Finally, the spermiogenesis, or sperm activation pathway, yields motile, fertilization competent spermatozoa from immature spermatids (83), and most components of this pathway are targeted by ALG-3, ALG-4, CSR1a, WAGO-10 am CSR-1b (Fig. 10H, Supp. Table S2). While these few examples only scratch the surface, they highlight the gene regulatory modules at play during spermatogenesis, and place CSR-1b at a critical intersection between the two major AGO networks.

\section{DISCUSSION}

Because it is the only essential AGO in C. elegans, exhibits several noteworthy developmental and molecular phenotypes, and regulates a large portion of the transcriptome, CSR-1 has been the focus of intense study since its initial characterization. Remarkably, CSR-1 is only conserved in clade III and $\mathrm{V}$ nematodes, yet in the only other nematode species in which its function has been studied, $C$. briggsae, it is also essential (84). The essential nature of CSR-1 points to important gene regulatory roles in gametogenesis and embryonic development, but until our study, it was unclear which isoforms of CSR-1 were responsible for these varied gene regulatory functions. Using CRISPR-Cas9 genome editing to GFP::3xFLAG tag each CSR-1 isoform, and mutant studies to understand the outcome of loss of each isoform on development, we have uncovered distinct expression patterns, sRNA binding partners, and gene regulatory functions for the two CSR-1 isoforms (Fig. 11). 


\section{CSR-1 isoforms regulate different facets of fertility}

Our previous studies using antibodies that recognize both CSR-1 isoforms failed to distinguish the expression patterns of CSR-1a and CSR-1b (4). By GFP::3xFLAG tagging the individual CSR-1 isoforms, we found that CSR-1b is expressed constitutively in the germline throughout all stages, and is present in somatic cells of the early embryo, due solely to maternal deposition. In contrast, CSR-1a expression in the germline is restricted to spermatogenesis. Moreover, CSR-1a is the only isoform expressed in the adult soma, where it is predominantly present in the intestine and spermatheca. These expression profiles alone shine a light on which CSR-1 isoform may be responsible for which phenotypes. Our analysis shows that CSR-1b alone carries out essential gene regulatory functions in the hermaphrodite germline and within embryos, where the sterile/lethal phenotype manifests.

Therefore, we conclude that $c s r-1 b$ is the essential isoform under "luxurious" lab culture conditions.

We were also surprised to find that the csr-1b rescue strain displayed lower fertility than the endogenously tagged GFP::3xFLAG::CSR-1b strain at $20^{\circ} \mathrm{C}$ (compare Fig. 4B to 4E), as both strains express only CSR-1b. This fertility defect appears to be due in large part to a late embryonic developmental arrest that occurs later than that of csr-1(tm892) null mutant embryos (Fig. S4F). This indicates that ectopic expression of CSR-1b from the rpl-28 promoter does not fully recapitulate wildtype CSR-1b expression, and suggests that the levels, developmental timing, and/or cells in which CSR-1b is expressed are subject to developmentally-important regulation. In particular, we speculate that based on the expression pattern of CSR-1b during embryogenesis, ectopic expression in somatic cells in late embryonic development could be deleterious for development.

Conversely, expression of csr-1a from the same rpl-28 promoter rescues the fertility defect of a csr-1(tm892) null mutant, suggesting that CSR-1a can perform at least some of the functions of CSR1b. Interestingly, when we sequenced sRNAs associated with CSR-1a in L4 hermaphrodites of the csr1a rescue strain, we found that CSR-1a binds to 22G-RNAs that target almost all of the CSR-1b targets (data not shown). This result is consistent with the fertility data, and reinforces the idea that CSR-1a can compensate at the molecular level for CSR-1b. Although we did not sequence sRNAs associated 
with ectopically expressed CSR-1a during oogenesis in the csr-1a rescue, we anticipate that CSR-1a binds to CSR-1b 22G-RNAs, resulting in fertility.

On its own, CSR-1a plays an important role in sperm-based fertility within hermaphrodites. Loss of csr-1a alone leads to a transgenerational loss of sperm-based fertility in hermaphrodites. CSR-1a also plays an overlapping role with ALG-3, ALG-4 and WAGO-10 in spermatogenesis; collectively these AGOs are required for generating mature sperm, and hence sperm-mediated fertility, at stressful temperatures. Because CSR-1b is also expressed throughout the germline during spermatogenesis, it is conceivable that it too is required for sperm-based fertility. Unfortunately, our data do not specifically address this issue. Comparing the transgenerational loss of fertility between csr-1a and csr-1 null mutants is fraught by the additional defects in oogenesis and embryogenesis caused by loss of $c s r-1 b$. Instead, to assess a role for CSR-1b in sperm-based fertility, we would need to examine male fertility in a transgenerational mating assay similar to Conine et al. (14) or rescue the csr-1(tm892) null mutant with a csr-1a transgene expressed from the csr-1 promoter. Selective degradation of CSR-1b during spermatogenesis via a degron approach (85) may also enable such an assessment. Related to this, any male-specific roles for either CSR-1 isoform should also be explored in the future. While many aspects of spermatogenesis are consistent between male and hermaphrodite $C$. elegans, there are several features that are sex-specific (86).

\section{CSR-1 isoforms associate with $\mathrm{P}$ and $\mathrm{Z}$ granules}

Our previous work showed that CSR-1 co-localizes with PGL-1 and is present within germ granules known as $\mathrm{P}$ granules (4). We initially speculated that CSR-1a might be the only isoform capable of localizing to $P$ granules due to its unique $R G G / R G$ rich $N$-terminus. $R G G / R G$ motifs have been implicated in RNA binding, recruiting RNA binding proteins, and inducing phase separation in vitro-all of which are functions relevant to germ granules (9). Loss of csr-1 has been shown to result in the mis-localization of $\mathrm{P}$ granules to the core cytoplasm of the germline, pointing to a role for CSR-1 in the maintenance and proper positioning of $P$ granules $(4,28)$. Remarkably, we observed that both 
CSR-1 isoforms overlap with $\mathrm{P}$ granule and Z granule markers to a similar extent in the L4 germline, using Mander's correlation. Examination of CSR-1b overlap with PGL-1 and ZNFX-1 during oogenesis yielded the same pattern (data not shown). There are multiple mechanisms by which proteins can associate with phase separated granules, including association with RNA and other proteins that

induce phase separation (9). The fact that CSR-1b, without an RGG/RG rich domain, and CSR-1a ${ }^{\text {[G91R] }}$ localize to germ granules supports the notion that RNA and/or other proteins enable the association of CSR-1 with these granules.

Prior to our findings, there were six known $Z$ granule components $(65,79,87)$, thus the identification of CSR-1 isoforms in association with ZNFX-1 is important. Although there are some limitations of our analysis to consider, including the facts that we were not able to visualize PGL-1, ZNFX-1, and CSR-1 in the same strains, and that our imaging represents a snapshot (and not live tracking) of development, there are several important implications. First, there may be an uncharacterized region of overlap between $\mathrm{Z}$ and $\mathrm{P}$ granules in the $\mathrm{L} 4$ germline where the CSR-1 isoforms reside, as opposed to CSR-1 isoforms existing as discrete pools within each granule. Second, it is possible that CSR-1 isoforms could move between the granules. Third, if the patterns we observed represent localization to distinct granules, CSR-1 isoforms could play different roles in the different granules, as $\mathrm{Z}$ granules have been linked to transgenerational epigenetic inheritance and $\mathrm{P}$ granules are associated with maternal inheritance and germ cell fate $(65,78)$.

CSR-1b and WAGO-4, a founding member of $Z$ granules, possess extensive overlap in their 22G-RNA targets. Thus, it may not be surprising that CSR-1b is present in Z granules, or that loss of wago-4 perturbs the distribution of CSR-1b between $\mathrm{P}$ and $\mathrm{Z}$ granules. The loss of wago-4 does not perturb the CSR-1a distribution, and notably, the overlap in CSR-1a and WAGO-4 targets is almost exclusively via targets shared with CSR-1b. Why and how germline genes are subject to regulation by different combinations of CSR-1a, CSR-1b, and WAGO-4 is currently unknown, but there is clearly a deeper molecular link that demands further study.

\section{How do CSR-1a's RG motifs contribute to its functions?}


Regarding the function of the RGG/RG motifs within the first exon of CSR-1a, we recovered several alleles that affected these motifs from the screen for proteasomal regulators. This could point to a role for arginine methylation in regulating CSR-1a. Arginine methylation is important for the recruitment of tudor domain proteins to AGO, for example, TudorSN and Piwi in Drosophila (6-8). In the worm, we hypothesize that tudor domain proteins, such as RSD-6 or EKL-1 (a known CSR-1 pathway component) $(4,15,63,88,89)$ may interact with CSR-1a in a methyl-arginine dependent manner.

We studied one of these alleles, $c s r-1 a^{[G 91 R]}$ in more detail. While mutation of a single RG motif in $c s r-1 a^{[G 91 R]}$ led to lower levels of CSR-1a expression, it did not perturb the formation of germ granules, nor did it prevent the association of CSR-1a with PGL-1 (Fig. S4). In addition, sequencing sRNAs associated with CSR-1a[G91R] showed a depletion for 22G-RNAs targeting approximately onehalf $(1279 / 2572)$ of the protein coding genes that are normally the targets of CSR-1a (data not shown). These genes are both germline and intestinal genes, suggesting that this particular RG motif could be important for gene regulation in both tissues. It is important to note that mutation of multiple RG residues may be necessary to perturb germ granules or cause substantial changes in sRNA association, thus a detailed mutagenesis study of these residues will be necessary in the future.

\section{CSR-1b associates with chromatin}

We also observed that CSR-1b associates with spermatid and oocyte nuclei and chromatin. These observations are consistent with a previous proteomic study that found CSR-1 peptides enriched on both oocyte and sperm chromatin (66), as well as microscopy and ChIP studies that point to an association of CSR-1 with chromatin $(4,10,14,18)$. These collective findings point to a low but consistent level of CSR-1b present in the nucleus and/or associated with germ cell chromatin, despite the fact that the bulk of CSR-1b protein is present within the cytoplasm and germ granules (4). This pool of CSR-1b can be passed from parent to progeny via sperm or oocyte chromatin and presents the possibility for epigenetically marking chromosomes, influencing chromatin landscapes, and reinforcing patterns of transcription in progeny $(14,19,90)$. CSR-1 has been implicated in transgenerational 
phenotypes, including Mrt and RNAa $(14,17,18)$, and this chromatin association could be but one of several molecular mechanisms by which these phenomena are executed.

\section{An extensive network of spermatogenesis sRNA pathways}

In addition to CSR-1a, we identified a second novel spermatogenesis-expressed AGO, WAGO10, and found that CSR-1a, WAGO-10, ALG-3 and ALG-4 that target spermatogenesis genes. This overlapping profile of gene targets for the spermatogenesis AGOs pointed to the potential for redundancy. While csr-1a, and alg-3; alg-4 mutants display a transgenerational loss of fertility at high temperatures (14), the quadruple spermatogenesis ago mutant displayed male-dependent infertility in a single generation at the stressful temperature, supporting that these AGOs function redundantly during spermatogenesis. Although we did not deeply examine the phenotype of quadruple spermatogenesis ago mutant germlines, we observed that germ granules appeared comparable in quantity to those of wild-type animals. However, germ cells were arrested in meiosis prior to differentiation into secondary spermatocytes, based on nuclear morphology. Further cell biological examination of this phenotype and characterization of any changes in gene regulation that result from loss of these four agos will be important next steps to understanding how these AGOs influence spermatogenesis.

We have stratified germline genes into different gene regulatory modules likely to be regulated by different AGOs. CSR-1b and WAGO-4 are constitutively expressed in the germline, and coordinately target germline constitutive and oogenic genes. CSR-1b is likely to play gene licensing rather than silencing roles $(4,14,18)$, while WAGO-4 appears to play a silencing role $(75)$. How these AGOs coordinate gene expression to maintain germline homeostasis and fertility remains unclear, and will undoubtedly be the focus of future studies.

CSR-1b also plays a role in regulating spermatogenic transcripts, this time alongside CSR-1a and the other spermatogenic AGOs. In this way, CSR-1b fills an important sRNA regulatory niche across all classes of germline genes. Perhaps this broad role for CSR-1b contributes to its essential nature. Exactly how CSR-1b regulates all of these different types of transcripts-at the cotranscriptional or post-transcriptional level-remains to be determined. There is some precedent for 
CSR-1's capacity to differentially regulate subsets of targets in the embryo $(10,11)$, but detailed molecular studies will need to be carried out in the germline to fully understand the scope of CSR-1b mechanisms.

The four spermatogenesis AGOs, ALG-3, ALG-4, CSR-1a, and WAGO-10, are expressed predominantly in the proximal germline, where they target mostly spermatogenic and some germline constitutive genes, but not oogenic genes. Perhaps not surprisingly, these AGOs target genes in all major pathways/components: spermatogenesis, spermiogenesis, and MSPs. Based on our current understanding of these pathways, it seems plausible that ALG-3 and ALG-4 26G-RNAs act in a primary capacity, while CSR-1a, WAGO-10, and to a lesser extent, CSR-1b 22G-RNAs act in a secondary capacity (14). ALG-3 and ALG-4 were previously implicated as licensing AGOs in conjunction with CSR-1 (14). Our mRNA-seq analysis in csr-1a(tor159) mutants supports this relationship: spermatogenic gene expression is decreased. It will be critical to examine gene expression changes in each single and combination spermatogenic ago mutant to determine the contribution of each AGO to fertility and gene regulation as well as to delineate functional distinctions and redundancies. Importantly, while these AGOs play an essential role in buffering the processes of spermatogenesis and spermiogenesis from stress to maintain fertility, their deposition into the residual body and depletion from maturing sperm indicates that they are unlikely to be direct couriers of transgenerational epigenetic inheritance.

\section{Somatic gene silencing roles for CSR-1a}

CSR-1a is the only CSR-1 isoform expressed in the adult soma, where it is required for repression of endogenous intestinal transcripts, including innate immunity and pathogen responsive genes. From our initial screen for proteasomal regulators, we isolated mutations affecting csr-1a and several known RNAi factors, including rde-1, rrf-1, and rsd-2. The screen was carried out using a repetitive array transgene (rpn-2::gfp), and $r d e-1, r r f-1$, and $r s d-2$ have all been implicated in exogenous and endogenous sRNA-mediated gene silencing (61-63). We therefore anticipated that CSR-1a may participate in somatic sRNA-mediated silencing of rpn-2::gfp. However, sRNA sequencing 
from $\operatorname{csr}-1 a^{\left[G 120^{*}\right]}$ and from CSR-1a IPs in the rpn-2::gfp strain showed no link between CSR-1a and sRNAs derived from the transgene, pointing to an indirect effect for CSR-1a in regulating the transgene. It is plausible that the other RNAi factors are involved in somatic transgene silencing, consistent with a previous report (91). However, at this point, it appears that CSR-1a affects the rpn-2::gfp transgene indirectly, possibly by affecting other regulators such as transcription factors, histone modifiers, or proteasome pathway components.

Interestingly, in an attempt to identify other somatic functions for CSR-1a, we also tested whether loss of csr-1a resulted in RNAi deficiency (Rde) (62). Null mutants of csr-1 have been shown to have limited capacity to silence germline transcripts, including pos-1 and cdk-1 (4). However, when we performed RNAi on $d p y-11$, an intestinal-expressed gene, we observed no difference between csr-1a mutants and wild-type worms (data not shown). While we were surprised that loss of csr-1a did not result in RNAi deficiency, our systematic study of $C$. elegans AGOs points to several other AGOs that are expressed in the intestine and have been previously implicated in RNAi, including the secondary sRNA binding AGOs, SAGO-1 and SAGO-2 (3) (Seroussi, in preparation). One particular interesting candidate is SAGO-2, which has recently been shown to interact with CSR-1a by mass spectrometry (92). Thus, it is possible that these AGOs could function redundantly with CSR-1a to execute exogenous RNAi in this tissue.

Loss of csr-1a led to the increased expression of many somatic genes in the soma. This suggests that CSR-1a silences the expression of its targets in the intestine, and possibly other somatic tissues. Our gene expression analysis hinted that CSR-1a could play a role in pathogen and/or stress responses within the intestine, as genes related to defense responses, interspecies interaction and the immune system were upregulated in csr-1a(tor159) mutants. Despite this observation, we were still somewhat surprised to find that loss of csr-1a was beneficial to worm survival from the bacterial pathogen Pseudomonas aeruginosa. We reason that upregulation of such genes could enable the intestine to be "primed" for encounters with pathogens or other environmental stressors. It will be important to test the response of csr-1a(tor159) mutants to a full array of pathogens and environmental stresses in the future, to determine if this is a generalized or specialized response. 


\section{Slicer activity of CSR-1 isoforms}

Our studies uncover many new dimensions of CSR-1 biology, however they do not address whether Slicer function is important for both CSR-1 isoforms and in what tissues or gene regulatory contexts. Both isoforms possess the catalytic tetrad of residues within the PIWI domain that are required for Slicer activity, and CSR-1 is one of few AGOs in the worm for which Slicer activity has been studied in vitro and in vivo $(11,20,21)$. Slicer activity has been implicated in fine tuning maternally deposited transcripts with high 22G-RNA density in the embryo (11). This function can be attributed to CSR-1b based on expression of the isoforms alone. Histone mRNA maturation is another activity for which Slicer activity is anticipated to be required. As both CSR-1 isoforms associate with 22G-RNAs that target half of all histone transcripts, Slicer activity may be important for both isoforms in this context. Clearly, detailed tissue-specific studies will be important to delineate the roles of Slicer activity for each isoform throughout development.

\section{Summary: A tale of two Argonautes}

Our studies disentangle a significant amount of complexity surrounding the essential $C$. elegans AGO, CSR-1, and generate a myriad of additional questions surrounding the tissue-specific functions of CSR-1 and other AGOs. Newly developed means of expressing and degrading or inactivating proteins in a tissue specific manner will enable us to understand the roles of each CSR-1 isoform at a finer resolution, in specific cells and tissues (85).

In other Caenorhabditis nematodes, particularly male/female mating species, csr-1 not only has multiple isoforms, but possesses duplicated gene copies $(84,93)$. This expansion has been proposed to serve as a defense mechanism against viruses and other invasive nucleic acid transmitted between the members of obligatory mating species (93). We have shown that CSR-1a buffers the effects of temperature stress during spermatogenesis to maintain fertility and is involved in gene silencing in the intestine, a tissue that directly interfaces with the environment. Given its roles in $C$. elegans, it is 
possible that these additional copies of csr-1 have further specialized to enable other Caenorhabditis species to navigate an array of stresses encountered in their natural environment.

Importantly, CSR-1 is not the only Argonaute expressed as multiple isoforms. Several other AGOs, including ALG-1, ALG-2, ERGO-1, and PPW-1, also possess this capacity in C. elegans, but isoform-specific functions of these proteins have not been examined. Our work highlights the importance of dissecting each of these AGO isoforms' roles to unravel how complex tissue- and developmental stage-specific sRNA networks safeguard the genome, germline, and soma.

\section{DATA AVAILABILITY}

Small RNA-seq and poly(A) selected mRNA-seq data generated in this study are available under the accession number GEO: GSE154678. Germline constitutive, oogenic and spermatogenic gene lists were defined as in (29) available from GEO under accession number GSE57109. RdRP mutant small RNA data are from (71) [GSE66344]. alg-3, alg-4 mutant sRNA data are from (23) [GSE49672]. WAGO-1 IP/sRNA-seq data was obtained from (15) available under accession number [GSE18231]. The CSR-1 IP/sRNA-seq data from males was obtained from (14), accession number [GSE49672], while the hermaphrodite data was obtained from (4), accession number [GSE18167]. The custom scripts used to generate the processed data are available at www.github.com/ClaycombLab/Charlesworth_2020.

\section{SUPPLEMENTARY DATA}

Supplementary data (S1-S5) and tables (Table S1-S3) are presented in separate files.

\section{FUNDING}

This work was funded by Canadian Institutes of Health Research Grants to JMC (PJT-156083 and PJG-175378). JMC is supported by a CRC Tier II in Small RNA Biology. MSR is supported by a CIHRCGSM fellowship. Some strains were provided by the CGC, which is funded by NIH Office of Research Infrastructure Programs (P40 OD010440). 


\section{CONFLICT OF INTEREST}

The authors declare no competing interests.

\section{ACKNOWLEDGEMENTS}

The authors thank Drs. John Calarco and Arneet Saltzman, and members of the Claycomb lab for critical reading and constructive comments on this manuscript. We thank Dr. Carolyn Phillips and her lab for thoughtful discussions about the CSR-1 isoforms. Thanks to the labs of Drs. Aaron Reinke and Thomas Hurd for use of microscopes, and to WormBase and The Caenorhabditis Genetics Center. We are also grateful to the Toronto Area Worm Community for reagents, protocols, and discussions.

Authors' contributions: AGC, NJL, US, MSR, MJA, JRW, AES, RXL, RIM, and AJD performed the experiments. AGC, MSR, US, and AJD performed computational analyses. JMC wrote the manuscript, with the assistance of AGC, NJL, and RIM. JMC, AGC, US, NJL, MSR, AES, RIM, and AJD devised the figures. 


\section{REFERENCES}

1. Youngman,E.M. and Claycomb,J.M. (2014) From early lessons to new frontiers: the worm as a treasure trove of small RNA biology. Front Genet 5, 416.

2. Meister,G. (2013) Argonaute proteins: functional insights and emerging roles. Nat Rev Genet 14, 447-459.

3. Yigit,E., Batista,P.J., Bei,Y., Pang,K.M., Chen,C.C., Tolia,N.H., Joshua-Tor,L., Mitani,S., Simard,M.J. and Mello,C.C. (2006) Analysis of the C. elegans Argonaute family reveals that distinct Argonautes act sequentially during RNAi. Cell 127, 747-757.

4. Claycomb,J.M., Batista,P.J., Pang,K.M., Gu,W., Vasale,J.J., van Wolfswinkel,J.C., Chaves,D.A., Shirayama,M., Mitani,S., Ketting,R.F., Conte,D.J. and Mello,C.C. (2009) The Argonaute CSR-1 and its 22G-RNA cofactors are required for holocentric chromosome segregation. Cell 139, 123134.

5. Thandapani,P., O'Connor,T.R., Bailey,T.L. and Richard,S. (2013) Defining the RGG/RG motif. $\mathrm{Mol}$ Cell 50, 613-623.

6. Kirino,Y., Kim,N., de Planell-Saguer,M., Khandros,E., Chiorean,S., Klein,P.S., Rigoutsos,I., Jongens,T.A. and Mourelatos,Z. (2009) Arginine methylation of Piwi proteins catalysed by dPRMT5 is required for Ago3 and Aub stability. Nat Cell Biol 11, 652-658.

7. Liu,K., Chen,C., Guo,Y., Lam,R., Bian,C., Xu,C., Zhao,D.Y., Jin,J., MacKenzie,F., Pawson,T. and Min,J. (2010) Structural basis for recognition of arginine methylated Piwi proteins by the extended Tudor domain. Proc Natl Acad Sci U S A 107, 18398-18403.

8. Vagin,V.V., Wohlschlegel,J., Qu,J., Jonsson,Z., Huang,X., Chuma,S., Girard,A., Sachidanandam,R., Hannon,G.J. and Aravin,A.A. (2009) Proteomic analysis of murine Piwi proteins reveals a role for arginine methylation in specifying interaction with Tudor family members. Genes Dev 23, 1749-1762.

9. Chong,P.A., Vernon,R.M. and Forman-Kay,J.D. (2018) RGG/RG Motif Regions in RNA Binding and Phase Separation. J Mol Biol 430, 4650-4665.

10. Avgousti,D.C., Palani,S., Sherman,Y. and Grishok,A. (2012) CSR-1 RNAi pathway positively regulates histone expression in C. elegans. EMBO J 31, 3821-3832.

11. Gerson-Gurwitz,A., Wang,S., Sathe,S., Green,R., Yeo,G.W., Oegema,K. and Desai,A. (2016) A Small RNA-Catalytic Argonaute Pathway Tunes Germline Transcript Levels to Ensure Embryonic Divisions. Cell 165, 396-409.

12. Wedeles,C.J., Wu,M.Z. and Claycomb,J.M. (2013) A multitasking Argonaute: exploring the many facets of C. elegans CSR-1. Chromosome Res 21, 573-586.

13. Quarato,P., Singh,M., Cornes,E., Li,B., Bourdon,L., Mueller,F., Didier,C. and Cecere,G. (2021) Germline inherited small RNAs facilitate the clearance of untranslated maternal mRNAs in C. elegans embryos. Nat Commun 12, 1441.

14. Conine,C.C., Moresco,J.J., Gu,W., Shirayama,M., Conte,D.J., Yates,J.R. and Mello,C.C. (2013) Argonautes Promote Male Fertility and Provide a Paternal Memory of Germline Gene Expression in C. elegans. Cell 155, 1532-1544.

15. Gu,W., Shirayama,M., Conte,D.J., Vasale,J., Batista,P.J., Claycomb,J.M., Moresco,J.J., Youngman,E.M., Keys,J., Stoltz,M.J., Chen,C.C., Chaves,D.A., Duan,S., Kasschau,K.D., Fahlgren,N., Yates,J.R., Mitani,S., Carrington,J.C. and Mello,C.C. (2009) Distinct argonautemediated 22G-RNA pathways direct genome surveillance in the C. elegans germline. Mol Cell 36, 231-244.

16. Cecere,G., Hoersch,S., O'Keeffe,S., Sachidanandam,R. and Grishok,A. (2014) Global effects of the CSR-1 RNA interference pathway on the transcriptional landscape. Nat Struct Mol Biol 21, 358-365.

17. Seth,M., Shirayama,M., Gu,W., Ishidate,T., Conte,D. and Mello,C.C. (2013) The C. elegans CSR1 argonaute pathway counteracts epigenetic silencing to promote germline gene expression. Dev Cell 27, 656-663. 
18. Wedeles,C.J., Wu,M.Z. and Claycomb,J.M. (2013) Protection of germline gene expression by the C. elegans Argonaute CSR-1. Dev Cell 27, 664-671.

19. Gushchanskaia,E.S., Esse,R., Ma,Q., Lau,N.C. and Grishok,A. (2019) Interplay between small RNA pathways shapes chromatin landscapes in C. elegans. Nucleic Acids Res 47, 5603-5616.

20. Aoki,K., Moriguchi,H., Yoshioka,T., Okawa,K. and Tabara,H. (2007) In vitro analyses of the production and activity of secondary small interfering RNAs in C. elegans. EMBO J 26, 50075019.

21. Fassnacht,C., Tocchini,C., Kumari,P., Gaidatzis,D., Stadler,M.B. and Ciosk,R. (2018) The CSR-1 endogenous RNAi pathway ensures accurate transcriptional reprogramming during the oocyte-toembryo transition in Caenorhabditis elegans. PLoS Genet 14, e1007252.

22. L'Hernault,S.W. (2006) Spermatogenesis. WormBook 1-14.

23. Conine,C.C., Batista,P.J., Gu,W., Claycomb,J.M., Chaves,D.A., Shirayama,M. and Mello,C.C. (2010) Argonautes ALG-3 and ALG-4 are required for spermatogenesis-specific 26G-RNAs and thermotolerant sperm in Caenorhabditis elegans. Proc Natl Acad Sci U S A 107, 3588-3593.

24. Chaves,D.A., Dai,H., Li,L., Moresco,J.J., Oh,M.E., Conte,D., Yates,J.R., Mello,C.C. and Gu,W. (2021) The RNA phosphatase PIR-1 regulates endogenous small RNA pathways in C. elegans. Mol Cell 81, 546-557.e5.

25. Han,T., Manoharan,A.P., Harkins,T.T., Bouffard,P., Fitzpatrick,C., Chu,D.S., Thierry-Mieg,D., Thierry-Mieg,J. and Kim,J.K. (2009) 26G endo-siRNAs regulate spermatogenic and zygotic gene expression in Caenorhabditis elegans. Proc Natl Acad Sci U S A 106, 18674-18679.

26. Gent,J.I., Schvarzstein,M., Villeneuve,A.M., Gu,S.G., Jantsch,V., Fire,A.Z. and Baudrimont,A. (2009) A Caenorhabditis elegans RNA-directed RNA polymerase in sperm development and endogenous RNA interference. Genetics 183, 1297-1314.

27. Vasale,J.J., Gu,W., Thivierge,C., Batista,P.J., Claycomb,J.M., Youngman,E.M., Duchaine,T.F., Mello,C.C. and Conte,D.J. (2010) Sequential rounds of RNA-dependent RNA transcription drive endogenous small-RNA biogenesis in the ERGO-1/Argonaute pathway. Proc Natl Acad Sci U S A 107, 3582-3587.

28. Campbell,A.C. and Updike,D.L. (2015) CSR-1 and P granules suppress sperm-specific transcription in the C. elegans germline. Development 142, 1745-1755.

29. Ortiz,M.A., Noble,D., Sorokin,E.P. and Kimble,J. (2014) A new dataset of spermatogenic vs. oogenic transcriptomes in the nematode Caenorhabditis elegans. G3 (Bethesda) 4, 1765-1772.

30. Brenner,S. (1974) The genetics of Caenorhabditis elegans. Genetics 77, 71-94.

31. Schnabel,H. and Schnabel,R. (1990) An Organ-Specific Differentiation Gene, pha-1, from Caenorhabditis elegans. Science 250, 686-688.

32. Dickinson,D.J., Ward,J.D., Reiner,D.J. and Goldstein,B. (2013) Engineering the Caenorhabditis elegans genome using Cas9-triggered homologous recombination. Nat Methods 10, 1028-1034.

33. Paix,A., Wang,Y., Smith,H.E., Lee,C.Y., Calidas,D., Lu,T., Smith,J., Schmidt,H., Krause,M.W. and Seydoux,G. (2014) Scalable and versatile genome editing using linear DNAs with microhomology to Cas9 Sites in Caenorhabditis elegans. Genetics 198, 1347-1356.

34. Ward,J.D. (2015) Rapid and precise engineering of the Caenorhabditis elegans genome with lethal mutation co-conversion and inactivation of NHEJ repair. Genetics 199, 363-377.

35. Arribere,J.A., Bell,R.T., Fu,B.X., Artiles,K.L., Hartman,P.S. and Fire,A.Z. (2014) Efficient markerfree recovery of custom genetic modifications with CRISPR/Cas9 in Caenorhabditis elegans. Genetics 198, 837-846.

36. Dickinson,D.J., Pani,A.M., Heppert,J.K., Higgins,C.D. and Goldstein,B. (2015) Streamlined Genome Engineering with a Self-Excising Drug Selection Cassette. Genetics 200, 1035-1049.

37. Ouyang,J.P.T., Folkmann,A., Bernard,L., Lee,C.Y., Seroussi,U., Charlesworth,A.G., Claycomb,J.M. and Seydoux,G. (2019) P Granules Protect RNA Interference Genes from Silencing by piRNAs. Dev Cell 50, 716-728.e6.

38. Lev,I., Toker,I.A., Mor,Y., Nitzan,A., Weintraub,G., Antonova,O., Bhonkar,O., Ben Shushan,I., Seroussi,U., Claycomb,J.M., Anava,S., Gingold,H., Zaidel-Bar,R. and Rechavi,O. (2019) Germ Granules Govern Small RNA Inheritance. Curr Biol 29, 2880-2891.e4.

39. Gibson,D.G. (2009) Synthesis of DNA fragments in yeast by one-step assembly of overlapping oligonucleotides. Nucleic Acids Res 37, 6984-6990. 
40. Lehrbach,N.J. and Ruvkun,G. (2016) Proteasome dysfunction triggers activation of SKN-1A/Nrf1 by the aspartic protease DDI-1. Elife 5, e17721.

41. Frøkjær-Jensen,C., Davis,M.W., Sarov,M., Taylor,J., Flibotte,S., LaBella,M., Pozniakovsky,A., Moerman,D.G. and Jorgensen,E.M. (2014) Random and targeted transgene insertion in Caenorhabditis elegans using a modified Mos1 transposon. Nat Methods 11, 529-534.

42. Hunt-Newbury,R., Viveiros,R., Johnsen,R., Mah,A., Anastas,D., Fang,L., Halfnight,E., Lee,D., Lin,J., Lorch,A., McKay,S., Okada,H.M., Pan,J., Schulz,A.K., Tu,D., Wong,K., Zhao,Z., Alexeyenko,A., Burglin,T., Sonnhammer,E., Schnabel,R., Jones,S.J., Marra,M.A., Baillie,D.L. and Moerman,D.G. (2007) High-throughput in vivo analysis of gene expression in Caenorhabditis elegans. PLoS Biol 5, e237.

43. Minevich,G., Park,D.S., Blankenberg,D., Poole,R.J. and Hobert,O. (2012) CloudMap: a cloudbased pipeline for analysis of mutant genome sequences. Genetics 192, 1249-1269.

44. Schindelin,J., Arganda-Carreras,I., Frise,E., Kaynig,V., Longair,M., Pietzsch,T., Preibisch,S., Rueden,C., Saalfeld,S., Schmid,B., Tinevez,J.Y., White,D.J., Hartenstein,V., Eliceiri,K., Tomancak,P. and Cardona,A. (2012) Fiji: an open-source platform for biological-image analysis. Nat Methods 9, 676-682.

45. Bolte,S. and Cordelières,F.P. (2006) A guided tour into subcellular colocalization analysis in light microscopy. J Microsc 224, 213-232.

46. Schneider,C.A., Rasband,W.S. and Eliceiri,K.W. (2012) NIH Image to ImageJ: 25 years of image analysis. Nat Methods 9, 671-675.

47. Fox,J. and Weisberg,S. (2019) Sage.

48. Wickham,H. (2016) Springer-Verlag New York.

49. Tan,M.W., Rahme,L.G., Sternberg,J.A., Tompkins,R.G. and Ausubel,F.M. (1999) Pseudomonas aeruginosa killing of Caenorhabditis elegans used to identify P. aeruginosa virulence factors. Proc Natl Acad Sci U S A 96, 2408-2413.

50. Martin,M. (2011) Cutadapt removes adapter sequences from high-throughput sequencing reads Bioinformatics in action 17, 10-12.

51. Dobin,A., Davis,C.A., Schlesinger,F., Drenkow,J., Zaleski,C., Jha,S., Batut,P., Chaisson,M. and Gingeras,T.R. (2013) STAR: ultrafast universal RNA-seq aligner. Bioinformatics 29, 15-21.

52. Smith,R.N., Aleksic,J., Butano,D., Carr,A., Contrino,S., Hu,F., Lyne,M., Lyne,R., Kalderimis,A., Rutherford,K., Stepan,R., Sullivan,J., Wakeling,M., Watkins,X. and Micklem,G. (2012) InterMine: a flexible data warehouse system for the integration and analysis of heterogeneous biological data. Bioinformatics 28, 3163-3165.

53. Kalderimis,A., Lyne,R., Butano,D., Contrino,S., Lyne,M., Heimbach,J., Hu,F., Smith,R., Stěpán,R., Sullivan,J. and Micklem,G. (2014) InterMine: extensive web services for modern biology. Nucleic Acids Res 42, W468-72.

54. Yang,W., Dierking,K. and Schulenburg,H. (2016) WormExp: a web-based application for a Caenorhabditis elegans-specific gene expression enrichment analysis. Bioinformatics 32, 943945.

55. Angeles-Albores,D., Lee,R., Chan,J. and Sternberg,P. (2018) Two new functions in the WormBase Enrichment Suite. MicroPubl Biol 2018,

56. Love,M.I., Huber,W. and Anders,S. (2014) Moderated estimation of fold change and dispersion for RNA-seq data with DESeq2. Genome Biol 15, 550.

57. Benson,D.A., Cavanaugh,M., Clark,K., Karsch-Mizrachi,I., Lipman,D.J., Ostell,J. and Sayers,E.W. (2017) GenBank. Nucleic Acids Res 45, D37-D42.

58. Edgar,R.C. (2004) MUSCLE: multiple sequence alignment with high accuracy and high throughput. Nucleic Acids Res 32, 1792-1797.

59. Gouy,M., Guindon,S. and Gascuel,O. (2010) SeaView version 4: A multiplatform graphical user interface for sequence alignment and phylogenetic tree building. Mol Biol Evol 27, 221-224.

60. Bard,J.A.M., Goodall,E.A., Greene,E.R., Jonsson,E., Dong,K.C. and Martin,A. (2018) Structure and Function of the 26S Proteasome. Annu Rev Biochem 87, 697-724.

61. Sijen,T., Fleenor,J., Simmer,F., Thijssen,K.L., Parrish,S., Timmons,L., Plasterk,R.H. and Fire,A. (2001) On the role of RNA amplification in dsRNA-triggered gene silencing. Cell 107, 465-476. 
62. Tabara,H., Sarkissian,M., Kelly,W.G., Fleenor,J., Grishok,A., Timmons,L., Fire,A. and Mello,C.C. (1999) The rde-1 gene, RNA interference, and transposon silencing in C. elegans. Cell 99, 123132.

63. Tijsterman,M., May,R.C., Simmer,F., Okihara,K.L. and Plasterk,R.H. (2004) Genes required for systemic RNA interference in Caenorhabditis elegans. Curr Biol 14, 111-116.

64. Kim,H., Ishidate,T., Ghanta,K.S., Seth,M., Conte,D., Shirayama,M. and Mello,C.C. (2014) A coCRISPR strategy for efficient genome editing in Caenorhabditis elegans. Genetics 197, 10691080.

65. Wan,G., Fields,B.D., Spracklin,G., Shukla,A., Phillips,C.M. and Kennedy,S. (2018) Spatiotemporal regulation of liquid-like condensates in epigenetic inheritance. Nature 557, 679-683.

66. Chu,D.S., Liu,H., Nix,P., Wu,T.F., Ralston,E.J., Yates,J.R. and Meyer,B.J. (2006) Sperm chromatin proteomics identifies evolutionarily conserved fertility factors. Nature 443, 101-105.

67. Fukushige,T., Hawkins,M.G. and McGhee,J.D. (1998) The GATA-factor elt-2 is essential for formation of the Caenorhabditis elegans intestine. Dev Biol 198, 286-302.

68. Kudron,M.M., Victorsen,A., Gevirtzman,L., Hillier,L.W., Fisher,W.W., Vafeados,D., Kirkey,M., Hammonds,A.S., Gersch,J., Ammouri,H., Wall,M.L., Moran,J., Steffen,D., Szynkarek,M., Seabrook-Sturgis,S., Jameel,N., Kadaba,M., Patton,J., Terrell,R., Corson,M., Durham,T.J., Park,S., Samanta,S., Han,M., Xu,J., Yan,K.K., Celniker,S.E., White,K.P., Ma,L., Gerstein,M., Reinke,V. and Waterston,R.H. (2018) The ModERN Resource: Genome-Wide Binding Profiles for Hundreds of Genetics 208, 937-949.

69. Kasper,D.M., Wang,G., Gardner,K.E., Johnstone,T.G. and Reinke,V. (2014) The C. elegans SNAPc component SNPC-4 coats piRNA domains and is globally required for piRNA abundance. Dev Cell 31, 145-158.

70. Harrison,M.M., Ceol,C.J., Lu,X. and Horvitz,H.R. (2006) Some C. elegans class B synthetic multivulva proteins encode a conserved LIN-35 Rb-containing complex distinct from a NuRD-like complex. Proc Natl Acad Sci U S A 103, 16782-16787.

71. Sapetschnig,A., Sarkies,P., Lehrbach,N.J. and Miska,E.A. (2015) Tertiary siRNAs mediate paramutation in C. elegans. PLoS Genet 11, e1005078.

72. Davis,G.M., Tu,S., Anderson,J.W., Colson,R.N., Gunzburg,M.J., Francisco,M.A., Ray,D., Shrubsole,S.P., Sobotka,J.A., Seroussi,U., Lao,R.X., Maity,T., Wu,M.Z., McJunkin,K., Morris,Q.D., Hughes, T.R., Wilce,J.A., Claycomb,J.M., Weng,Z. and Boag,P.R. (2018) The TRIMNHL protein NHL-2 is a co-factor in the nuclear and somatic RNAi pathways in Elife 7,

73. Keall,R., Whitelaw,S., Pettitt,J. and Müller,B. (2007) Histone gene expression and histone mRNA 3 ' end structure in Caenorhabditis elegans. BMC Mol Biol 8, 51.

74. Mahajan-Miklos,S., Tan,M.W., Rahme,L.G. and Ausubel,F.M. (1999) Molecular mechanisms of bacterial virulence elucidated using a Pseudomonas aeruginosa-Caenorhabditis elegans pathogenesis model. Cell 96, 47-56.

75. Xu,F., Feng,X., Chen,X., Weng,C., Yan,Q., Xu,T., Hong,M. and Guang,S. (2018) A Cytoplasmic Argonaute Protein Promotes the Inheritance of RNAi. Cell Rep 23, 2482-2494.

76. Ishidate,T., Ozturk,A.R., Durning,D.J., Sharma,R., Shen,E.Z., Chen,H., Seth,M., Shirayama,M. and Mello,C.C. (2018) ZNFX-1 Functions within Perinuclear Nuage to Balance Epigenetic Signals. Mol Cell 70, 639-649.e6.

77. van Wolfswinkel,J.C., Claycomb,J.M., Batista,P.J., Mello,C.C., Berezikov,E. and Ketting,R.F. (2009) CDE-1 affects chromosome segregation through uridylation of CSR-1-bound siRNAs. Cell 139, $135-148$.

78. Sundby,A.E., Molnar,R.I. and Claycomb,J.M. (2021) Connecting the Dots: Linking Caenorhabditis elegans Small RNA Pathways and Germ Granules. Trends Cell Biol 31, 387-401.

79. Wan,G., Bajaj,L., Fields,B., Dodson,A.E., Pagano,D., Fei,Y. and Kennedy,S. (2021) ZSP-1 is a Z granule surface protein required for $Z$ granule fluidity and germline immortality in Caenorhabditis elegans. EMBO J 40, e105612.

80. White-Cooper,H. and Bausek,N. (2010) Evolution and spermatogenesis. Philos Trans R Soc Lond B Biol Sci 365, 1465-1480.

81. Ellis,R. and Schedl,T. (2007) Sex determination in the germ line. WormBook 1-13. 
82. Scott,A.L., Dinman,J., Sussman,D.J. and Ward,S. (1989) Major sperm protein and actin genes in free-living and parasitic nematodes. Parasitology 98 Pt 3, 471-478.

83. Smith,H.E. (2014) Nematode sperm motility. WormBook 1-15.

84. Tu,S., Wu,M.Z., Wang,J., Cutter,A.D., Weng,Z. and Claycomb,J.M. (2015) Comparative functional characterization of the CSR-1 22G-RNA pathway in Caenorhabditis nematodes. Nucleic Acids Res 43, 208-224.

85. Martinez,M.A.Q., Kinney,B.A., Medwig-Kinney,T.N., Ashley,G., Ragle,J.M., Johnson,L., Aguilera,J., Hammell,C.M., Ward,J.D. and Matus,D.Q. (2020) Rapid Degradation of Caenorhabditis elegans Proteins at Single-Cell Resolution with a Synthetic Auxin G3 (Bethesda) 10, 267-280.

86. L'Hernault,S.W. (2009) The genetics and cell biology of spermatogenesis in the nematode C. elegans. Mol Cell Endocrinol 306, 59-65.

87. Placentino,M., de Jesus Domingues,A.M., Schreier,J., Dietz,S., Hellmann,S., de Albuquerque,B.F., Butter,F. and Ketting,R.F. (2021) Intrinsically disordered protein PID-2 modulates $Z$ granules and is required for heritable piRNA-induced silencing in the Caenorhabditis elegans embryo. EMBO J 40, e105280.

88. Zhang,C., Montgomery,T.A., Fischer,S.E., Garcia,S.M., Riedel,C.G., Fahlgren,N., Sullivan,C.M., Carrington,J.C. and Ruvkun,G. (2012) The Caenorhabditis elegans RDE-10/RDE-11 complex regulates RNAi by promoting secondary siRNA amplification. Curr Biol 22, 881-890.

89. Sakaguchi,A., Sarkies,P., Simon,M., Doebley,A.L., Goldstein,L.D., Hedges,A., Ikegami,K., Alvares,S.M., Yang,L., LaRocque,J.R., Hall,J., Miska,E.A. and Ahmed,S. (2014) Caenorhabditis elegans RSD-2 and RSD-6 promote germ cell immortality by maintaining small interfering RNA populations. Proc Natl Acad Sci U S A 111, E4323-31.

90. She,X., Xu,X., Fedotov,A., Kelly,W.G. and Maine,E.M. (2009) Regulation of heterochromatin assembly on unpaired chromosomes during Caenorhabditis elegans meiosis by components of a small RNA-mediated pathway. PLoS Genet 5, e1000624.

91. Grishok,A., Sinskey,J.L. and Sharp,P.A. (2005) Transcriptional silencing of a transgene by RNAi in the soma of C. elegans. Genes Dev 19, 683-696.

92. Barucci,G., Cornes,E., Singh,M., Li,B., Ugolini,M., Samolygo,A., Didier,C., Dingli,F., Loew,D., Quarato,P. and Cecere,G. (2020) Small-RNA-mediated transgenerational silencing of histone genes impairs fertility in piRNA mutants. Nat Cell Biol 22, 235-245.

93. Shi,Z., Montgomery,T.A., Qi,Y. and Ruvkun,G. (2013) High-throughput sequencing reveals extraordinary fluidity of miRNA, piRNA, and siRNA pathways in nematodes. Genome Res 23, 497-508. 


\section{FIGURE LEGENDS}

Figure 1. CSR-1a and CSR-1b isoforms are differentially expressed throughout development.

A) Schematic representation of CSR-1 isoforms. Black boxes are exons, gray boxes are 3'UTRs. B) Multiple sequence alignment of the $\mathrm{N}$-terminal region of $c s r-1$ for nine Caenorhabditis species. Arrows indicate $C$. elegans amino acid positions mutated as a result of the forward genetic screen (as shown in Fig. S1B-C). The asterisk indicates the first amino acid of CSR-1b in C. elegans. C) Schematic representations of all csr-1 mutant strains, endogenously tagged strains and single copy transgene insertion strains used in this study. D) Western blot using GFP antibodies to detect GFP::3xFLAG::CSR-1, GFP::3xFLAG::CSR-1b, and GFP::3xFLAG::CSR-1a expression in each life stage (larval stages 1 to 4 (L1-L4), young adult (YA), gravid adult (GA) and embryo (EMB) stages). E) Fluorescence micrographs of whole animals expressing endogenously tagged CSR-1 isoforms during each life stage (L1-L4, YA and GA). Scale bar, 50um.

Figure 2. CSR-1a and CSR-1b isoforms are differentially expressed in the germline.

A) Fluorescence micrographs of animals in L4 and gravid adult (GA) stages expressing GFP::3xFLAG::CSR-1, GFP::3xFLAG::CSR-1b and GFP::3xFLAG::CSR-1a in the germlines. Scale bar, $50 \mu \mathrm{m}$. B) Fluorescence micrographs of $L 4$ and young adult (YA) germlines expressing GFP::3xFLAG::CSR-1b and GFP::3xFLAG::CSR-1a (green), along with either PGL-1::mRGFP or HA::TagRFP::ZNFX-1 (red). Top panels show the pachytene region of each germline, and bottom panels show a single nucleus. Note that CSR-1a is not expressed in the YA stage. Scale bar, 10 $\mu \mathrm{m}$ in top images, $1 \mu \mathrm{m}$ in bottom images. C) Fluorescence micrographs of residual bodies (outlined in yellow) and spermatocytes (top) spermatids(bottom) (both outlined in white) from animals expressing GFP::3xFLAG::CSR-1, GFP::3xFLAG::CSR-1b and GFP::3xFLAG::CSR-1a and stained with DAPI (blue in Merge), compared to wild-type (N2) animals. Scale bar, 5um. D) Fluorescence micrographs showing localization of endogenously tagged CSR-1 isoforms in oocytes (top) and time lapse of endogenously tagged CSR-1 localization in the -1 oocyte over the course of five minutes (bottom). Scale bar, $25 \mu \mathrm{m}$. E) Fluorescence micrographs showing expression of endogenously tagged CSR-1 
isoforms and total CSR-1 (reflecting CSR-1b expression) in 2-cell embryos prior to the onset of zygotic

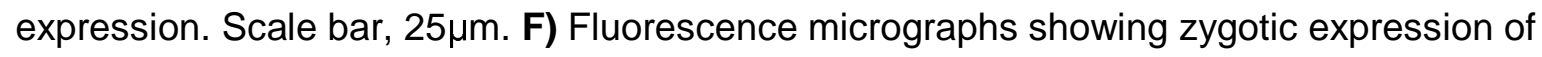
GFP::3xFLAG::CSR-1 in embryos, and L1 and L2 animals. Embryos were generated from crosses of wild-type hermaphrodites to gfp::3xflag:::csr-1 males. Scale bar, 10 $\mu \mathrm{m}$. G) Fluorescence micrographs showing a time lapse of nuclear localization of endogenously tagged CSR-1 (reflecting CSR-1b expression) in a developing 8-cell embryo over the course of six minutes. Scale bar, $25 \mu \mathrm{m}$.

\section{Figure 3. CSR-1a is expressed in the intestine.}

A) Fluorescence micrographs of animals expressing GFP::3xFLAG::CSR-1, GFP::3xFLAG::CSR-1b and GFP::3xFLAG::CSR-1a in the intestine. Scale bar, 50 $\mathrm{mm}$. B) Notable transcription factors enriched in binding within the promoters of each csr-1 isoform, from the ModERN project (68).

Figure 4. CSR-1b is the essential CSR-1 isoform, but CSR-1a is required for full fertility under stress.

A-D) Box plot of viable brood size counts for the strains expressing endogenously tagged total CSR-1 or csr-1a mutants compared to wild type $\mathrm{N} 2$, when reared at $20^{\circ} \mathrm{C}$ and $25^{\circ} \mathrm{C}$. Median is represented, with Tukey whiskers. Differences are not significant unless marked. Significance was determined with a one-way ANOVA (alpha=0.05) with Tukey's multiple comparison test. ** indicates significance of $p<0.01,{ }^{* * *}$ indicates significance of $p<0.001,{ }^{* * *}$ indicates significance of $p<0.0001$ A) Viable brood size for the strain endogenously tagged total CSR-1 vs. N2, when reared at $20^{\circ} \mathrm{C}$ and $25^{\circ} \mathrm{C}$. Number of individual $\mathrm{P}_{0}$ hermaphrodites counted for each genotype: $\mathrm{N} 220^{\circ} \mathrm{C}=14 ;$ gfp::3xflag::csr $-120^{\circ} \mathrm{C}=15$; $\mathrm{N} 225^{\circ} \mathrm{C}=14$; gfp::3xflag::csr $-125^{\circ} \mathrm{C}=13$. B) Viable brood size counts for the strains expressing endogenously tagged CSR-1b (red) and CSR-1a (blue) vs. N2, when reared at $20^{\circ} \mathrm{C}$ and $25^{\circ} \mathrm{C}$. Note that the endogenously tagged CSR-1b strain also carries a stop mutation in csr-1a. Number of individual $\mathrm{P}_{0}$ hermaphrodites counted for each genotype: $\mathrm{N} 220^{\circ} \mathrm{C}=16$; csr-1a(tor159), gfp::3xflag::csr-

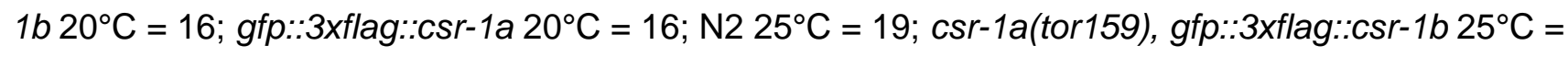
17; gfp::3xflag::csr-1a $\left.25^{\circ} \mathrm{C}=18 . \mathrm{C}\right)$ Viable brood size for csr-1a mutants vs. $\mathrm{N} 2$ reared at $20^{\circ} \mathrm{C}$ and 
$25^{\circ} \mathrm{C}$. Number of individual $\mathrm{P}_{0}$ hermaphrodites counted for each genotype: $\mathrm{N} 220^{\circ} \mathrm{C}=30 ; \operatorname{csr}-1 a^{\left[G 120^{*}\right]}$ $20^{\circ} \mathrm{C}=16 ; \operatorname{csr}^{-1} a^{[G 91 R]}=15 ; \mathrm{N} 22^{-} \mathrm{C}=14 ; \operatorname{csr}-1 a^{\left[G 120^{*}\right]} 25^{\circ} \mathrm{C}=16 ; c s r-1 a^{[G 91 R]} 25^{-} \mathrm{C}=16$. D) Viable brood size of csr-1a(tor159), gfp::3xflag::csr-1b animals vs. N2 reared at $25^{-} \mathrm{C}$. 0x Outcrossed worms were reared for $>25$ generations at $20^{\circ} \mathrm{C}$ before shifting to $25^{-} \mathrm{C}$ for brood assessment. $3 \times$ Outcrossed worms were crossed to wild-type animals before shifting to $25^{-} \mathrm{C}$ for brood assessment. Number of individual $\mathrm{P}_{0}$ hermaphrodites counted for each genotype: $0 x$ outcrossed $\mathrm{N} 2=18$; 0x outcrossed csr1a(tor159); gfp::3xflag::csr-1b = 12; 3x outcrossed N2 = 17; csr-1a(tor159), gfp::3xflag::csr-1b = 17. E) Viable brood size for the csr-1(tm892) null mutant and N2 vs. the csr-1(tm892) null mutant rescued with $c s r-1 b$ and csr-1a transgenes expressed under control of the $r p l-28$ promoter and reared at $20^{\circ} \mathrm{C}$. Number of individual $\mathrm{P}_{0}$ hermaphrodites counted for each genotype: $\mathrm{N} 2=33$; csr-1(tm892) = 20; csr1(tm892); $c s r-1 b$ rescue = 13; csr-1(tm892); csr-1a rescue = 18. F) Fluorescence micrographs showing germline expression of GFP::CSR-1 in animals rescued with each csr-1 isoform-specific transgene. Scale bar, $25 \mu \mathrm{m}$.

\section{Figure 5. CSR-1b and CSR-1a associate with different pools of sRNAs.}

A) Anti-FLAG western blots of GFP IPs used in sequencing GFP::3xFLAG::CSR-1a and GFP::3xFLAG::CSR-1b associated sRNAs. IPs were performed on recently outcrossed populations at $20^{\circ} \mathrm{C}$. B) Bar graph of first nucleotide and size distribution of normalized sRNA reads from Input and IP samples sequenced from GFP::3xFLAG::CSR-1b and GFP::3xFLAG::CSR-1a. C) Scatter plots of sRNAs in Input and IP samples of CSR-1b (left) and CSR-1a (right) broken down by genomic features. Each dot represents a gene. Teal dots represent transcripts/genes which have a minimum of 5RPM in IP samples and are 2-fold or greater enriched relative to Input in both replicates. Sense reads are counted for miRNAs and piRNAs, and antisense reads are counted for protein coding genes, transposons, and pseudogenes. The number of features enriched in the IP is shown $(\mathrm{N}=\#)$. For protein coding, transposons, and pseudogenes, only 22G-RNAs (defined as 21-23nt, with no first nucleotide bias) were considered for enrichment. RPM = reads per million. D) Venn Diagram showing the overlap between the enriched 22G-RNAs antisense to protein coding genes from CSR-1b (red, 
5326 total genes) and CSR-1a (blue, 2572 genes) IPs. E) Heatmap depicting the overlap between 22GRNA protein coding targets of CSR-1b only, CSR-1a only, and shared targets with the indicated data sets $(4,14,15,23,71)$. Numbers indicate the number of overlapping genes. Bold numbers indicate significant enrichment (Fisher's Exact Test, $\mathrm{p}<0.05)$. Enrichment $=$ number of overlapping genes divided by the expected number of overlapping genes drawn from two independent groups. F) Metagene plot of the distribution of enriched CSR-1b or CSR-1ba 22G-RNAs antisense to protein coding genes along the gene body. $\mathrm{N}=$ Number of genes.

\section{Figure 6: CSR-1 isoforms target shared and distinct sets of genes.}

A) Heatmap depicting the overlap between 22G-RNA protein coding targets of CSR-1b only, CSR-1a only, and shared targets with mutually exclusive germline constitutive, oogenic, and spermatogenic enriched transcript sets (29). Numbers indicate the number of overlapping genes. Bold numbers indicate significant enrichment (Fisher's Exact Test, $\mathrm{p}<0.05)$. Enrichment $=$ number of overlapping genes divided by the expected number of overlapping genes drawn from two independent groups. B) Tissue enrichment analysis of the 268 CSR-1a targets that do not overlap with the sets of germline transcript in A). C) Venn diagram of core histone genes enriched in the IPs for CSR-1b (red) and CSR1a (blue), relative to all histone genes in C. elegans. D) Bar graph showing the distribution of types of core histone genes enriched in each CSR-1 isoform IP.

\section{Figure 7. Loss of csr-1a affects different sets of genes in different tissues.}

A-B) Venn diagrams showing the overlap between genes that are differentially expressed in csr1a(tor159), gfp::3xflag::csr-1b (simply referred to here as csr-1a(tor159)) vs. wild-type worms at $20^{\circ} \mathrm{C}$ (A) (up-regulated genes: orange, down-regulated genes: purple) and $25^{\circ} \mathrm{C}(\mathrm{B})$ with the $22 \mathrm{G}-\mathrm{RNA}$ targets defined by CSR-1a and CSR-1b IPs. C) Tissue enrichment analysis (top) and Gene Ontology analysis (bottom) for genes that are down-regulated in csr-1a(tor159) vs. wild-type worms at $25^{\circ} \mathrm{C}$. D) Analysis of RPM in csr-1a(tor159) (blue) vs. wild-type worms (pink) at $25^{\circ} \mathrm{C}$ for genes in the germline constitutive, oogenic, and spermatogenic groups (29). E) Tissue enrichment analysis (top) and Gene 
Ontology analysis for genes that are over-expressed in csr-1a(tor159) vs. wild-type worms at $25^{\circ} \mathrm{C} \mathrm{F}$ ) Survival of worms at 72 hours treatment with Pseudomonas aeruginosa (PA14) in slow killing assays. Each data point represents one experiment, starting from at least 50 worms. Comparisons between samples were made using two-tailed $t$-tests.

\section{Figure 8. CSR-1 isoforms intersect differently with the WAGO-4 22G-RNA pathway.}

A) Venn diagram showing the overlap between CSR-1a/b 22G-RNA targets and WAGO-4 22G-RNA targets from L4 AGO IP samples. B) Heatmap depicting the overlap between 22G-RNA protein coding targets of CSR-1b only, CSR-1a only, WAGO-4 only, and various shared target groups with mutually exclusive germline constitutive, oogenic, and spermatogenic enriched transcript sets (29). Numbers indicate the number of overlapping genes. Bold numbers indicate significant enrichment (Fisher's Exact Test, $p<0.05)$. Enrichment $=$ number of overlapping genes divided by the expected number of overlapping genes drawn from two independent groups. C) Metagene plot of the distribution of WAGO4 enriched 22G-RNAs antisense to protein coding genes along the gene body. $\mathrm{N}=$ Number of genes. D) Pie charts of percentage of total reads with U-tailing (yellow-green; addition of one or more nontemplated uridines) for Input (top) and IP (bottom) samples of CSR-1b, CSR-1a, and WAGO-4 sRNAs (all genome matching sRNAs are included) E) Quantification of the number of GFP::3xFLAG::CSR-1a, GFP::3xFLAG::CSR-1b, and WAGO-4::GFP pixels that overlap with PGL-1::mRFP (burgundy) or HA::TagRFP::ZNFX-1 (blue) pixels in wild-type or mutant worms, using Mander's Correlation. For each data set, five $Z$ stacks of proximal germline regions from six different animals per strain were counted ( $N=30$ slices, approximately 80-100 nuclei per worm). Significance was analyzed as a mixed-effects ANOVA. ${ }^{* *}$ indicates significance of $p<0.01,{ }^{* * *}$ indicates significance of $p<0.001$.

\section{Figure 9. Four spermatogenesis AGOs contribute to sperm-mediated fertility.}

A) Fluorescence micrographs of L4 hermaphrodite germlines showing GFP::3xFLAG tagged CSR-1a, ALG-3, ALG-4, and WAGO-10 expression. Scale bar, 50 $\mu \mathrm{m}$. B) Box plot of median viable brood size for spermatogenesis ago mutants (with Tukey whiskers) at $25^{\circ} \mathrm{C}$. Single mutants were outcrossed 
before brood analysis and the quadruple ago mutant was outcrossed in the course of generating the strain. Differences are not significant unless marked. Significance was determined with a one-way ANOVA (alpha=0.05) with Tukey's multiple comparison test. ${ }^{* * * *}$ indicates significance of $p<0.0001$, and refers to all pairwise contrasts with the quadruple ago mutant. Number of individual $\mathrm{P}_{0}$ hermaphrodites counted for each genotype: N2 = 67; csr-1(tor159); gfp::3xflag::csr-1b = 12; alg$4(\operatorname{tm1184})=17 ; \operatorname{alg}-3(\operatorname{tm1155})=16 ;$ wago-10(tor31) = 17; alg-4; alg-3, csr-1a; wago-10 = 18 C) Box plot of median viable brood size for the quadruple spermatogenesis ago mutant vs. N2 (with Tukey whiskers) with or without mating to $\mathrm{N} 2$ males at $25^{\circ} \mathrm{C}$. Number of individual P0 hermaphrodites counted for each genotype: N2 = 10; N2 mated = 10; alg-4; alg-3, csr-1a; wago-10 = 10; alg-4; alg-3, csr-1a; wago-10 mated $=9$. Significance was determined with a one-way ANOVA (alpha=0.05) with Tukey's multiple comparison test. ${ }^{* *}$ indicates significance of $p<0.001$ and ${ }^{* * * *}$ indicates significance of $p<0.0001$. Representative images of progeny produced by each genotype are shown at the left (unfertilized oocytes or embryos). D) DAPI staining of whole L4 N2 or the quadruple spermatogenesis ago mutants. The spermatogenic gonad is outlined, and primary and secondary spermatocytes (defined by the appearance of the DNA and position), are marked. Scale bar is $25 \mu \mathrm{m}$. E) Quantification of PGL1::mRGFP (burgundy) or HA::TagRFP::ZNFX-1 (blue) in N2 and quadruple spermatogenesis ago mutant strains. For each data set, 20 nuclei from the germlines of five different animals were counted ( $N=100$ nuclei). Comparisons between strains for each type of granule are not significant, as evaluated as a mixed-effects ANOVA.

\section{Figure 10. Spermatogenesis AGOs target different subsets of germline genes.}

A) Clustering diagram of genes targeted by 22G-RNA binding AGOs and 26G-RNA binding AGOs during the L4 stage. Each blue line represents a single gene targeted by an AGO/sRNA. There are 7137 genes in total represented. B) Venn-pie diagrams comparing groups of genes targeted by each AGO (inner circle) with gene sets that define spermatogenesis-enriched transcripts, oogenesisenriched transcripts, and germline constitutive (outer circle) (29). The size of each color-coded circle represents the proportion of that sample that overlaps with the other sample. Numbers in the center of 
the circle indicate the number of genes that overlap between the two data sets. Numbers in bold are statistically significant as calculated by Fisher's Exact Test (one sided), $p<0.05$. C) Heatmap depicting the overlap between protein coding targets of various combinations of AGOs with mutually exclusive germline constitutive, oogenic, and spermatogenic enriched transcript sets (29). Numbers indicate the number of overlapping genes. Bold numbers indicate significant enrichment (Fisher's Exact Test, $p<0.05)$. Enrichment $=$ number of overlapping genes divided by the expected number of overlapping genes drawn from two independent groups. D-H) Clustering diagrams for various germline and spermatogenesis pathways depicting which AGOs target which genes in each pathway: D) Stem Cell Niche (80); E) Sex Determination (81); F) Spermatogenesis (22); G) Major Sperm Proteins (82); H) Sperm Activation (83).

Figure 11. Model for differential functions of CSR-1a and CSR-1b.

A model summarizing the expression patterns, sRNA partners, pathways, and functions of CSR-1 isoforms. 
Figure 1. CSR-1a and CSR-1b isoforms are differentially expressed throughout development

A

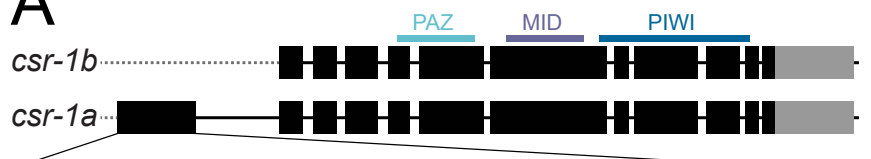

MQSDNVGRGRGSRGGSRGGSGRGRGRGGYGDSYRPDLETRETHVTYQGKVK

YEGGDRHDFNYEKTPTGSYRGSHRDGQEGSRGNRGSFRGGRGGYDRRRDDE

ESKAENRFGGYNRGANGGNYRGRTGGSGRGGSTQTTTVDPKYNGLILENSAEP

KLKGLNSD

Di-RG or Di-RGG Motifs

B

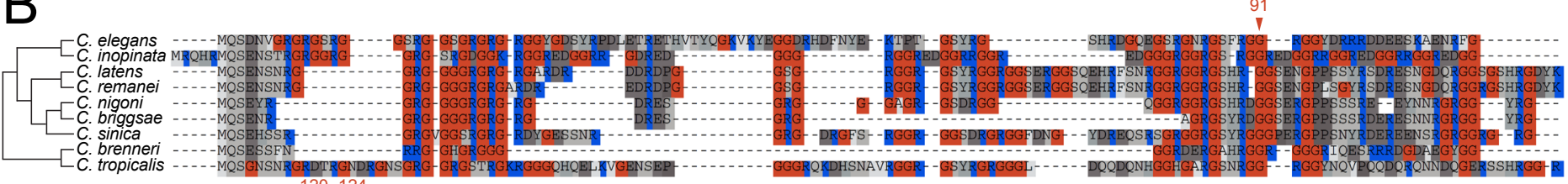
C. tropicalis 120124

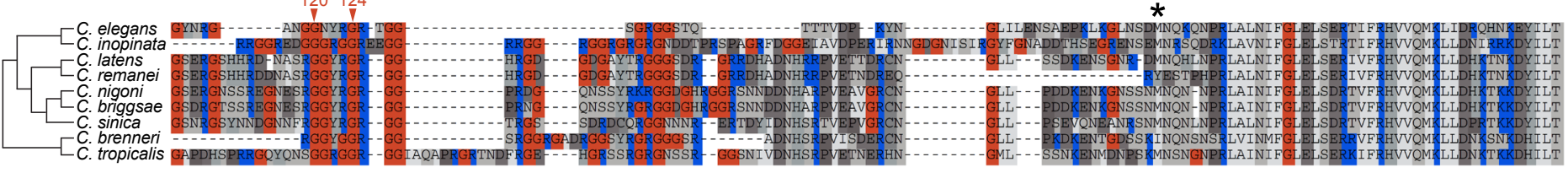

C

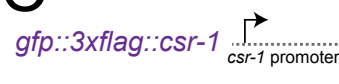

csr-1a(tor159);

gfp::3xflag:::csr-1b csr-1 promoter

gfp $:: 3 x f l a g::$ csr-1a $\underset{c s r-1 \text { promoter }}{\longrightarrow}$

cSr-1a $\mathrm{a}^{[\mathrm{G} 91 R]} \underset{\mathrm{CSr}-1 \text { promoter }}{\longrightarrow}$

$c s r-1 a^{\left[G 120^{*}\right]} \underset{c s r-1 \text { promoter }}{\longrightarrow}$
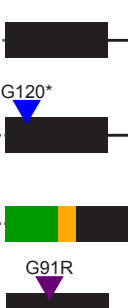

gfp 3xflag

matayta.

$\operatorname{csr-1}(\operatorname{tm} 892) \underset{\text { (tm892) }}{\longrightarrow}+\ldots$

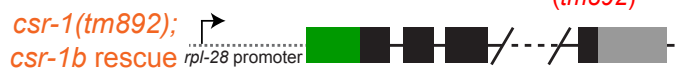

$c s r-1$ (tm892); $\quad$

csr-1a rescue rpp-28 promoter

HHOHA-

D
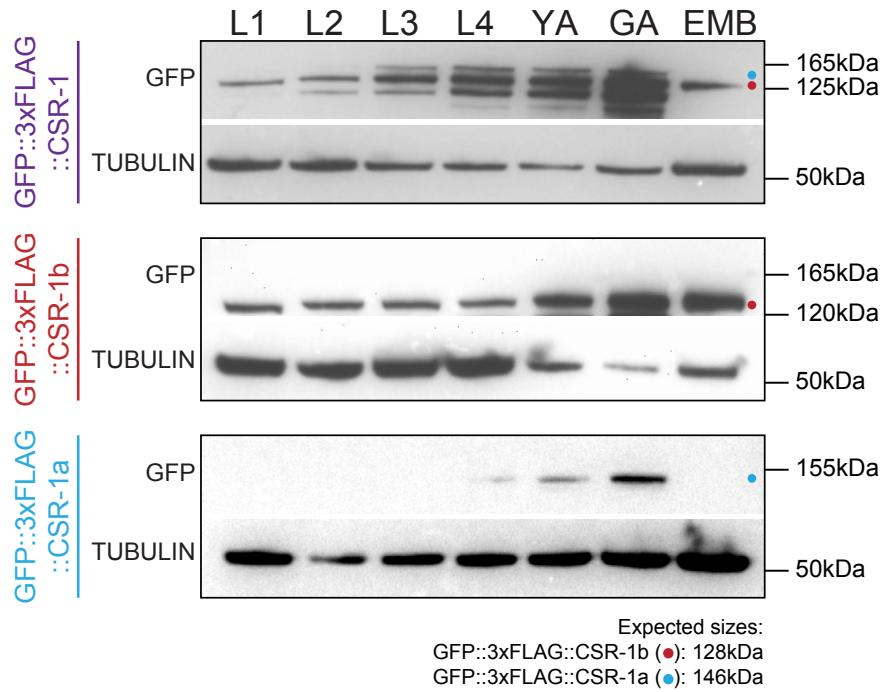

E
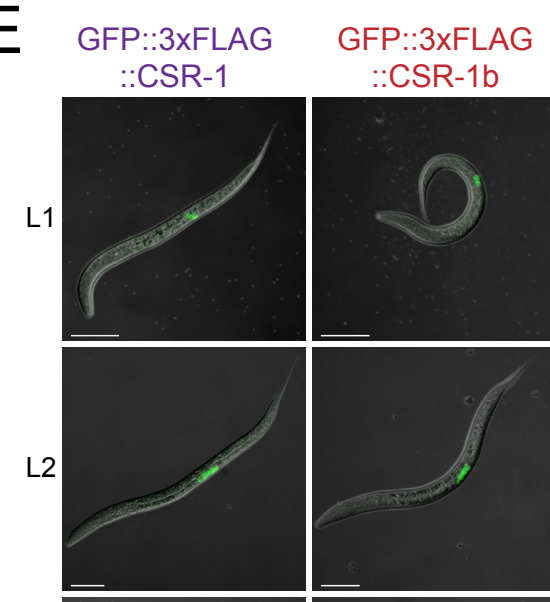

GFP::3xFLAG
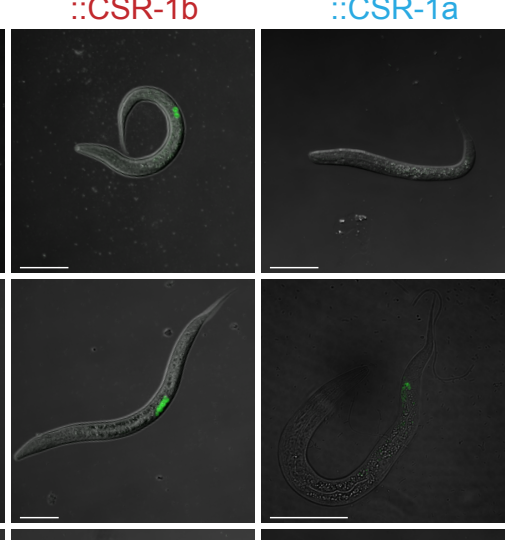

L3
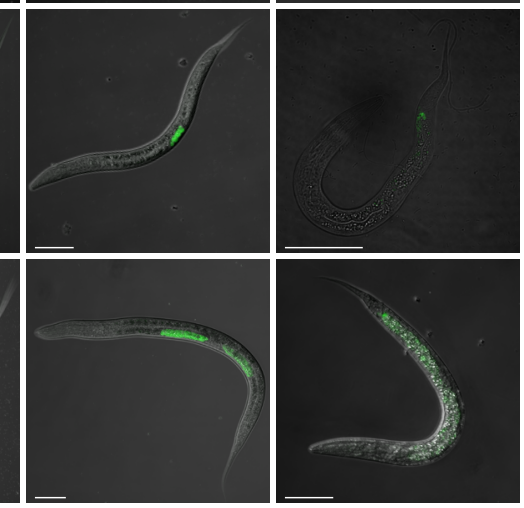
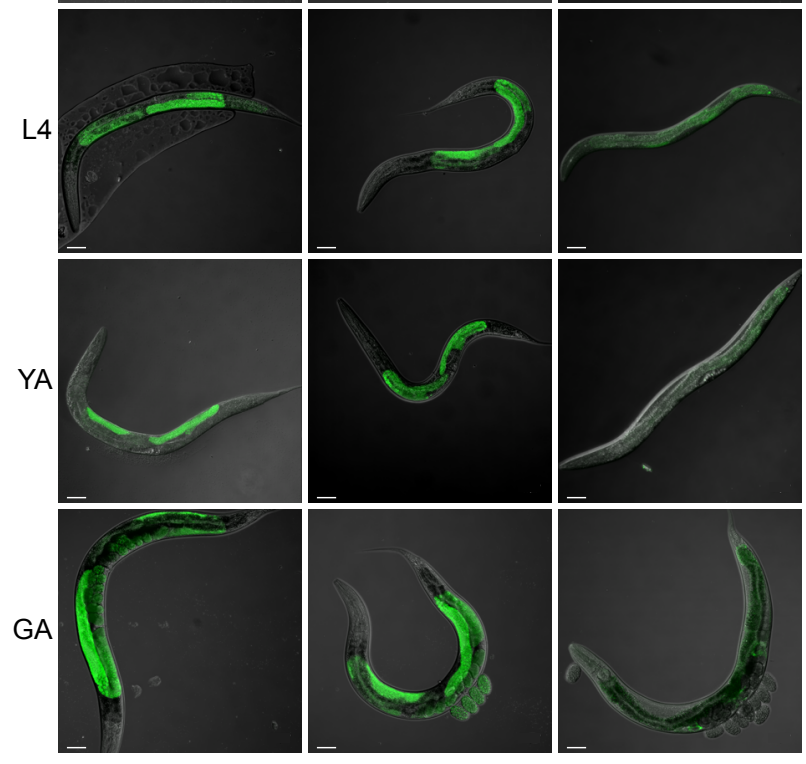
Figure 2. CSR-1a and CSR-1b isoforms are differentially expressed in the germline

A
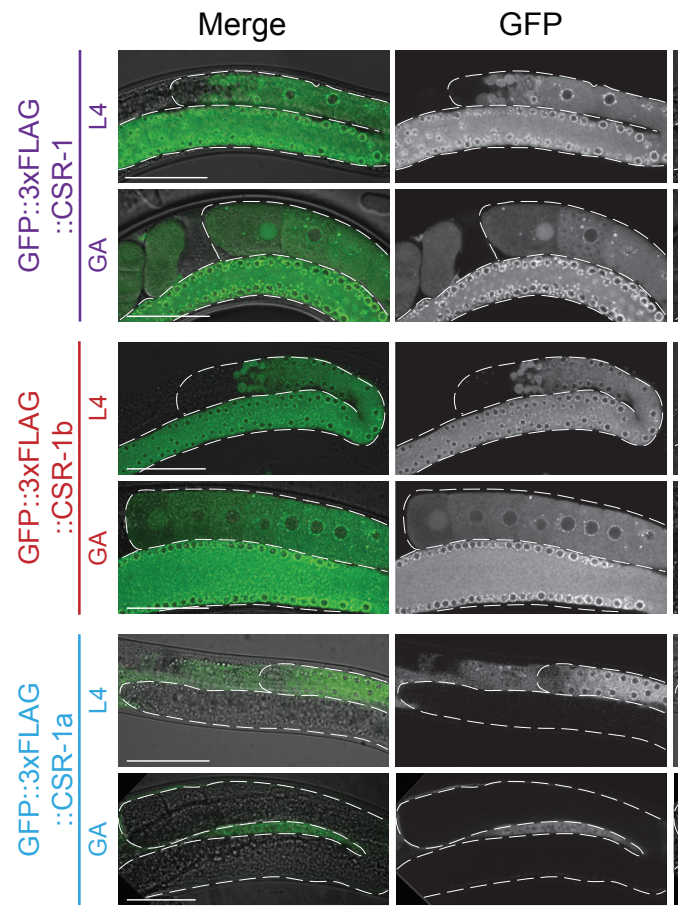

B
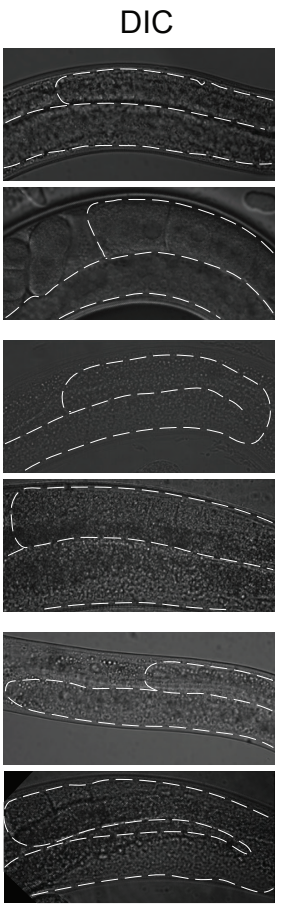

C

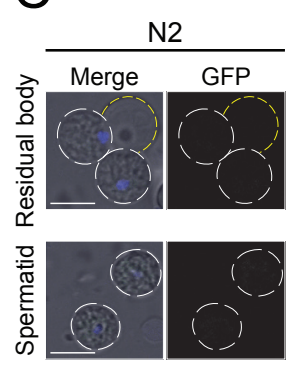

E
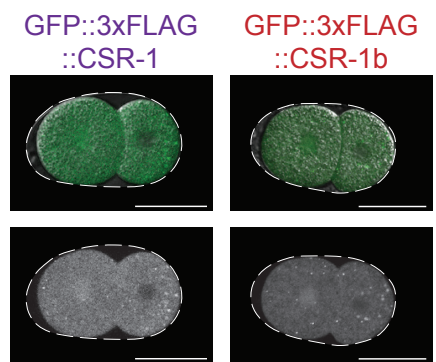

\section{G}

\section{GFP::3xFLAG::CSR-1}

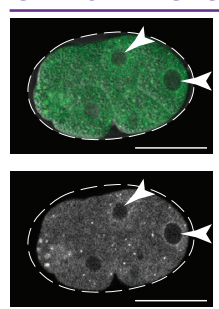

$\mathrm{t}=0 \min$
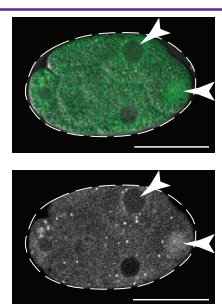

$\mathrm{t}=2 \min$

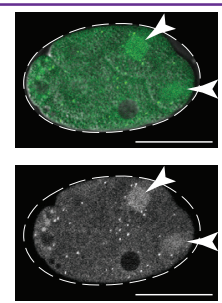

$\mathrm{t}=4 \min$
GFP $\cdot 3 \times$ FLAG
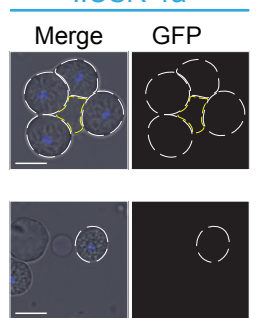

GFP ::3xFLAG
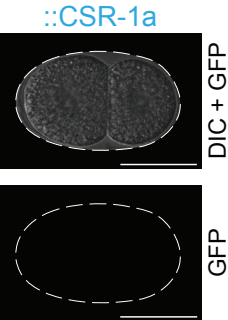

닌 $\mathrm{t}=6 \mathrm{~min}$

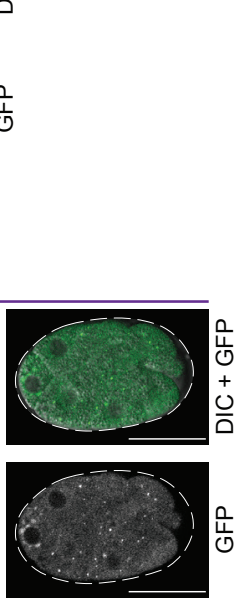

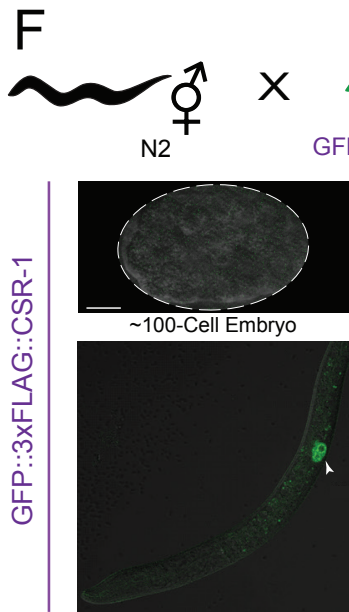

L1
PGL-1
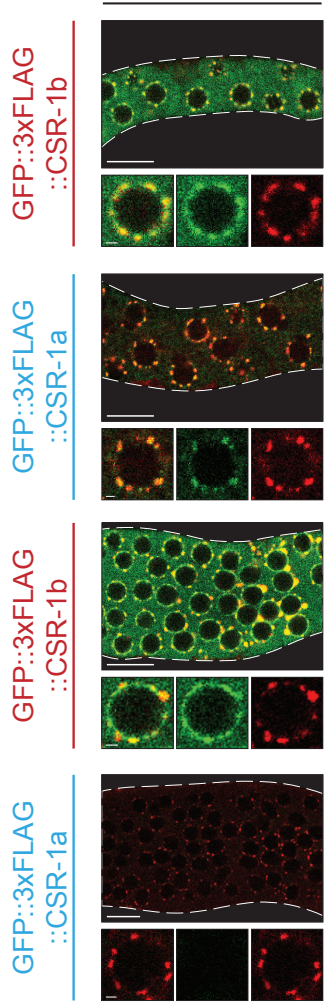

D

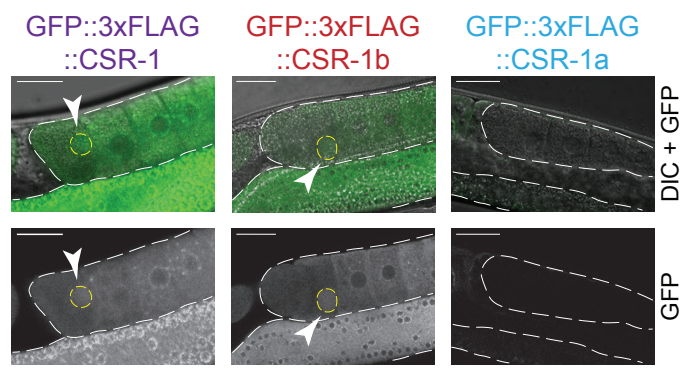

GFP::3xFLAG::CSR-1
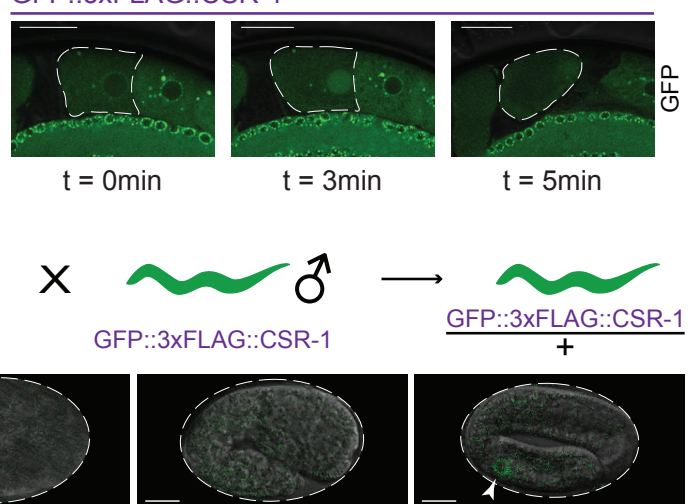

Comma Embryo

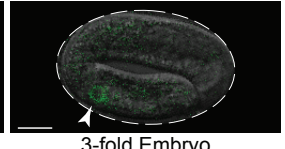

3-fold Embryo
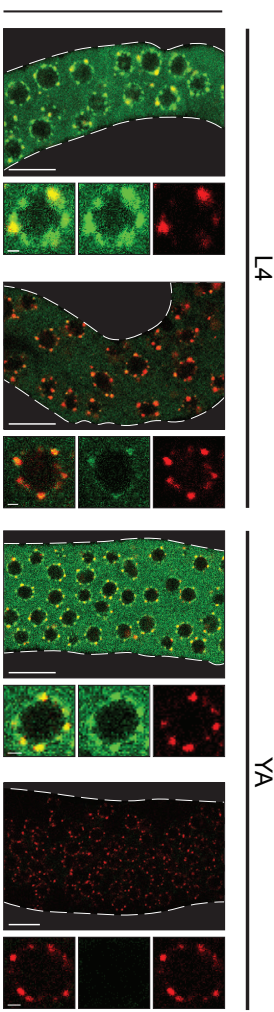

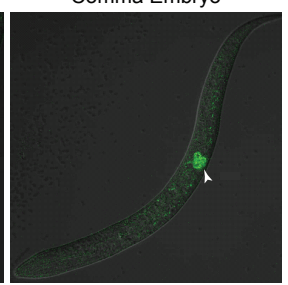

L2 
Figure 3. CSR-1a is expressed in the intestine

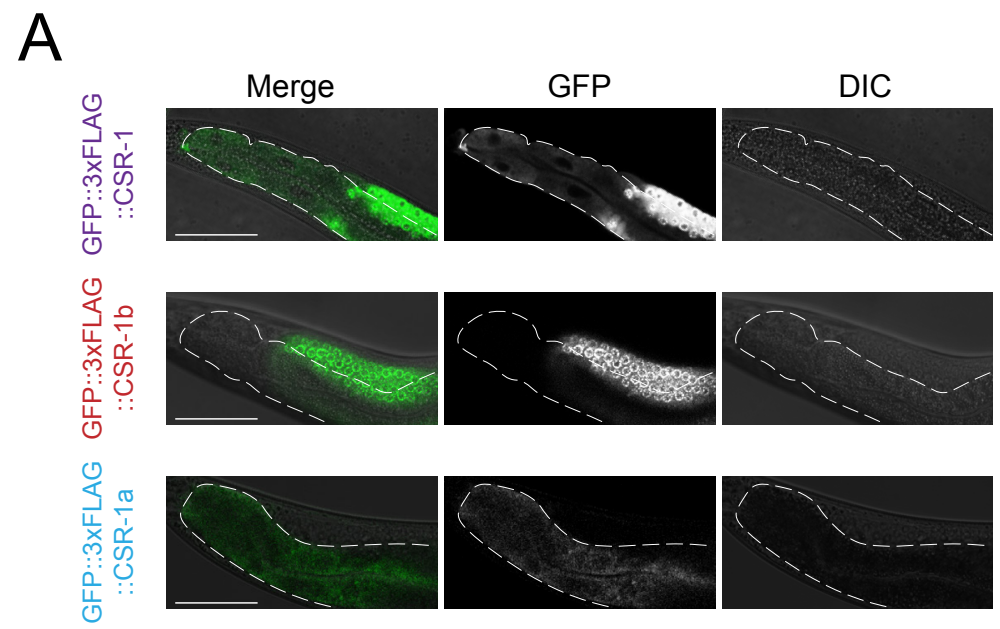

B

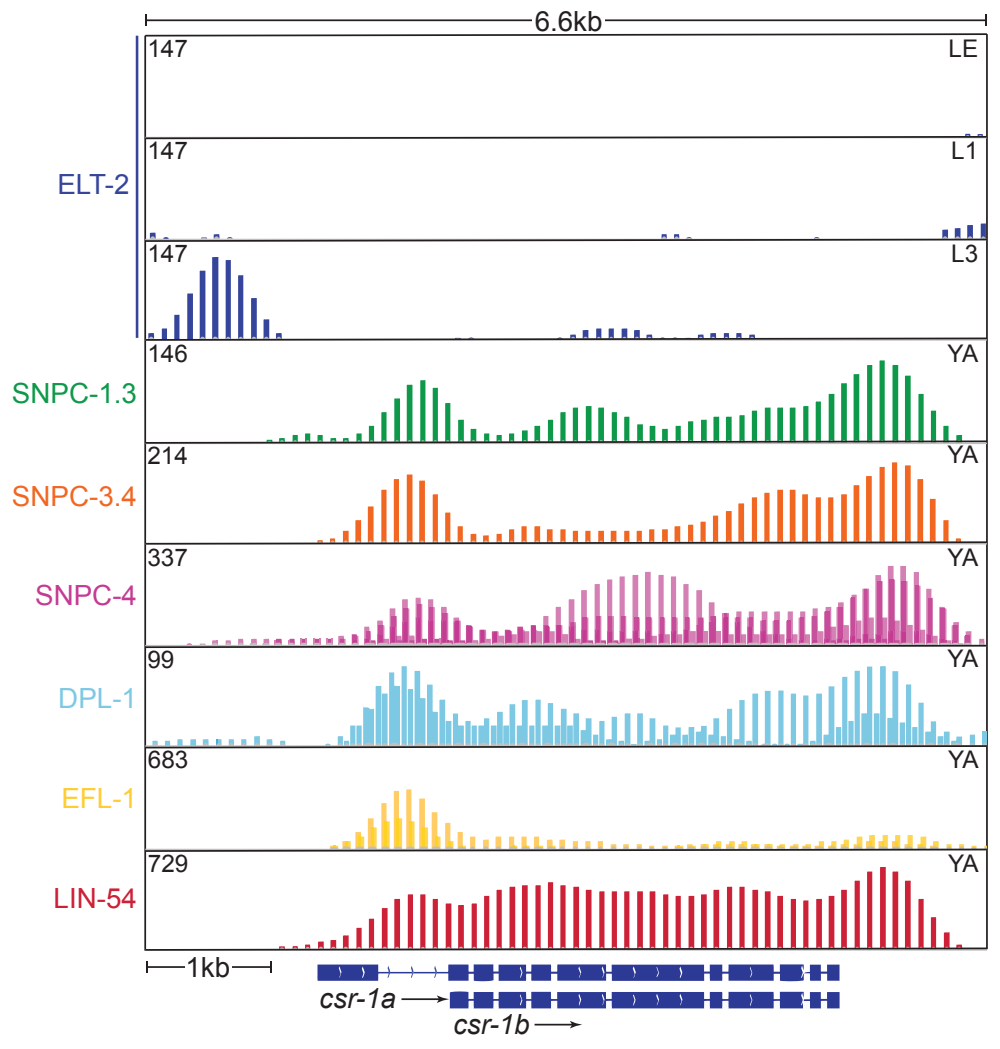


Figure 4. CSR-1b is the essential CSR-1 isoform, but CSR-1a is required for full fertility under stress
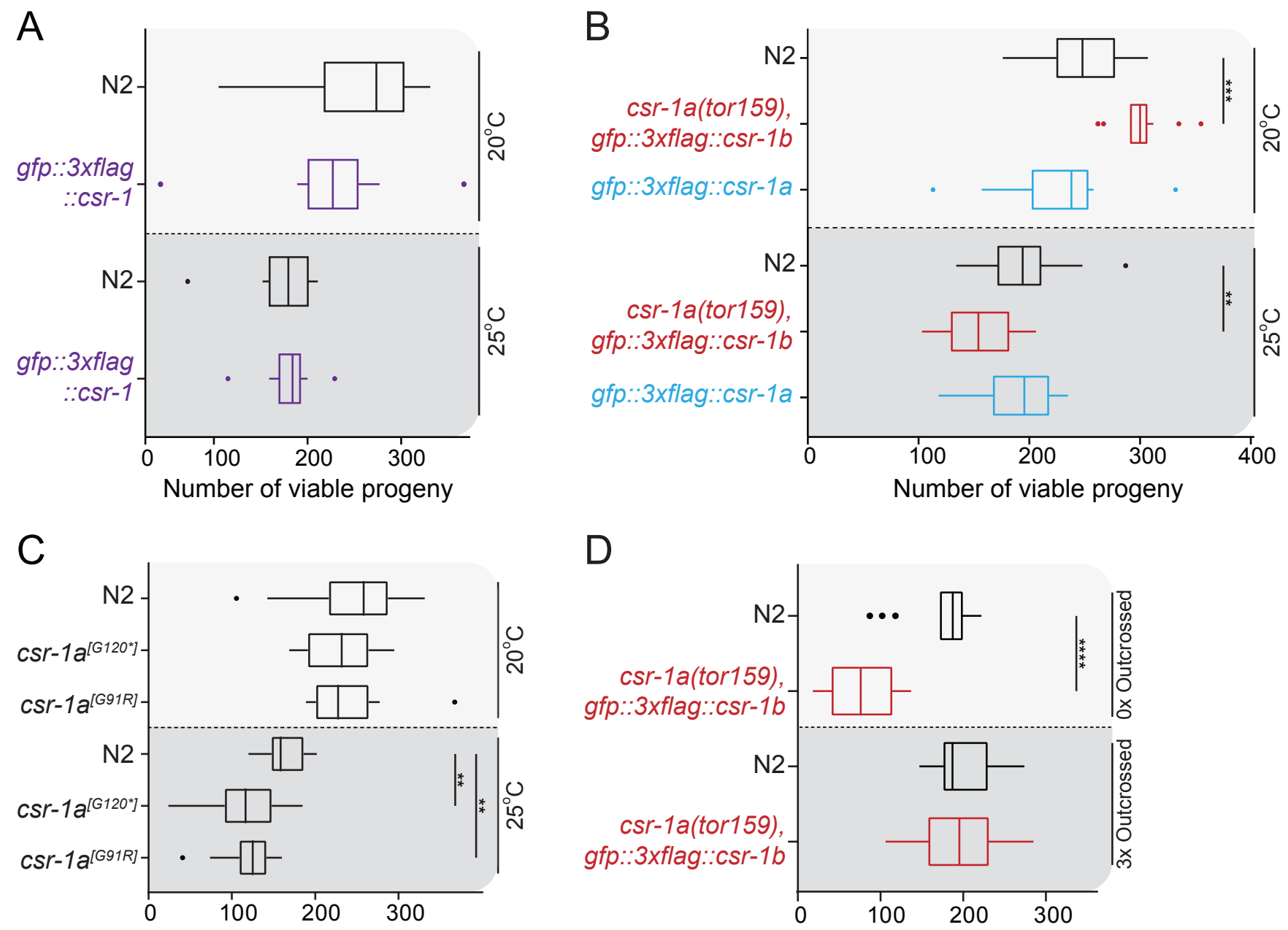

Number of viable progeny

Number of viable progeny
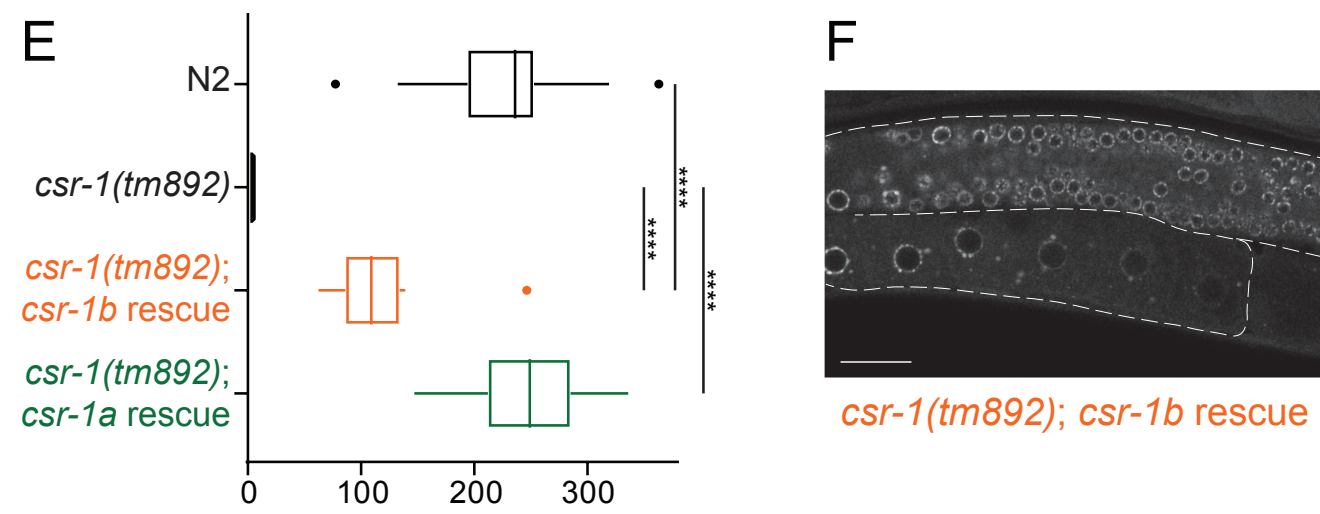

csr-1(tm892); csr-1b rescue

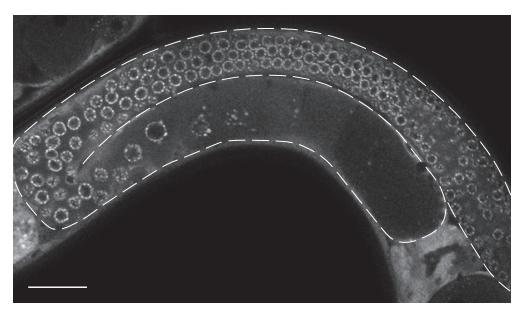

csr-1(tm892); csr-1a rescue

Number of viable progeny 
Figure 5. CSR-1 isoforms bind to distinct and overlapping pools of 22G-RNAs

A

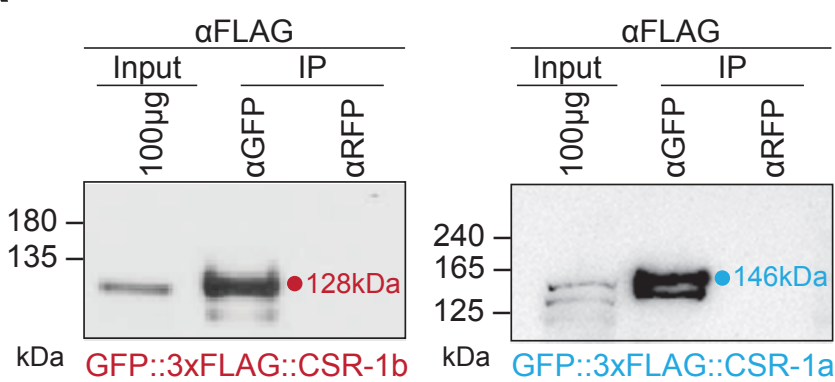

C
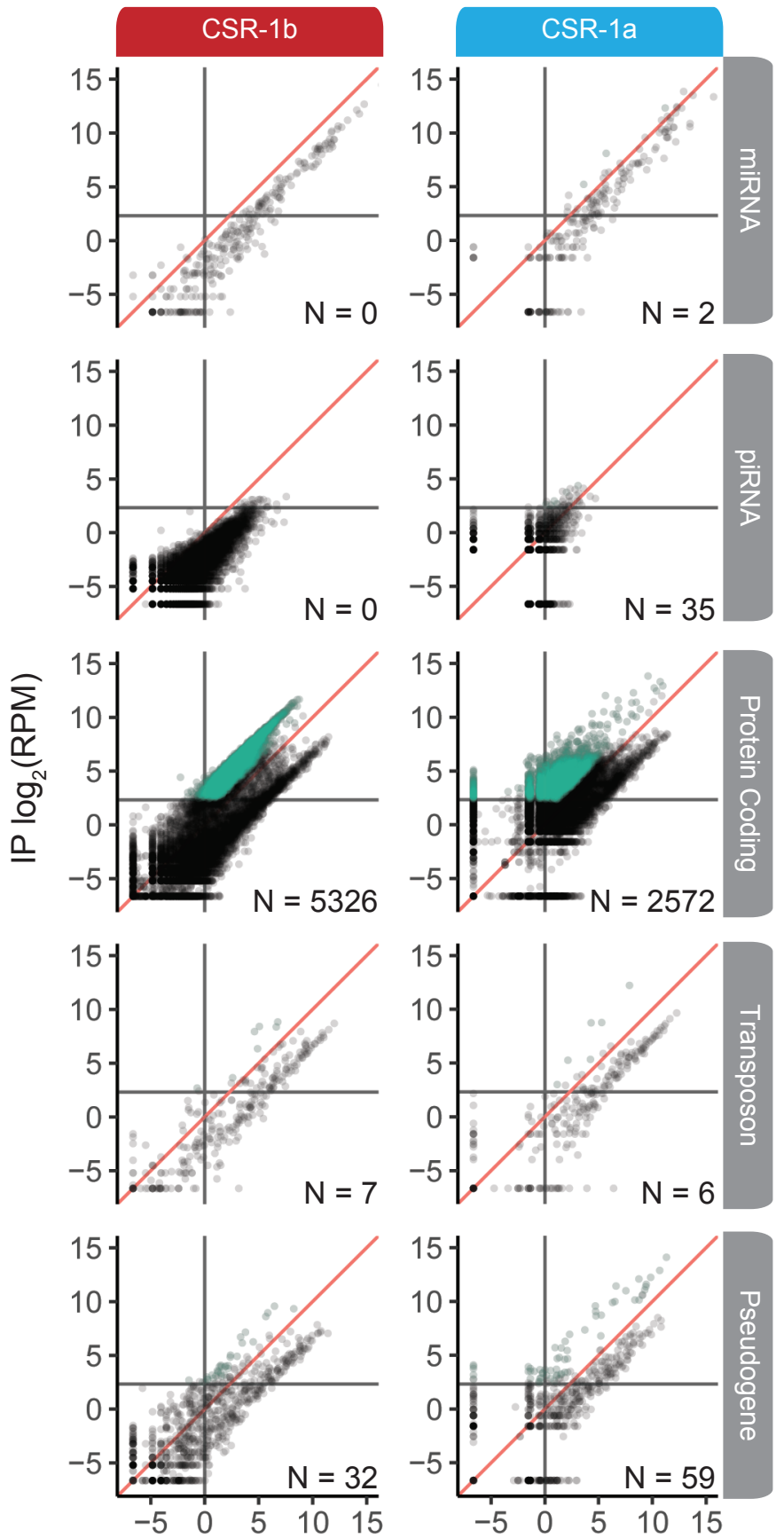

Input $\log _{2}(\mathrm{RPM})$
B
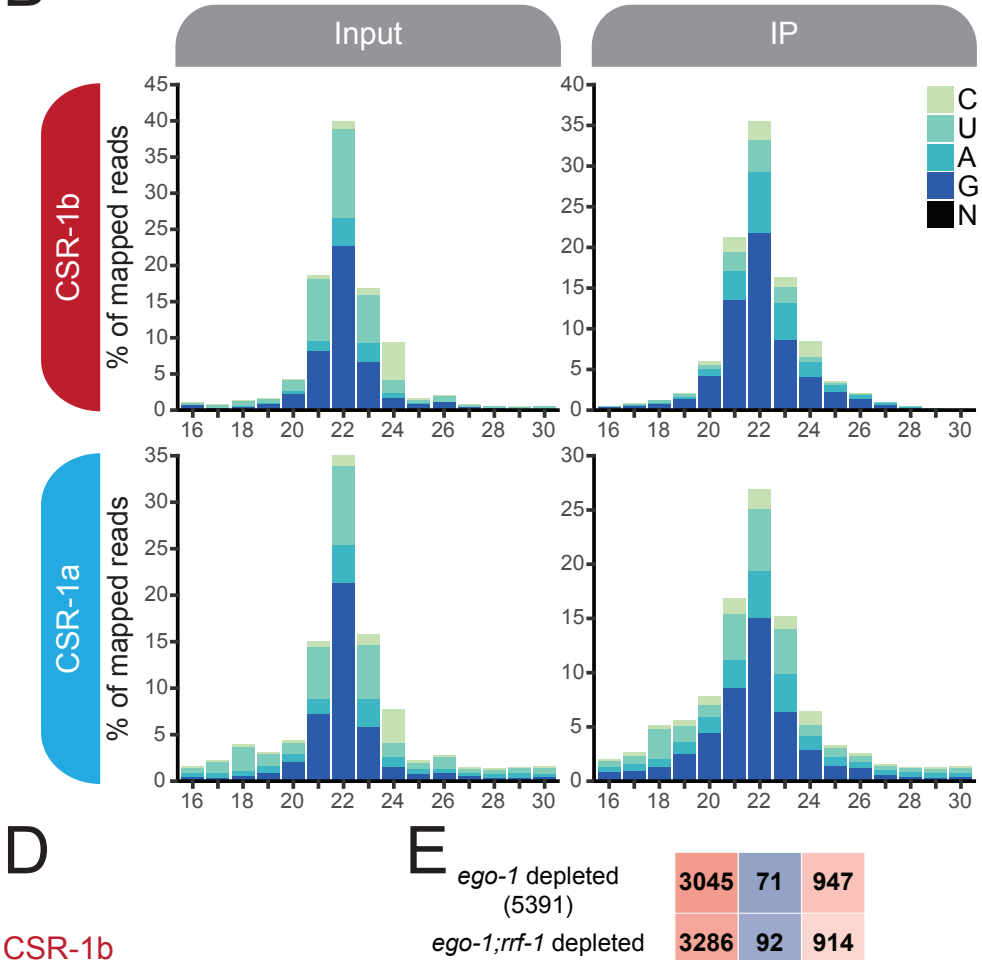

(5326)

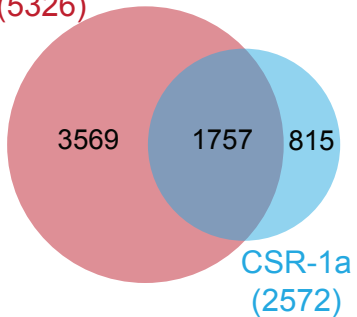

E ego-1 depleted (5391)

ego-1;rrf-1 depleted (6585)

rrf-1 depleted (130)

rrf-3 depleted (317)

alg-3;alg-4 depleted (1413)

WAGO-1 IP (1121)

CSR-1 male IP (5558)

CSR-1 hermaphrodite IP $2982 \quad 31 \quad 923$ (4185)

F
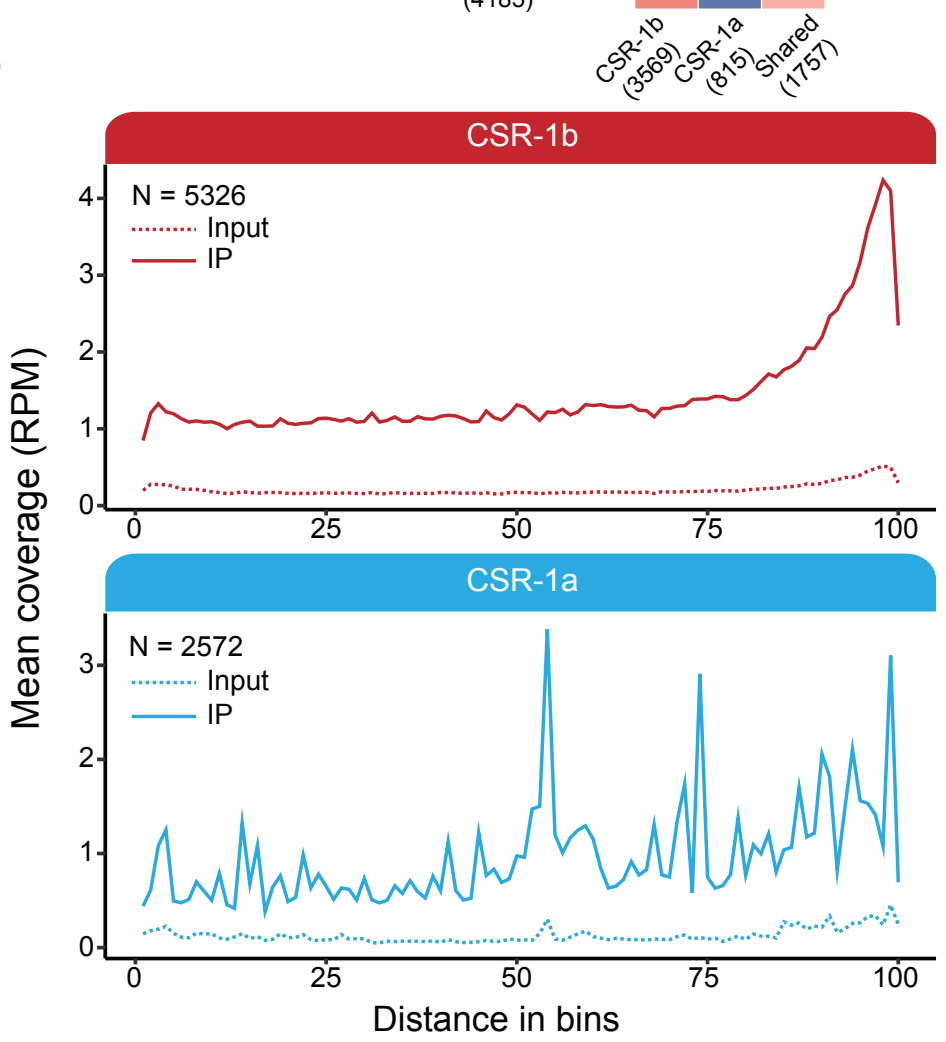
bioRxiv preprint doi: https://doi.org/10.1101/2020.07.20.212050; this version posted June 16, 2021. The copyright holder for this preprint (which was not certified by peer review) is the author/funder. All rights reserved. No reuse allowed without permission.

Figure 6. CSR-1 isoforms target shared and distinct sets of genes

A

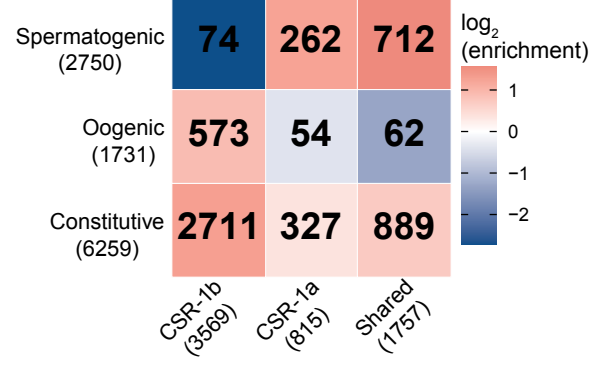

B

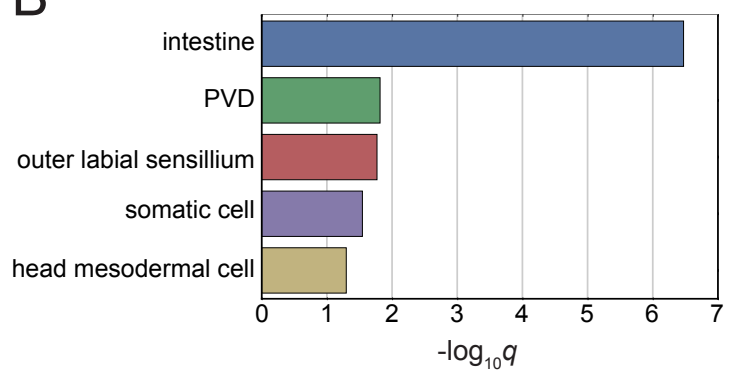

C

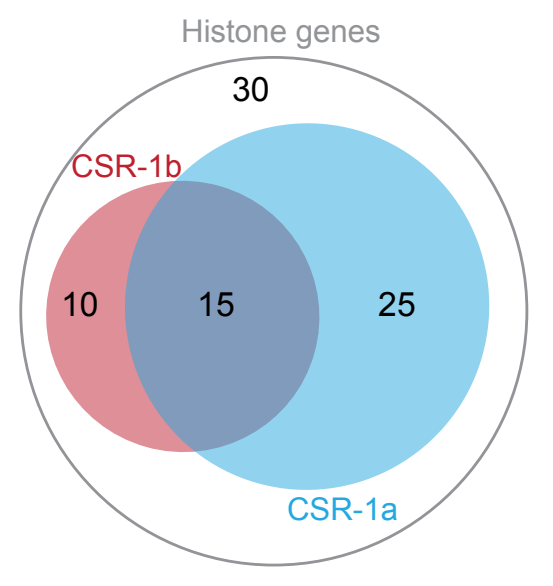

$\mathrm{D}$

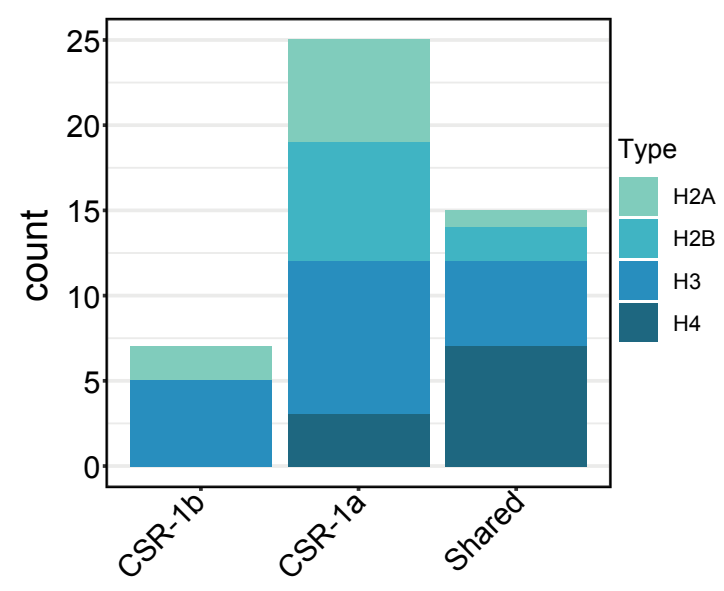


Figure 7. Loss of csr-1a affects different sets of genes in different tissues

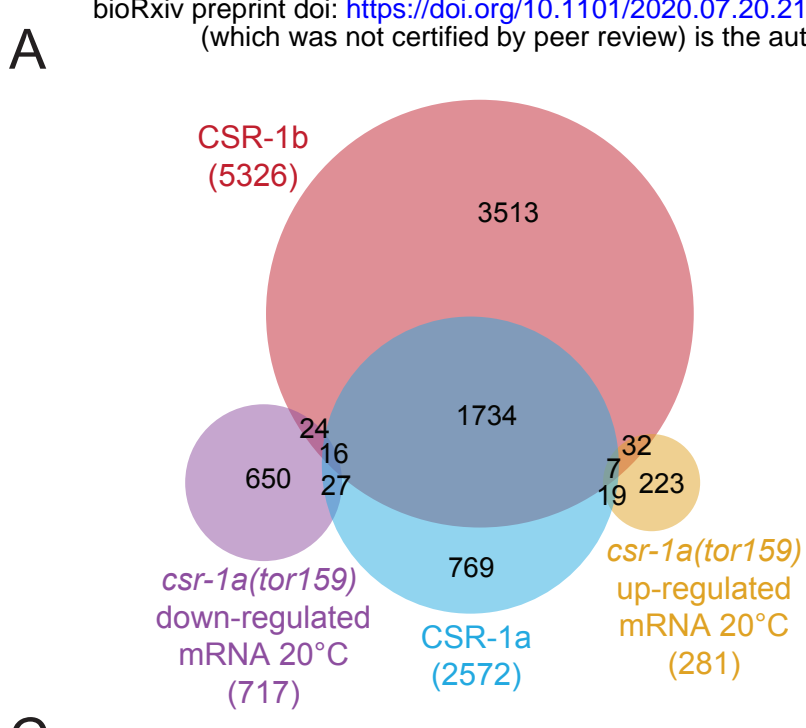

C

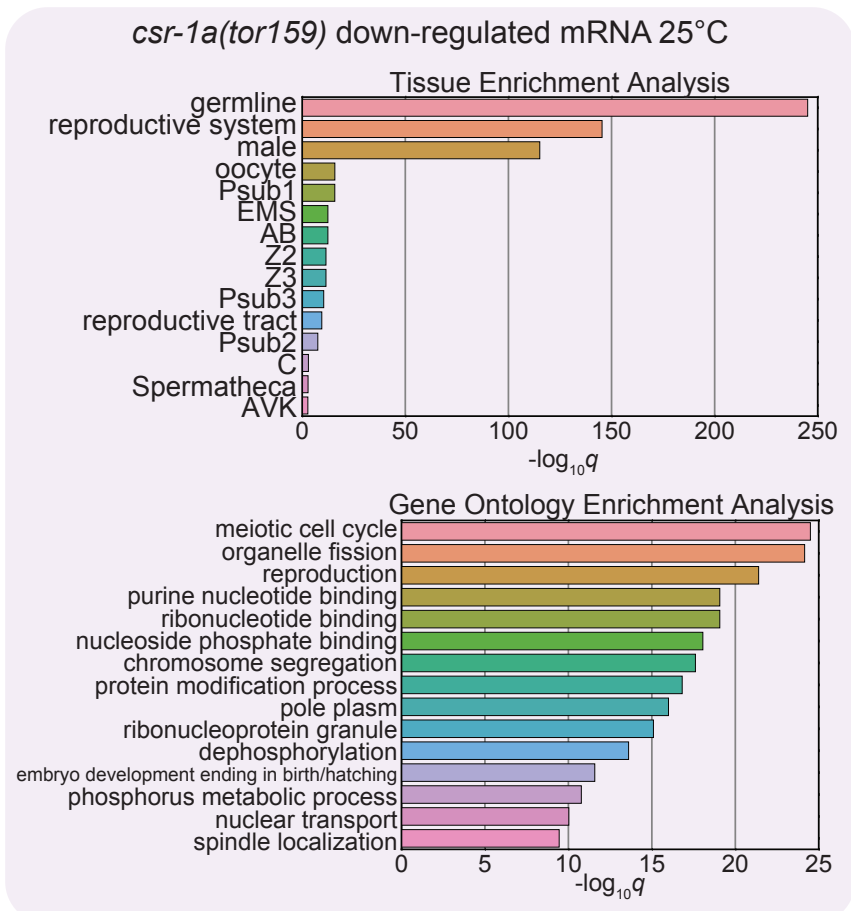

$\mathrm{D}$

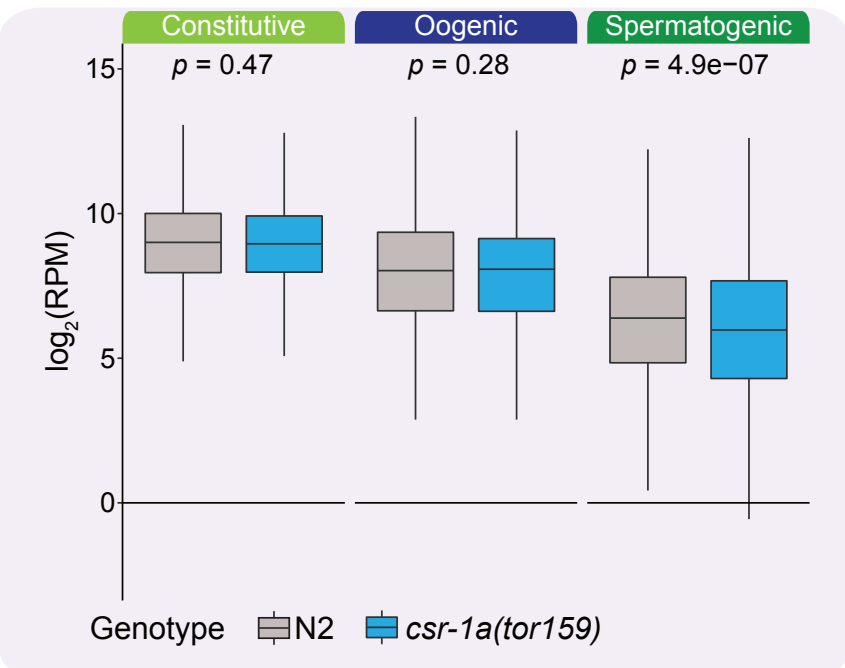

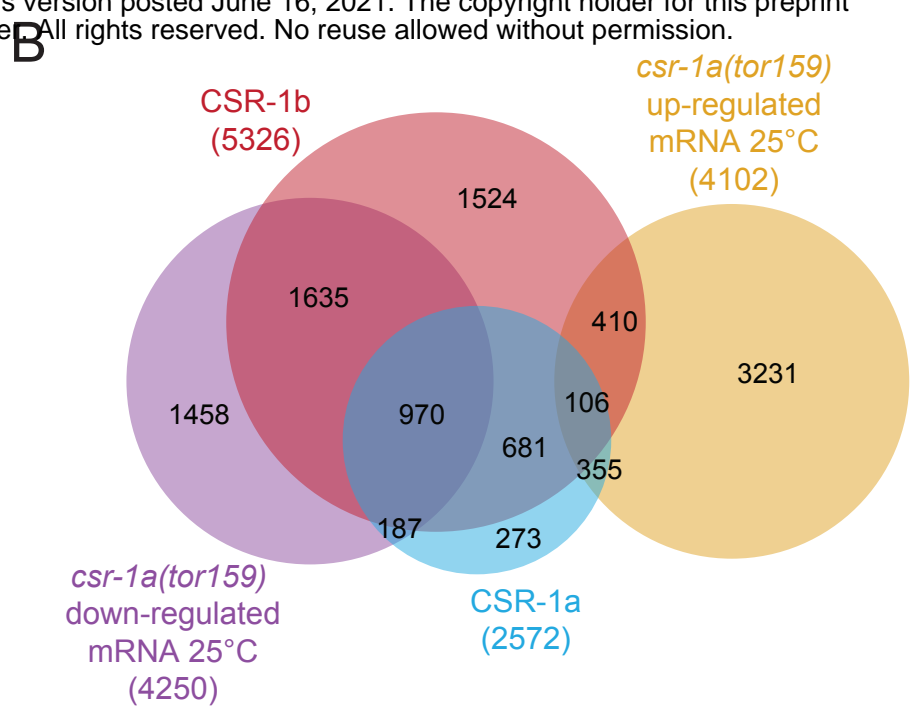

$E$

csr-1a(tor159) up-regulated mRNA $25^{\circ} \mathrm{C}$
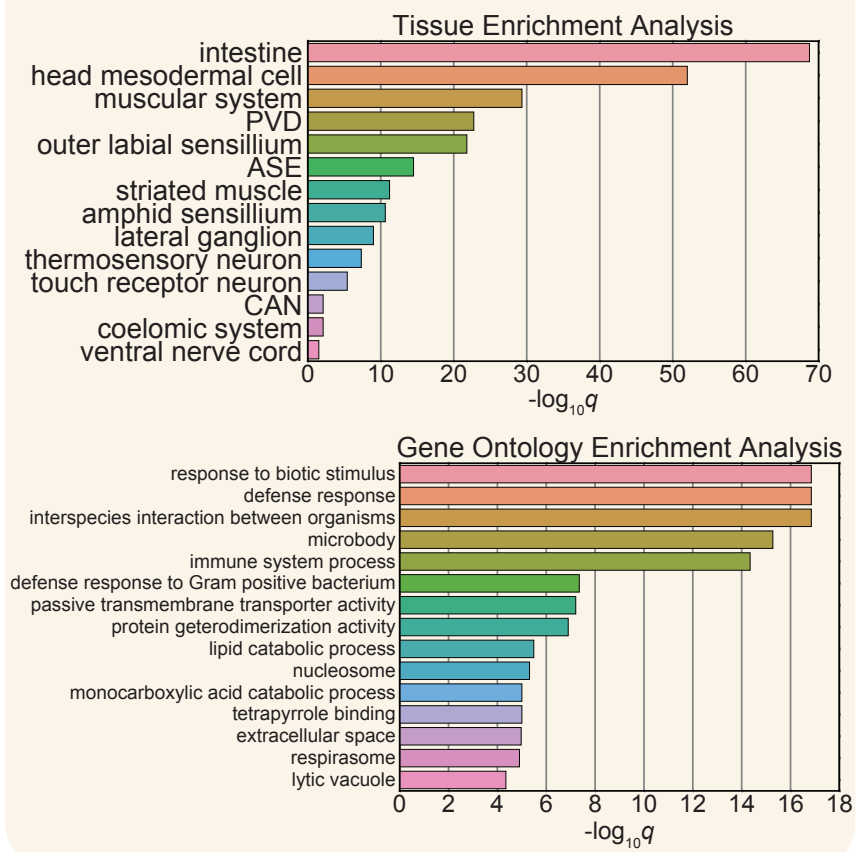

F

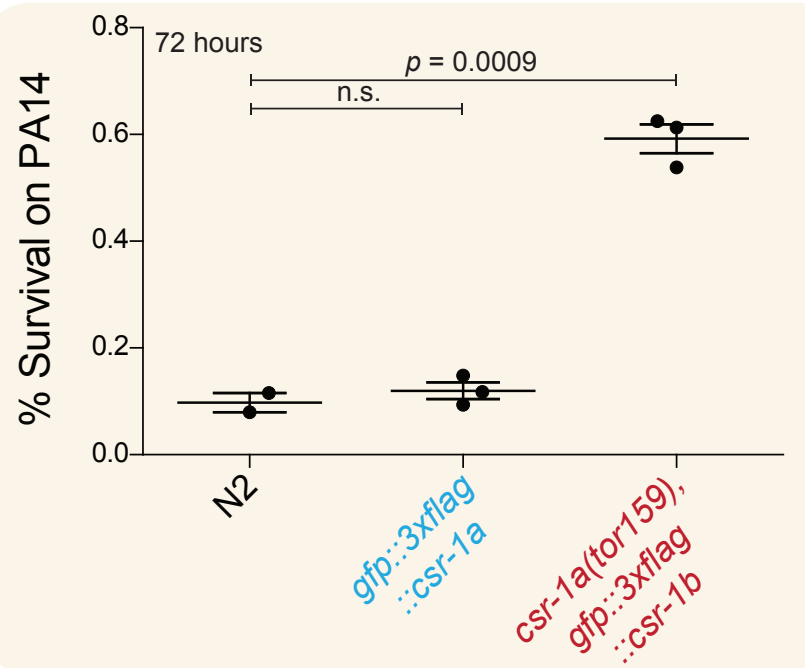


Figure 8. CSR-1 isoforms intersect differently with the WAGO-4 22G-RNA pathway

A

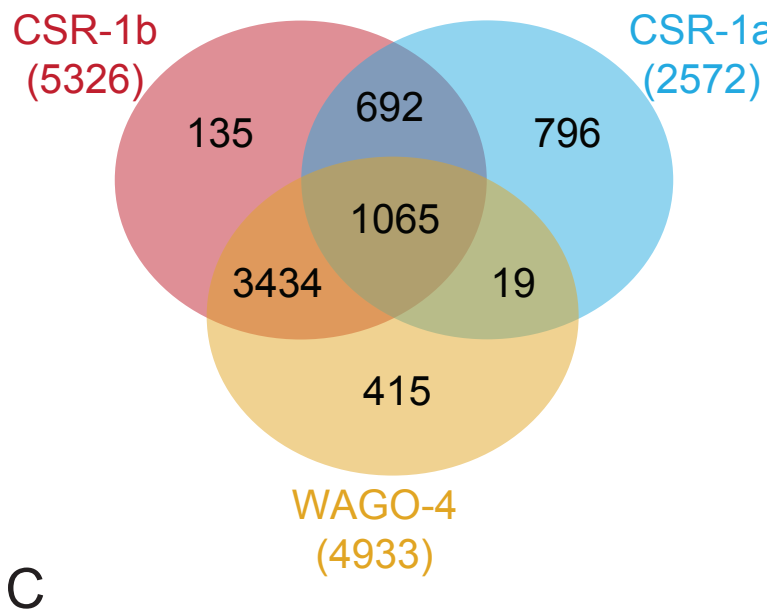

B

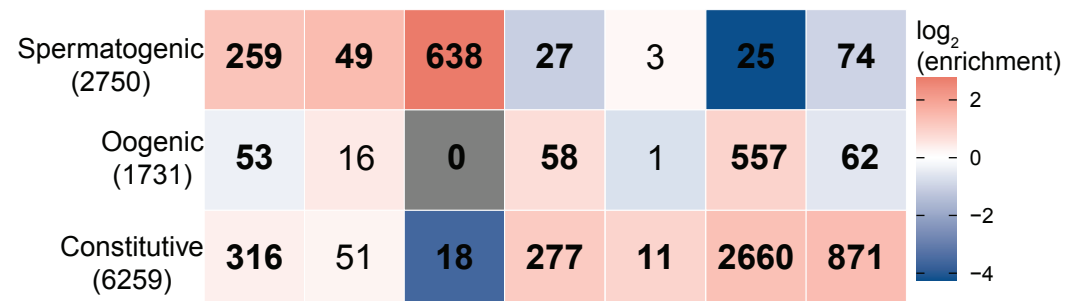

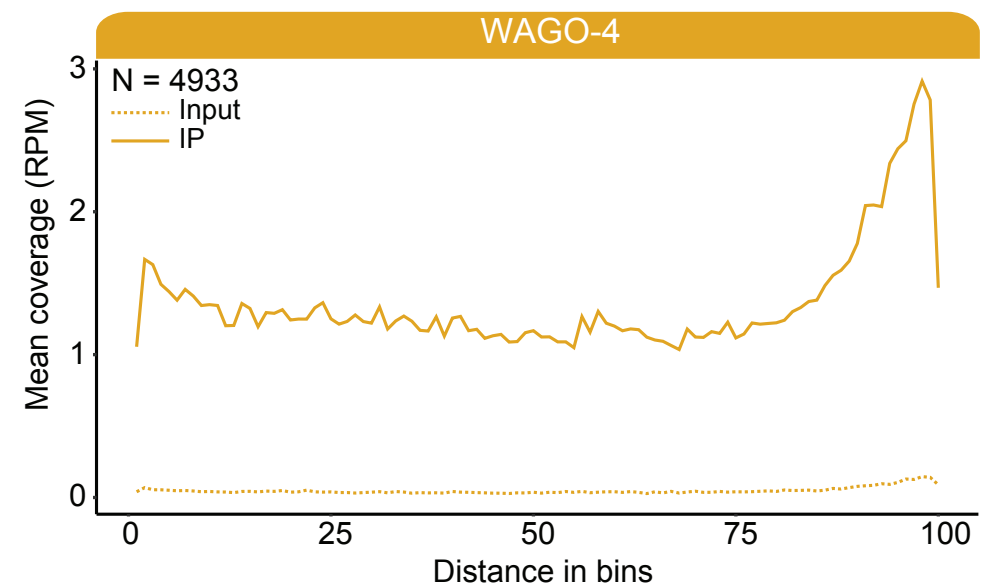

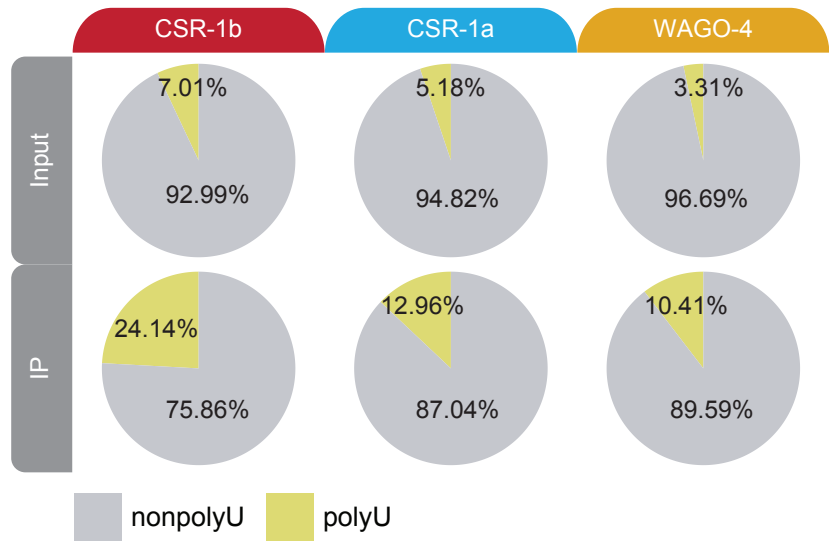

E

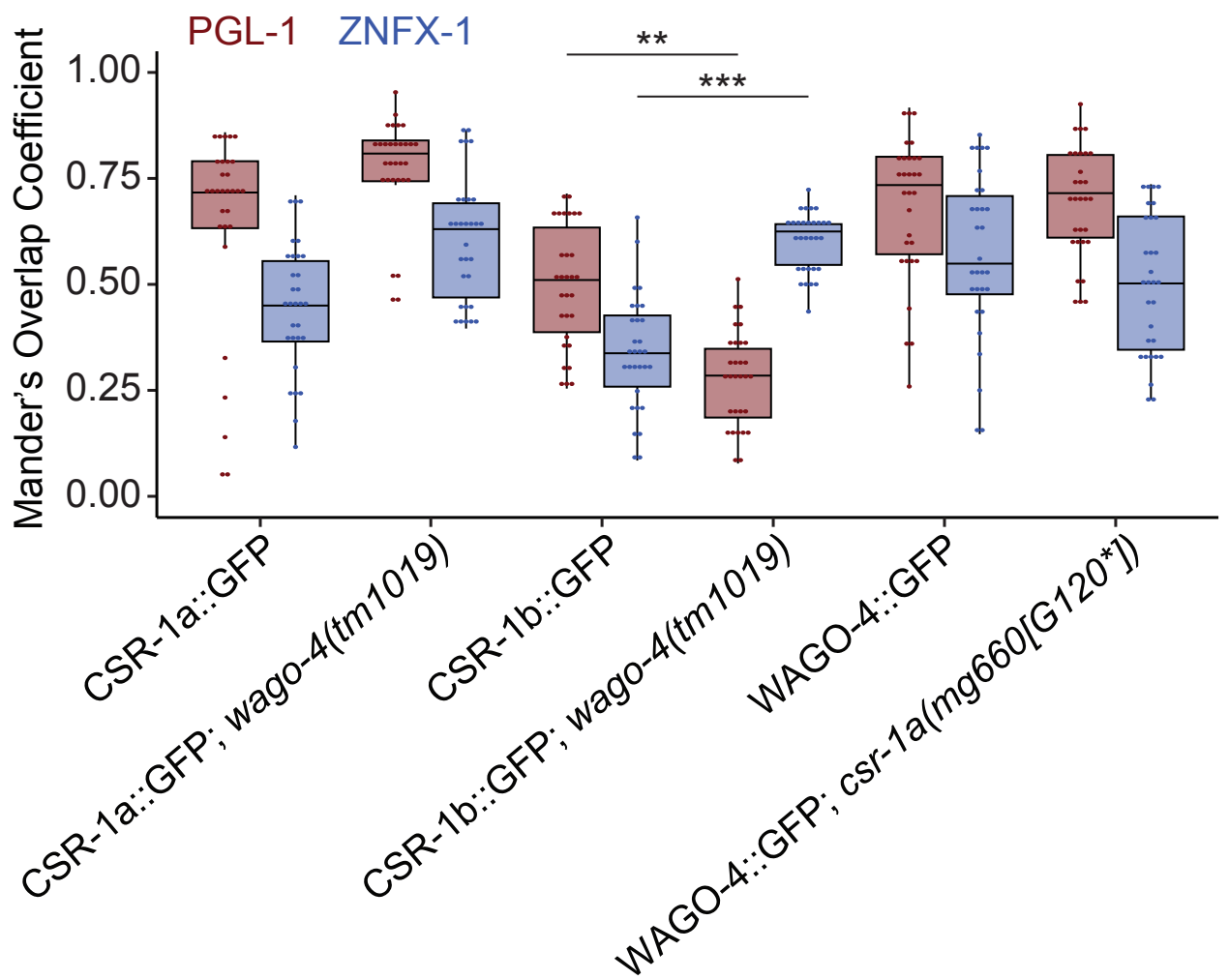


Figure 9. Four Spermatogenesis Argonautes contribute to sperm-mediated fertility

A
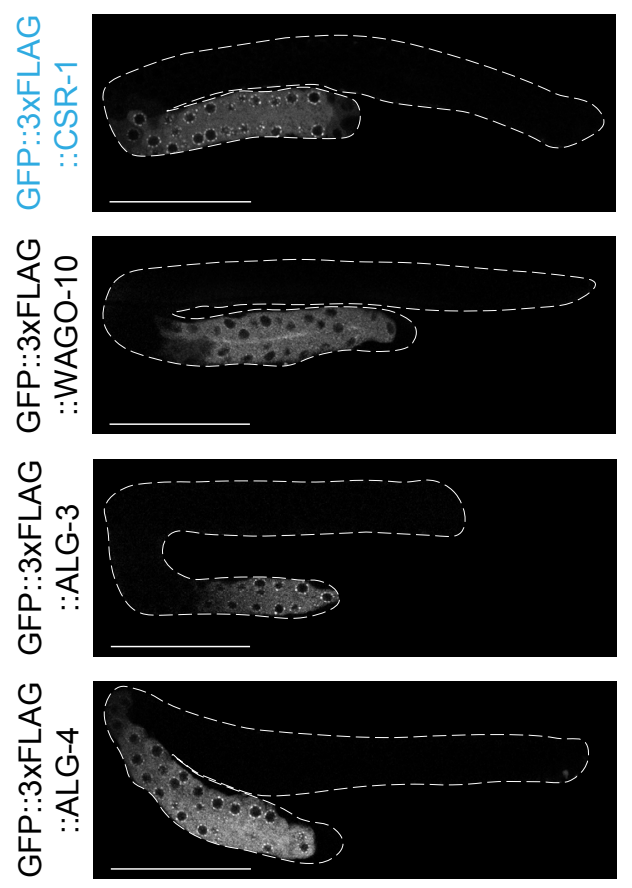

C

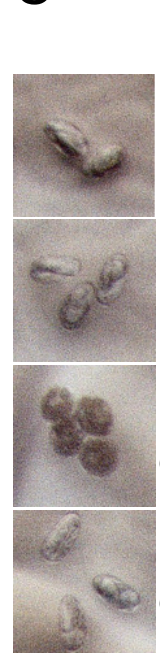

Hermaphrodite

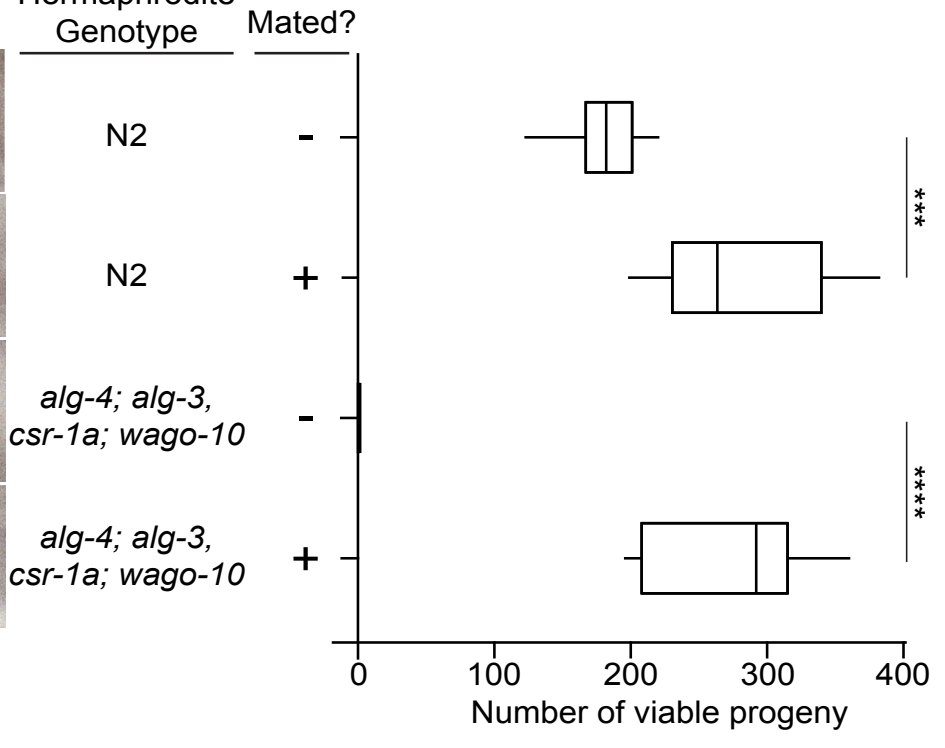

E

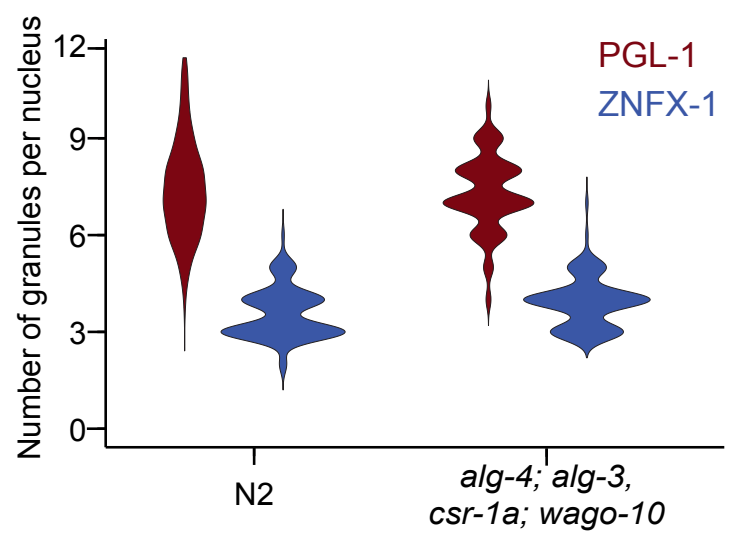

B

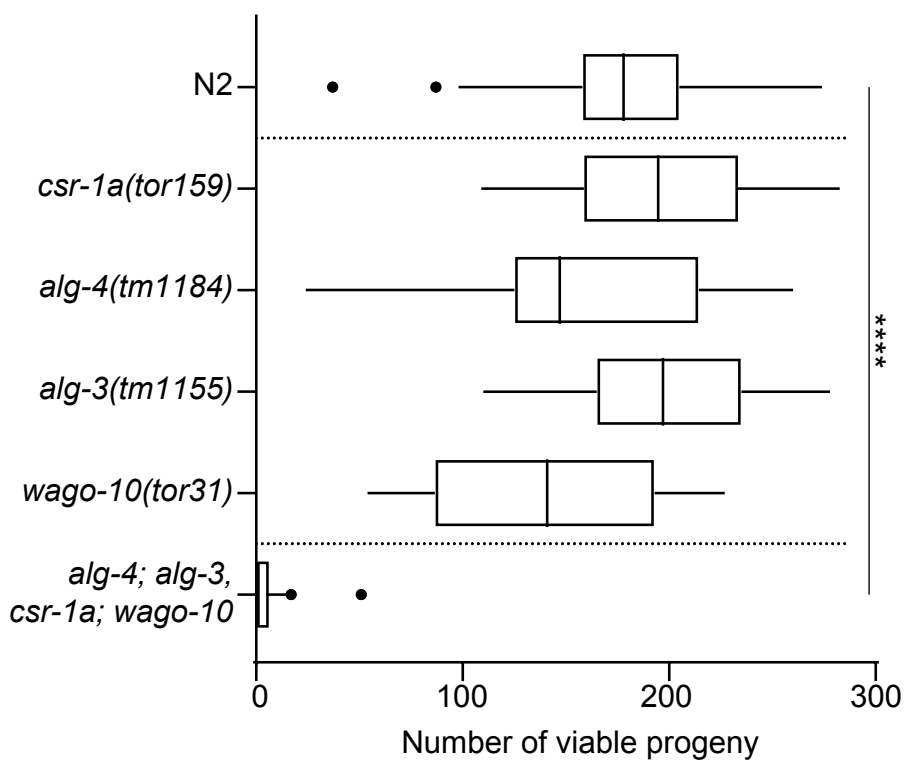

D
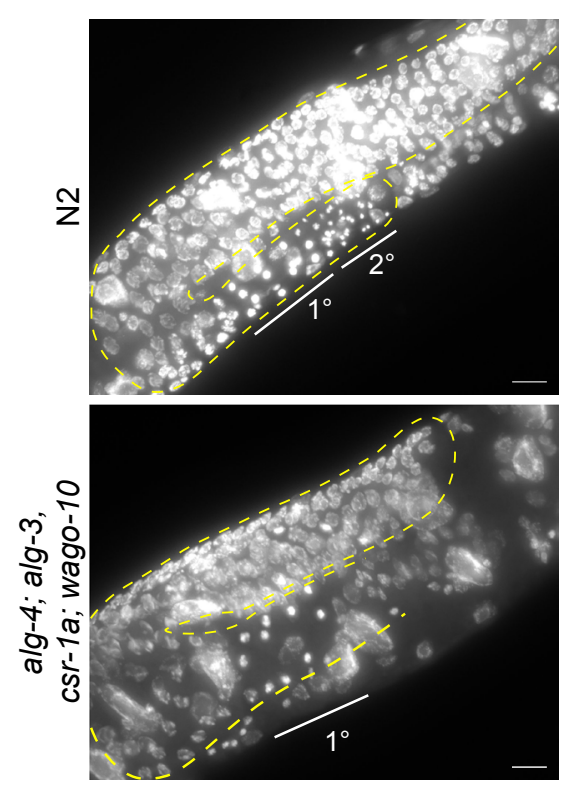
bioRxiv preprint doi: https://doi.org/10.1101/2020.07.20.212050; this version posted June 16, 2021. The copyright holder for this preprint (which was not certified by peer review) is the author/funder. All rights reserved. No reuse allowed without permission.

Figure 10. Spermatogenesis AGOs target different subsets of germline genes

A

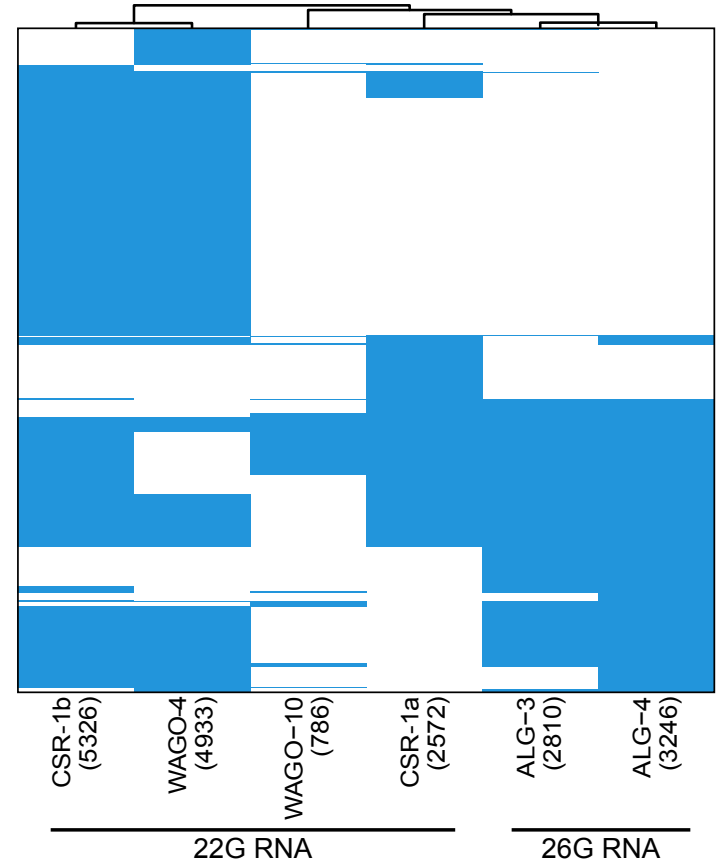

C

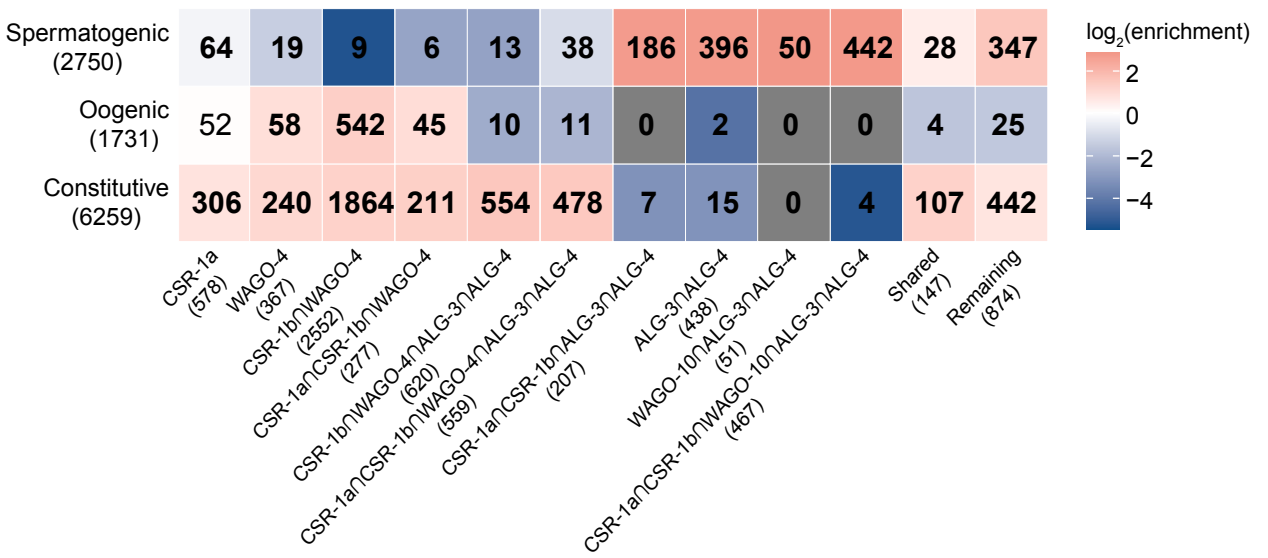

$\mathrm{E}$

Sex Determination
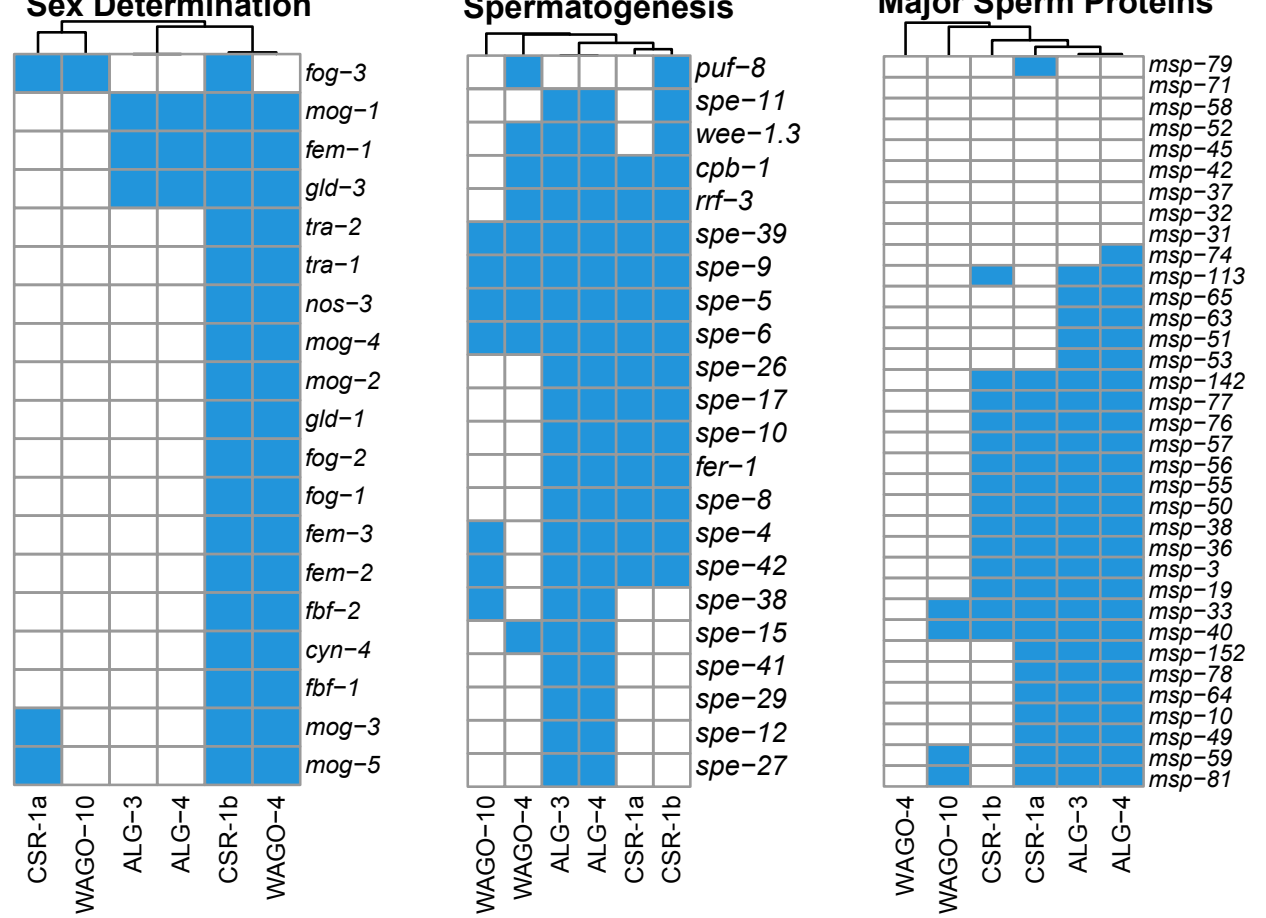

B

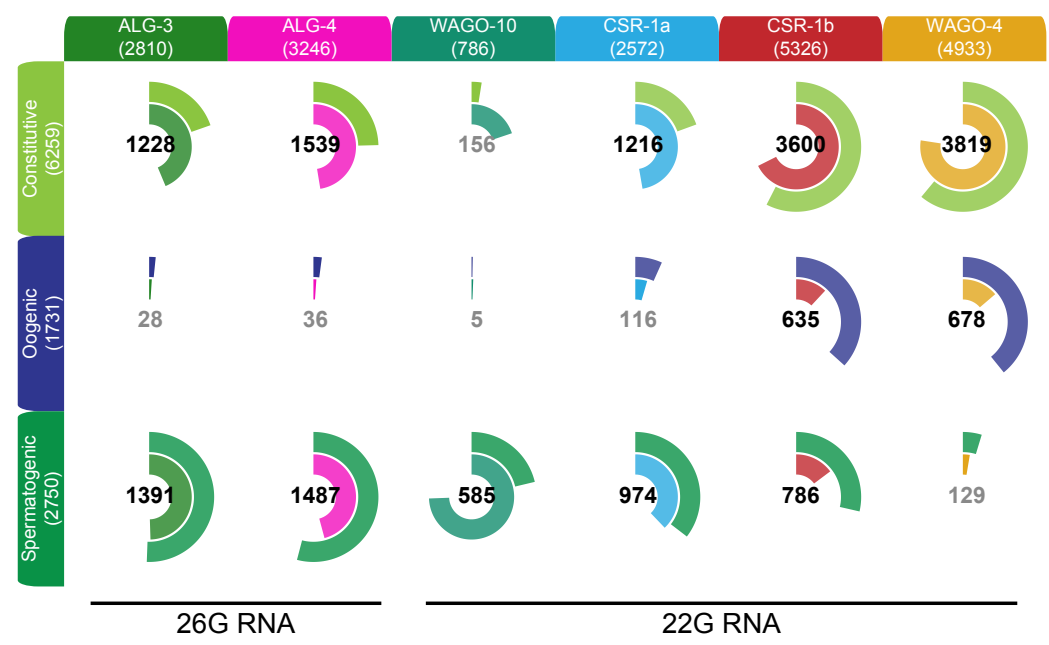

D Stem Cell Niche

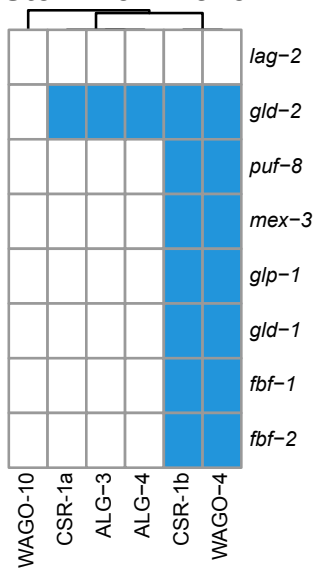

$\mathrm{H}$

Sperm Activation

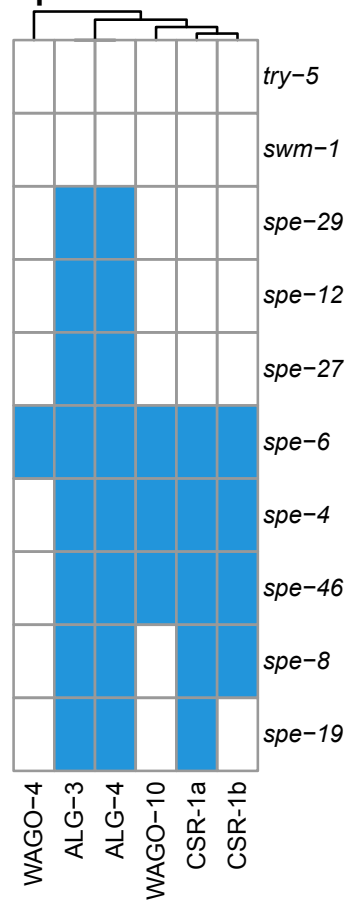


bioRxiv preprint doi: https://doi.org/10.1101/2020.07.20.212050; this version posted June 16, 2021. The copyright holder for this preprint (which was not certified by peer review) is the author/funder. All rights reserved. No reuse allowed without permission.

Figure 11. Model for differential functions of CSR-1a and CSR-1b

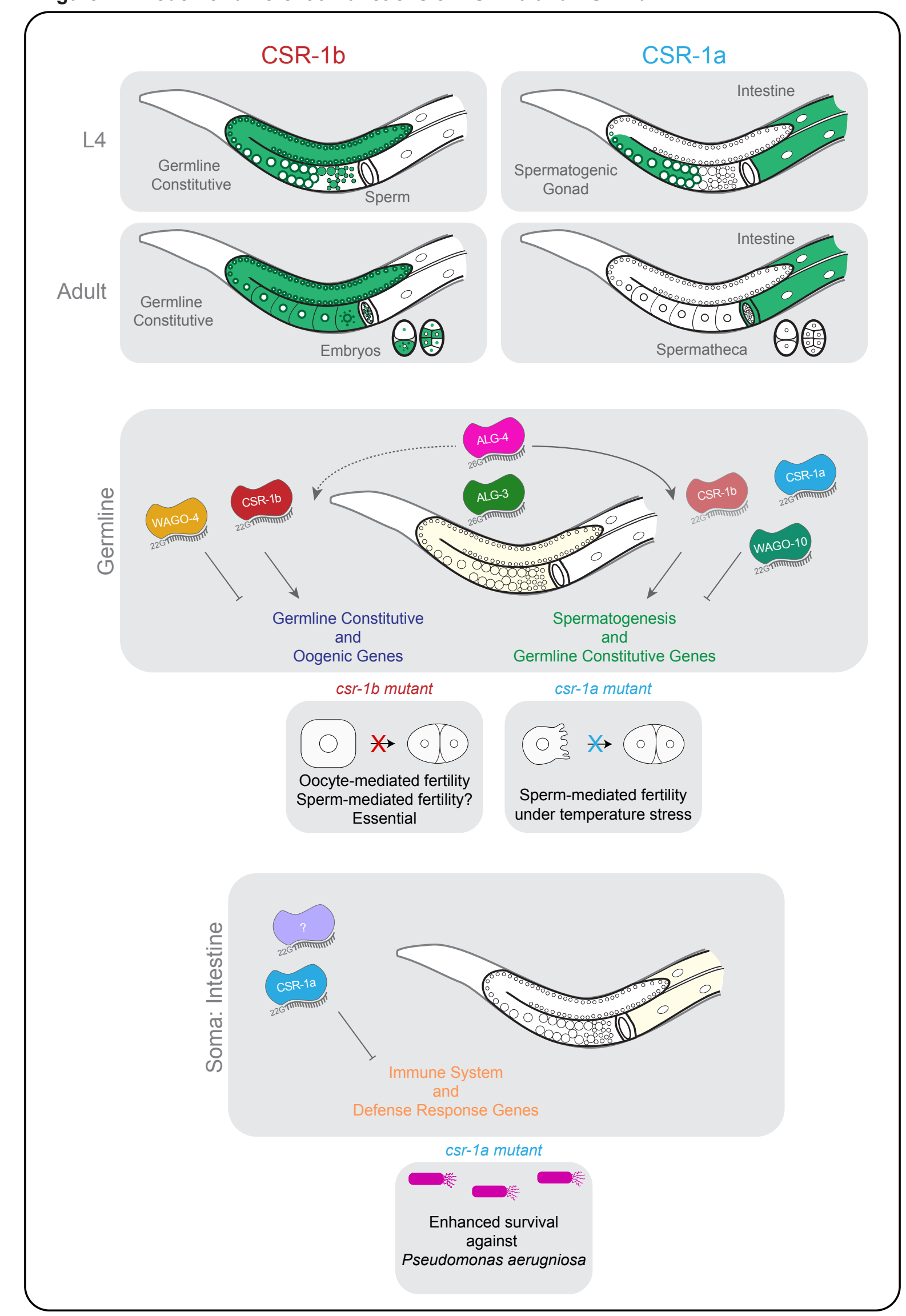

